\title{
Serotonin and cognitive flexibility : neuroimaging studies into the effect of acute tryptophan depletion in healthy volunteers
}

Citation for published version (APA):

Evers, E. A. T. (2006). Serotonin and cognitive flexibility : neuroimaging studies into the effect of acute tryptophan depletion in healthy volunteers. [Doctoral Thesis, Maastricht University]. NeuroPsych Publishers. https://doi.org/10.26481/dis.20060928ee

Document status and date:

Published: 01/01/2006

DOI:

10.26481/dis.20060928ee

Document Version:

Publisher's PDF, also known as Version of record

Please check the document version of this publication:

- A submitted manuscript is the version of the article upon submission and before peer-review. There can be important differences between the submitted version and the official published version of record.

People interested in the research are advised to contact the author for the final version of the publication, or visit the DOI to the publisher's website.

- The final author version and the galley proof are versions of the publication after peer review.

- The final published version features the final layout of the paper including the volume, issue and page numbers.

Link to publication

\footnotetext{
General rights rights.

- You may freely distribute the URL identifying the publication in the public portal. please follow below link for the End User Agreement:

www.umlib.nl/taverne-license

Take down policy

If you believe that this document breaches copyright please contact us at:

repository@maastrichtuniversity.nl

providing details and we will investigate your claim.
}

Copyright and moral rights for the publications made accessible in the public portal are retained by the authors and/or other copyright owners and it is a condition of accessing publications that users recognise and abide by the legal requirements associated with these

- Users may download and print one copy of any publication from the public portal for the purpose of private study or research.

- You may not further distribute the material or use it for any profit-making activity or commercial gain

If the publication is distributed under the terms of Article $25 \mathrm{fa}$ of the Dutch Copyright Act, indicated by the "Taverne" license above, 


\section{Serotonin and cognitive flexibility}

Neuroimaging studies into the effect of acute tryptophan depletion in healthy volunteers 
Printed by Datawyse BV, Maastricht

ISBN-10: 90-75579-28-4

ISBN-13: 978-90-75579-28-4

\section{(C) 2006 EAT Evers, Maastricht}

All rights are preserved, whether the whole or part is concerned. No part of this publication may be reproduced, stored in a retrieval system, or transmitted in any form or by any means, electronic, mechanical, photocopying, recording, or otherwise, without written permission from the copyright owner.

Neuropsych Publishers is a non-profit organization, which aims at promoting the science of 'Brain and Behavior' and improving the application of the products of this science in health care and education. Neuropsych Publishers accomplishes these aims by publishing books, dissertations and other products of scientific activity, by disseminating educational material and publication of tests, assessment scales and other psychometric instruments in the field of Neuropsychology, Neuropsychiatry and other areas within the domain of Brain and Behavior.

Postal address: Neuropsych Publishers, Department of Psychiatry and Neuropsychology, Maastricht University, P.O. Box 616, NL-6200 MD Maastricht, The Netherlands, wwwnp.unimaas.nl . 


\title{
Serotonin and cognitive flexibility
}

\section{Neuroimaging studies into the effect of acute tryptophan depletion in healthy volunteers}

\author{
Proefschrift \\ Ter verkrijging van de graad van doctor \\ aan de Universiteit Maastricht, \\ op gezag van Rector Magnificus, \\ Prof. mr. G. P. M. F. Mols \\ volgens het besluit van het College van Decanen, \\ in het openbaar te verdedigen \\ op donderdag 28 september 2006 om 14:00 uur
}

door

Elisabeth Anna Theresia Evers 


\section{Promotor}

Prof. dr. J. Jolles

\section{Copromotor}

Dr. F. M. van der Veen (Erasmus MC, Rotterdam)

\section{Beoordelingscommissie}

Prof. dr. W. J. Riedel (voorzitter)

Prof. dr. R. Goebel

Prof. dr. H. W. M. Steinbusch

Prof. dr. H. B. M. Uylings (Vrije Universiteit Amsterdam)

Prof. dr. A. J. W. Van der Does (Universiteit Leiden)

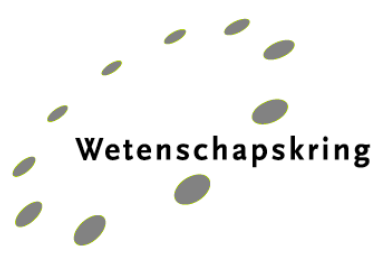

Mondriaan Zorggroep • Vijverdal • RIAGC Maastricht

- RGC Westelijke Mijnstreek - RIBW Heuvelland en Maasvallei

- Academisch Ziekenhuis Maastricht • Atrium Medisch Centrum

- Universiteit Maastricht • Onderzoeksschool Experimentele Psychopathologie

- Onderzoeksschool Hersenen en Gedrag

The research described in this thesis was performed at the Maastricht Brain and Behavior Institute, Department of Psychiatry and Neuropsychology, Maastricht University, Maastricht, The Netherlands and at the Department of Experimental Psychology, University of Cambridge, Cambridge, UK.

This work was supported by a TOP grant (No. 912-02-050) from ZonMWNWO.

Financial support for the publication of this thesis has been kindly provided by Pfizer BV, Wyeth Pharmaceuticals BV, AstraZeneca BV. 


\section{Contents}

Introduction

Chapter 1

Effects of a novel method of acute tryptophan depletion on plasma

tryptophan and cognitive performance in healthy volunteers

Chapter 2

Serotonergic modulation of prefrontal cortex during negative

feedback in probabilistic reversal learning

Chapter 3

The effect of acute tryptophan depletion on the BOLD response during performance monitoring and response inhibition in healthy male volunteers

\section{Chapter 4}

Acute tryptophan depletion improves performance and modulates the BOLD response during a Stroop task in healthy females

\section{Chapter 5}

A genetic predisposition to depression interacts with the effect of acute tryptophan depletion during a Stroop task

Chapter 6

The effect of tryptophan depletion on the error-related negativity

Concluding Remarks

References

Summary

Samenvatting

Curriculum vitae 



\section{Introduction}

Cognitive flexibility refers to the ability to switch between behavioral strategies, when changes in the environment lead to alterations in the outcome of actions. When cognitive flexibility is disturbed behavior becomes rigid. Previous research suggests that serotonin (5-HT) is involved in cognitive flexibility. Animal research showed that low 5-HT leads to inflexible behavior during reversal learning and response inhibition (Mazer et al., 1997; Barnes et al., 1990; Harrison et al., 1997; 1999). Clinical disorders that show abnormal 5-HT functioning, such as major depression and obsessive compulsive disorder (OCD), are associated with cognitive inflexibility (Veale et al., 1996; Houston et al., 2004; Fossati et al., 2002). Acute tryptophan depletion (ATD) is a well-established method to temporarily lower central 5-HT. ATD impaired the ability to perform a reversal shift in an ID/ED task (Rogers et al., 1999a; Park et al., 1994), impaired performance on a probabilistic reversal learning task (Murphy et al., 2002) and impaired decision making (Rogers et al., 2003) in healthy volunteers.

Although previous research suggests that 5-HT is important for cognitive flexibility, it is unknown how disturbed 5-HT levels change brain activation during tasks that require flexible behavior. Functional Magnetic Resonance Imaging (fMRI) is a neuroimaging technique that measures changes in blood oxygen levels. FMRI is used to investigate which brain areas are activated or deactivated during task performance. Neuroimaging studies suggest that especially the orbital and the dorsomedial part of the prefrontal cortex are important for cognitive flexibility (Bechara et al., 2000; O'Doherty et al., 2001). Importantly, fMRI will enable us to gain more insight into the effect of disturbed 5-HT functioning on brain activation associated with task performance. Thus far these brain mechanisms have not been studied. Because of the importance for treatment and prevention of disorders associated with rigid behavior, the goal of the present thesis is to gain more insight into the role of the PFC and 5-HT in cognitive flexibility. Before the aim and the approach of this thesis are presented, the following topics will be introduced: cognitive flexibility, 5-HT and the method of ATD.

\section{Cognitive flexibility and the prefrontal cortex}

In daily life cognitive flexibility is an important aspect of cognitive functioning that allows us to adjust behavior to a constantly changing environment. Disorders 
that are associated with cognitive inflexibility are for example OCD and affective disorders (Fossati et al., 2002; Houston et al., 2004). In experimental settings, cognitive flexibility refers to the ability to adjust responses to changes in reinforcement or task requirements. Cognitive flexibility is a multi component process, including performance monitoring, learning new stimulus-reward associations, behavioral adaptation and inhibition of the response related to the previous stimulus-reward association. Tasks that have been used to measure cognitive flexibility include reversal learning, decision making and gambling tasks. Typically, these tasks require the subjects to choose the appropriate response, and adjust the response strategy based on the feedback or cues they receive (correct/incorrect, reward/punishment, probabilities of reward/punishment) (e.g. O'Doherty et al., 2001; Elliott and Dolan, 1999; Manes et al., 2002). In a reversal learning task, for example, the participants have to decide on the basis of feedback whether the response rule has changed or not and adjust their behavior accordingly.

The prefrontal cortex (PFC) is important for higher cognitive functions, such as cognitive flexibility. Previous studies showed that lesions in the PFC can lead to more rigid behavior, such as impaired associative learning (Petrides 1982) and poor response inhibition (Milner 1964). On basis of the division of the mediodorsal nucleus of the thalamus, which projects to the PFC, the PFC can be divided into three general regions 1) the dorsolateral prefrontal cortex, 2) the orbitofrontal cortex, and 3) the frontal eye fields (Fuster, 1997). However, other divisions are possible.

The availability of neuroimaging techniques, such as fMRI has increased our insight into the role of the PFC in higher cognitive functions. FMRI measures the blood oxygen level dependent (BOLD) response, a measure for regional activation of the brain, while participants perform a cognitive task. Event-related fMRI is able to relate different cognitive events within one task, for example response inhibition and error processing in a Go/NoGo task, to specific brain activation patterns. Neuroimaging studies showed that higher cognitive functions rely on a widely distributed network of specialized anterior as well as posterior brain regions, of which the PFC consistently forms a part. Previous studies showed that the PFC is involved in cognitive flexibility. For example, Cools et al. (2002) showed that a behavioral switch on a reversal learning task increased the activation in the ventrolateral PFC, the dorsomedial PFC and the right parietal cortex, compared to correct responses. O'Doherty et al. (2001) showed that distinct regions of the orbitofrontal cortex were activated by rewards and punishment during a reversal learning task. 


\section{Serotonin and cognition}

One of the neurotransmitters that is thought to be involved in cognitive flexibility is serotonin (5-hydroxytryptamine; 5-HT). 5-HT is built from the essential amino acid tryptophan (TRP). TRP is synthesized by TRP hydroxylase to 5-hydroxytryptophan (5-HTP). 5-HTP is then synthesized by 5-HTP decarboxylase to 5-HT. To reach the brain, TRP must share the transport through the blood-brain barrier with other large neutral amino acids. Therefore not only plasma TRP levels but also the levels of the other large neutral amino acids are important for the availability of TRP for 5-HT synthesis. 5-HT projections originate from the midline raphe nucleus, and spread throughout the entire brain. 5-HT reaches the forebrain via the medial forebrain bundle and the dorsal raphe cortical tract (Baumgarten and Grozdanovic, 1997).

Acute tryptophan depletion (ATD) is a well-recognized research method for reducing central 5-HT in humans and studying the effects of low 5-HT on cognition. TRP is depleted by ingesting an amino acid (AA) mixture that does not contain TRP but does include other large neutral amino acids (Young et al., 1985). ATD is achieved by increasing protein synthesis in the liver with subsequent decreases in plasma TRP stores. In addition, the AA load results in more competition for the active transport system that the AAs share for entry across the blood-brain barrier, resulting in reduced availability of TRP in the brain. Previous studies showed that ATD successfully reduced the central 5-HT turnover in humans (Carpenter et al., 1998; Moreno et al., 2000; Nishizawa et al., 1997; Williams et al., 1999; Young et al., 1985, 1999). In a new method of ATD a lowTRP collagen-protein (CP) mixture is used (Lieben et al., 2004). This protein is derived from the selective hydrolysis of $\mathrm{CP}$ and comprises the entire range of AAs in the form of peptides. After administration, these peptides are decomposed into AAs, and the mechanism of depletion is identical to that of the AA mixture. This mixture proved successful in animals (Lieben et al., 2004), but has not yet been tested in humans.

ATD has not only been used to study the effect of a transient lowering of central 5-HT on cognition in healthy volunteers, it has also been used to study individual vulnerability of the 5-HT system. Previous studies showed that risk factors for developing depression (e.g. previous depressive episodes and a positive family history of affective disorder) trigger a mood response after ATD (Benkelfat et al., 1994; Booij et al., 2002; Ellenbogen et al., 1996; Klaassen et al., 1999; Riedel et al., 2002, 2003; Sobczak et al., 2002). Only few studies investigated the association between ATD, mood and cognitive performance in healthy volunteers with a genetic susceptibility to depression (Klaassen et al., 2002; Booij et al., 
2005; Munafo et al., 2006). It remains unclear, whether ATD affects cognition via individual mood changes, or via an independent effect on mood and cognition. No neuroimaging studies have been conducted to study the brain mechanisms related to the interaction between a genetic vulnerability to depression and the effect of ATD on cognition.

\section{The aim of this thesis}

In the preceding paragraphs we showed that cognitive flexibility is associated with the PFC and that especially the orbital and the dorsomedial PFC are important. In addition, we showed that previous studies suggested that 5-HT is implicated in cognitive flexibility. Disturbed 5-HT functioning in animals, patients and healthy volunteers leads to more rigid behavior. However, the brain mechanisms that underlie the effect of disturbed 5-HT functioning on cognitive flexibility are unknown. The main objective of this thesis is to gain more insight into the role of the PFC and 5-HT in cognitive flexibility in healthy volunteers.

\section{Approach}

\section{Acute tryptophan depletion}

The method of ATD was used in the present thesis to temporarily lower central 5-HT. In the first study a CP mixture (100 g +/- $1.2 \mathrm{~g}$ TRP) was used, in the other studies an AA mixture (75 g +/- $2 \mathrm{~g}$ TRP). Participants consumed the mixture when they arrived at the laboratory and were tested after a waiting period of 4 till 5 hours. Blood samples were taken to assess plasma AA levels. Mood and physical complaints questionnaires were completed to evaluate possible confounding effects.

\section{Participants}

For the present thesis male and female healthy volunteers were tested (mean age around 23). The participants did not suffer from present physical or psychological illnesses, had not been seriously ill in the past and did not use medication other than an oral anti-conceptive. In addition, the participants had never used XTC or antidepressants. In chapters 4 and 5, healthy females with $(\mathrm{FH}+)$ and without $(\mathrm{FH}-)$ a positive family history of unipolar depression were included. 


\section{Neuroimaging}

In study 2 till 5 event-related fMRI was used to study the effects of ATD on the BOLD response. Because of the superior temporal resolution of electroencephalography (EEG) as compared to fMRI, the last study used EEG to examine the effect of ATD on the error-related brain response.

\section{Cognition}

In the present thesis reversal learning was used to measure cognitive flexibility (Chapter 1 and 2). In addition, other tasks were used to examine sub processes that are important for cognitive flexibility, such as response inhibition (Go/NoGo task; Chapter 3), cognitive control during response interference (Stroop task; Chapter 4 and 5) and performance monitoring (Go/NoGo and the Eriksen Flanker task; Chapter 3 and 6).

\section{Outline of this thesis}

In Chapter 1 we examined the effect of a novel method of ATD (a natural CP mixture) on cognition. Fifteen healthy volunteers were tested in a counterbalanced placebo-controlled within-subject design. Reversal learning, verbal memory and pattern recognition were assessed at baseline and 3-4 h after taking the $\mathrm{CP}$ mixture.

In Chapter 2 we investigated the effect of a transient lowering of 5-HT on brain activation associated with cognitive flexibility. An event-related fMRI study is described that examined the effect of ATD on task performance and the BOLD response during a probabilistic reversal learning task. Twelve healthy male volunteers were tested in a counterbalanced placebo-controlled within-subject design.

In Chapter 3 we examined the effect of a transient lowering of 5-HT on brain activation associated with two sub processes sub serving cognitive flexibility, namely response inhibition and task performance monitoring. An event-related fMRI study is described that examined the effect of ATD on performance and the BOLD response during a modified $\mathrm{Go} / \mathrm{NoGo}$ task. Thirteen healthy male volunteers were tested in a counterbalanced placebo-controlled within-subject design.

In Chapter 4 we examined the effect of a transient lowering of 5-HT on brain activation associated with cognitive control, which is needed during response interference from distracting task irrelevant stimuli. An event-related fMRI study 
is described that examined the effect of ATD on task performance and the BOLD response during a combined cognitive and emotional Stroop task. Fifteen healthy female volunteers were tested in a counterbalanced placebo-controlled withinsubject design.

In Chapter 5 we studied the interaction between a genetic predisposition to depression and the effect of a transient lowering of 5-HT on mood and brain activation during cognitive control. An event-related fMRI study is described that examined the effect of ATD on mood, cognitive performance and the BOLD response during a combined emotional and cognitive Stroop task. Twelve healthy females with a positive family history of unipolar depression were tested in a counterbalanced placebo-controlled within-subject design. They were compared with fifteen healthy females (participants from chapter 4) without a family history of depression.

In Chapter 6 we studied the effect of a transient lowering of 5-HT on brain activation related to a response error. An EEG study is described that examined the effect of ATD on mood, cognitive performance and the error-related negativity (ERN) during an Eriksen Flanker task. Twenty healthy male volunteers were tested in a counterbalanced placebo-controlled within-subject design.

Finally, concluding remarks are presented. 


\title{
Chapter 1
}

\section{Effects of a novel method of acute tryptophan depletion on plasma tryptophan and cognitive performance in healthy volunteers}

EAT Evers, DE Tillie, FM van der Veen, CK Lieben, J Jolles, NEP Deutz, JAJ Schmitt

\begin{abstract}
Rationale. Disorders associated with low levels of serotonin (5-HT) are characterized by mood and cognitive disturbances. Acute tryptophan depletion (ATD) is an established method for lowering 5-HT levels and an important tool to study the effects of reduced 5-HT on mood and cognition in human subjects. The traditional ATD method, i.e., administration of separate amino acids (AAs), has several disadvantages. The AA mixture is costly, unpalatable and associated with gastrointestinal discomfort. Objectives. The University of Maastricht developed a new and inexpensive method for ATD: a natural collagen protein (CP) mixture with low tryptophan (TRP) content. The reductions in plasma TRP after taking this CP mixture were compared with the reductions achieved taking the traditional AA mixture, and effects on memory and reversal learning were studied. Methods. Fifteen healthy young volunteers participated in a double-blind, counterbalanced within-subject study. Reversal learning, verbal memory and pattern recognition were assessed at baseline and 3-4 h after taking the $\mathrm{CP}$ mixture. Results. The new ATD method significantly reduced plasma TRP by $74 \%$ and the ratio between TRP and the other large AAs (TRP/ $\Sigma$ LNAA) by $82 \%$. The placebo mixture did not change these measures. Delayed recognition reaction time on the verbal learning task was increased following ATD. No other cognitive effects were found. Conclusions. The CP mixture was shown to be an efficient tool for lowering plasma TRP in humans. The validity of this method with regard to behavioral changes remains to be established in healthy, vulnerable and clinical populations.
\end{abstract}

\section{Acknowledgements}

This work was supported by a TOP grant (No. 912-02-050) from ZonMW-NWO.

Published in Psychopharmacology (Berl). 2005 Feb;178(1):92-9. 


\section{Introduction}

Serotonergic (5-HT) dysfunction is associated with disrupted cognitive function, emotional processing and social functioning, which are characteristic for depressive patients (Murphy et al., 2002). A pharmacological model to study the role of 5-HT in human behavior is acute tryptophan depletion (ATD), in which central 5-HT synthesis is reduced by lowering the brain availability of the 5-HT precursor L-tryptophan (TRP) (reviewed by Reilly et al., 1997). Animal (Biggio et al., 1974; Gartside et al., 1992) and human (Nishizawa et al., 1997, Carpenter et al., 1998; Williams et al., 1999) studies have demonstrated that the acute reduction of TRP to the brain is sufficient to produce a rapid decrease in the synthesis and release of brain $5-\mathrm{HT}$.

Previous ATD studies showed impaired performance on tasks involving memory consolidation (see Riedel et al., 2002 for an overview), decision making (Rogers et al., 1999b, 2003), reversal learning (Murphy et al., 2002; Rogers et al., 1999a) and affective processing (Murphy et al., 2002; Rubinzstein et al., 2001) in healthy volunteers. The aim of the present study was to investigate the effects of a novel ATD method on the plasma TRP levels, on the ratio between TRP and the other large neutral amino acids ( $L$ LNAAs), and to validate the method on a behavioral level. To this end, we assessed several cognitive functions that are known to be sensitive to ATD, i.e., memory and reversal learning.

A low-TRP collagen-protein (CP) mixture was used to induce an ATD. This protein is derived from the selective hydrolysis of $\mathrm{CP}$ and comprises the entire range of amino acids (AAs) in the form of peptides. After administration, these peptides are decomposed into AAs, and the mechanism of depletion is identical to that of the AA mixture. In rats, the CP mixture significantly lowered plasma TRP and TRP/ $/$ LNAA ratios $(-78 \%)$ and brain TRP and 5-HT (-50\%) concentrations (Lieben et al., 2004). Based on the previous behavioral ATD studies and the demonstration that the CP mixture was an efficient TRP-depletion method in rats, it was hypothesized that this new method of ATD results in reduced plasma TRP, a decreased TRP/ $\Sigma$ LNAA ratio, impaired delayed recall and recognition in word and pattern-learning tasks, slower responding and more errors in the reversallearning task when compared with the placebo in healthy volunteers. 


\section{Materials and methods}

\section{Subjects}

Fifteen young healthy male $(n=3)$ and female $(n=12)$ volunteers (mean age 21.8 years; $\mathrm{SD}=1.8$ ) gave informed consent and participated in this study, which was approved by the medical ethics committee of the University Hospital, Maastricht. The participants were free from significant past or present physical or psychiatric illness and did not use medication other than oral anti-conceptives. Female participants were not tested in the late luteal phase of the menstrual cycle (days 21-28). All subjects completed the study.

\section{CP mixture}

The CP mixture was purchased from PB Gelatins (Tessenderlo, Belgium); see Table 1 for the AA composition. To obtain a drinkable mixture, $100 \mathrm{~g}$ of the CP mixture was mixed with $200 \mathrm{~g}$ water. The placebo mixture was identical in composition, but 1.2 g L-TRP (Sigma, Zwijndrecht, The Netherlands) was added.

\section{Design}

This study was conducted according to a double-blind, placebo-controlled crossover design. The participants received a TRP-free CP mixture $\left(\mathrm{TRP}^{-}\right)$and a CP mixture with $1.21 \mathrm{~g}$ TRP added (placebo) on separate occasions. Treatment order was balanced over the two test days, which were separated by at least 3 days.

Table 1. Composition (grams) of the natural collagen protein (tryptophan ${ }^{-}$) in $100 \mathrm{ml}$ tap water

\begin{tabular}{lcll}
\hline Aspartic acid + asparagines & 5.2 & Tyrosine & 0.4 \\
Glutamic acid + glutamine & 9.3 & Valine & 2.1 \\
Hydroxyproline & 12.1 & Methionine & 0.6 \\
Serine & 3.1 & Cysteine & 0.2 \\
Glycine & 22.5 & Isoleucine & 1.4 \\
Histidine & 0.5 & Leucine & 3.0 \\
Arginine & 8.8 & Hydroxylysine & 1.4 \\
Threonine & 1.1 & Phenylalanine & 1.9 \\
Alanine & 9.3 & Tryptophan & 0.1 \\
Proline & 13.3 & Lysine & 3.6 \\
\hline
\end{tabular}




\section{Procedure}

In a separate session, approximately 1 week before the actual test days, the cognitive tasks were practiced to minimize learning effects. The participants were instructed not to drink alcohol on the days prior to the test days, not to eat or drink (except water) after 2200 hours that evening and to arrive at the laboratory well rested. A test day started with a cognitive test battery, subjective assessments of mood and adverse effects, and baseline blood sampling. Subsequently, subjects received the $\mathrm{TRP}^{-}$or the placebo mixture. A 3-h break followed to maximize TRP depletion. The participants had free access to low-TRP food, such as apples, tomatoes and protein-free candy, and caffeine-free tea during the pause. These food items were generally consumed in small quantities, and the intake of carbohydrates does not meaningfully affect brain TRP availability in the presence of large amounts of protein (Teff et al., 1989). After the 3-h interval, cognition, mood and adverse effects were assessed again and a blood sample was taken. The duration of a test day was $5 \mathrm{~h}$ in total.

\section{Cognitive assessment}

The cognitive test battery, mood and adverse effects assessments took approximately $1 \mathrm{~h}$ to complete. On each session parallel test versions were used, version order was distributed among the participants using a $4 \times 4$ Latin square.

\section{Probabilistic reversal learning task}

In the reversal-learning task (described in detail by O' Doherty et al., 2001), two abstract stimuli, composed of two bars of different color, were randomly presented to the left or right side of a computer screen. One stimuli was advantageous $(\mathrm{S}+)$ and was usually $(70 \%)$ associated with large reward (addition of 80-250 points) and occasionally (30\%) with a small punishment (subtraction of 10-60 points), based on a pseudo-random sequence. The other stimulus was disadvantageous (S-) and usually (60\%) associated with a large punishment (250600 points) and occasionally (40\%) a small reward (30-60 points). Volunteers had to determine which stimulus was advantageous based on the feedback-the number of points won or lost. Once this was learned, i.e., the advantageous stimulus was chosen four times out of five responses, the stimulus-reward contingencies were reversed $(\mathrm{S}+$ became $\mathrm{S}-$, and $\mathrm{S}-$ became $\mathrm{S}+$ ). The participant $\mathrm{s}$ task was to keep track of the most profitable stimulus and to collect as many points as possible. Two successive 8-min blocks were performed per session. The stimuli used in the two reversal blocks were four differently colored abstract patterns, and blocks were randomly assigned. The task stopped after the 19th reversal or after 121 trials. Before each assessment, subjects practiced an acquisition (eight correct responses in a row) and a short reversal-learning task (two reversals within 50 trials: 8 correct out of 9 trials). Dependent variables were 
the total number of reversals, the total number of perseverations (number of errors directly after the rule has changed), the mean reaction time per trial, the mean reaction time of the first response following a reversal switch and the total number of points collected, in the two successive reversal blocks.

\section{The visual verbal learning test}

The visual verbal learning test (VVLT), a modified version of the Rey Auditory Verbal Learning Test (Lezak 1995), consisted of a list of 30 monosyllabic words in Dutch, which were presented in three trials on a computer screen (Riedel et al., 1999). Of the words, 12 were positively loaded, 12 were negatively loaded and 6 were neutrally loaded. Immediately after each presentation (immediate recall) and 30 min later (delayed recall), the subjects were asked what words they could remember. After that, a delayed recognition test was presented; 30 words were shown (15 new and 15 words from the original word list), and the subject was asked to press "yes" for familiar and "no" for new, as quickly as possible. According to the theory of signal detection (Pollack and Norman, 1964), the proportion of correctly recognized words (cr) and the proportion of those falsely recognized (fr) constituted the non-parametric sensitivity measure: $A^{\prime}=1-1 / 4(\mathrm{fr} / \mathrm{cr}+(1-\mathrm{cr}) /(1-\mathrm{fr}))$, where $\mathrm{A}$ is in fact the proportion of correctly recognized words corrected for the participant's response tendency. A was arc sin transformed before statistical analyses. The outcome variables were the number of correct words recalled during the three immediate recall trials as a measure of short-term memory, the number of correct words produced on delayed free recall as a measure of retrieval from long-term memory, $A^{\prime}$ as a measure of storage in long-term memory, and the medial reaction times of correctly recognized words as a measure of speed of long-term memory.

\section{Abstract pattern learning task}

In the abstract pattern-learning task, 15 abstract stimuli were shown one by one on a computer screen, followed by the recognition task in which the participants had to press the left button of a response box if they recognized the left stimulus and the right button if they recognized the right stimulus. Recognition was tested immediately after the presentation of the patterns (immediate recognition) and 30 min later (delayed recognition). The dependent measure of interest was the proportion correctly recognized patterns and the reaction times of the correct responses.

\section{Questionnaires \\ Mood}

A visual analogue version of the profile of mood states (POMS) was used to assess mood (McNair et al., 1988). This questionnaire consists of 32 bipolar sets 
of adjectives, which measure five mood dimensions: anger, depression, fatigue, tension and vigor. The items were scored on a 0 - to $100-\mathrm{mm}$ scale. When the participants felt as they normally do, they were asked to mark the middle of the line (score 50).

\section{Adverse effects}

Adverse effects, 31 items, were registered and scored on a five-point scale from "no complaint at all" (0) to "severe complaint" (4).

\section{Plasma AAs}

In total, four blood samples $(4 \mathrm{ml})$ were drawn from each volunteer and immediately centrifuged at $4^{\circ} \mathrm{C}(10 \mathrm{~min}, 4500 \mathrm{rpm})$. A $100-\mu 1$ aliquot of plasma was mixed with $8 \mathrm{mg}$ sulfasalicyl acid and frozen at $-80^{\circ} \mathrm{C}$ until AA analysis using high-performance liquid chromatography (van Eijk 1993).

\section{Statistical analysis}

The outcome variables of the cognitive and mood assessments, and the total number of adverse effects were analyzed using a repeated-measures analysis of variance (ANOVA) of the difference scores (performance scores 3-4 h after the drink administration minus baseline scores) using treatment (TRP ${ }^{-}$, placebo) as within-subject factor and treatment order $\left(\mathrm{TRP}^{-}\right.$or placebo mixture first) as between-subject factors.

\section{Results}

\section{Levels of plasma TRP}

Blood samples of two participants were missing. Figure 1 shows that plasma TRP concentrations and TRP/ $\Sigma$ LNAA ratios at baseline did not differ between groups. Three hours after drinking the $\mathrm{TRP}^{-}$mixture, plasma TRP was significantly reduced by $74 \%\left(F_{1,11}=60.8, P<0.001\right)$ and the TRP/ $\Sigma$ LNAA ratios by $82 \% \quad\left(F_{1,11}=50.4, P<0.001\right)$, compared with baseline. Small, non-significant changes in plasma TRP $(+8 \%)$ and TRP/ $/$ LNAA ratio $(-2 \%)$ were observed following administration of the placebo mixture.

\section{Cognitive assessments}

The results of the cognitive assessments are summarized in Table 2. Delayed recognition reaction time of the VVLT was increased following ATD $\left(F_{1,13}=9.2\right.$, $P=0.01$ ). No treatment effects were seen on delayed recognition accuracy, delayed recall or overall immediate recall. No effects of ATD were seen on the outcome variables of the probabilistic reversal-learning task and the abstract pattern- 
learning task. None of the analyses showed an interaction between treatment order and the effects of the treatment.

\section{Adverse effects and mood assessment}

None of the subscales of the POMS - depression, anger, tension, fatigue and vigor-showed an effect of treatment (Table 3). As is shown in Table 4, neither the $\mathrm{TRP}^{-}$nor the placebo mixture was associated with robust adverse effects at $\mathrm{T} 4$. None of the participants scored above " 1 " (bothered a bit) on any of the adverse effect items. The total adverse-effects score was identical in the ATD versus the placebo condition. Treatment order did not modify the outcome of the analyses.
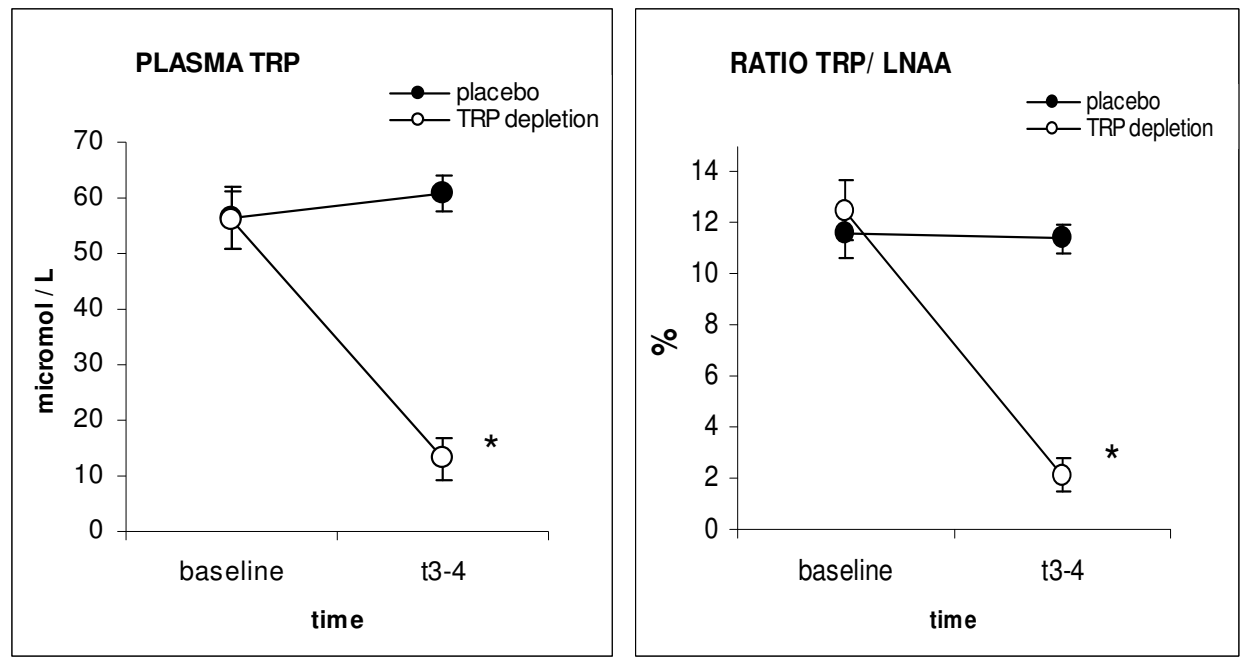

Figure 1. The means and the standard errors (SE) of the blood plasma levels of tryptophan $(\mu \mathrm{mol} / \mathrm{l})$ and the ratio between tryptophan and the other large amino acids (TRP/LLNAA) at baseline and $4 \mathrm{~h}$ after start of the TRP depletion and placebo treatment. $* P<0.001$ 
Table 2. Mean scores (standard deviations) for the outcome variables of the visual verbal learning task, the probabilistic reversal learning task and the abstract visual learning task at baseline and 3-4 $\mathrm{h}$ after administration of the tryptophan (TRP) ${ }^{-}$and placebo mixture.

\begin{tabular}{|c|c|c|c|c|}
\hline & \multicolumn{2}{|c|}{$\mathrm{TRP}^{-}$condition } & \multicolumn{2}{|c|}{ Placebo condition } \\
\hline & Baseline & T3-4 & Baseline & T3-4 \\
\hline \multicolumn{5}{|l|}{ Visual verbal learning task } \\
\hline \multicolumn{5}{|c|}{ Number of words correctly recalled on the three immediate recall trials } \\
\hline Trial 1 & $12.1(3.8)$ & $9.4(3.3)$ & $11.9(3.1)$ & $10.3(2.7)$ \\
\hline Trial 2 & $17.5(4.4)$ & $15.0(4.9)$ & $18.1(3.6)$ & $16.7(3.8)$ \\
\hline Trial 3 & $21.5(3.9)$ & $18.9(7.7)$ & $21.7(3.7)$ & $20.4(4.2)$ \\
\hline $\begin{array}{l}\text { Words correctly recalled on } \\
\text { delayed recall }\end{array}$ & $18.7(6.2)$ & $13.7(7.0)$ & $19.7(6.0)$ & $15.0(5.5)$ \\
\hline $\begin{array}{l}\text { Delayed recognition } \\
\text { sensitivity measure A }(\%)\end{array}$ & $96(4)$ & $94(6)$ & $97(2)$ & $95(6)$ \\
\hline $\begin{array}{l}\text { Median RT of correctly } \\
\text { recognized words (ms) }\end{array}$ & $677(92)$ & $703(98) *$ & 669 (69) & $654(74)$ \\
\hline \multicolumn{5}{|c|}{ Probabilistic reversal learning task } \\
\hline Total number of reversals & $18.0(1.5)$ & $18.3(1.3)$ & $18.1(1.1)$ & $18.6(1.1)$ \\
\hline $\begin{array}{l}\text { Total number of } \\
\text { perseverations }\end{array}$ & $26.0(7.9)$ & $25.1(6.9)$ & $25.5(6.6)$ & $23.1(6.7)$ \\
\hline Mean RT & $496(88)$ & 479 (109) & $464(89)$ & $426(64)$ \\
\hline Mean RT after a reversal & $487(90)$ & $474(114)$ & $461(94)$ & $436(76)$ \\
\hline Total number of points & $1381(1217)$ & $1451(1674)$ & $1400(1776)$ & $1732(2173)$ \\
\hline \multicolumn{5}{|c|}{ Abstract pattern recognition task } \\
\hline \multicolumn{5}{|c|}{ Proportion correctly recognized patterns } \\
\hline Immediate recognition & $90.6(11.0)$ & $89.6(14.0)$ & $92.6(6.2)$ & $93.1(8.1)$ \\
\hline Delayed recognition & $91.0(8.3)$ & $82.8(15.6)$ & $88.3(8.2)$ & $85.6(11.7)$ \\
\hline \multicolumn{5}{|c|}{ Reaction times for the correct responses } \\
\hline Immediate recognition & $1604(451)$ & $1613(498)$ & $1580(434)$ & $1593(436)$ \\
\hline Delayed recognition & 1593 (411) & $1758(486)$ & $1503(372)$ & $1597(530)$ \\
\hline
\end{tabular}
$* \mathrm{p}=0.01$ 
Table 3. Mean (standard deviation) scores on the subscales of the profile of mood questionnaire at baseline and $4 \mathrm{~h}$ after administration of the tryptophan (TRP)-depleted mixture (TRP ${ }^{-}$) and the placebo mixture

\begin{tabular}{llccc}
\hline & \multicolumn{2}{c}{ TRP $^{-}$condition } & \multicolumn{2}{c}{ Placebo } \\
& Baseline & T4 & Baseline & T4 \\
\hline Depression & $5.6(0.9)$ & $5.5(1.0)$ & $5.5(1.3)$ & $5.4(1.3)$ \\
Anger & $5.7(1.0)$ & $5.6(1.2)$ & $5.6(1.4)$ & $5.5(1.4)$ \\
Fatigue & $5.1(0.9)$ & $4.9(1.0)$ & $4.9(1.2)$ & $4.8(1.1)$ \\
Vigor & $4.7(0.8)$ & $5.3(1.0)$ & $6.0(3.4)$ & $5.2(0.9)$ \\
Tension & $5.4(1.0)$ & $5.4(0.8)$ & $5.6(1.4)$ & $5.6(1.3)$ \\
\hline
\end{tabular}

Table 5. The decrease (in percentages) in total plasma tryptophan (TRP), the ratio between TRP and the other large amino acids (ratio) in the tryptophan depletion $\left(\mathrm{TRP}^{-}\right)$and the placebo conditions as a result of acute TRP depletion (ATD) by amino acid administration and the collagen protein (CP) method (present study). The amount of amino acids (AA) and TRP in grams (g), and the number of hours $(t)$ between consuming the mixture and the providing blood samples are given.

\begin{tabular}{lccccccc}
\hline & \multicolumn{2}{c}{ Plasma TRP } & \multicolumn{2}{c}{ Ratio } & \multirow{2}{*}{ AA } & TRP & $\mathrm{t}$ \\
& TRP $^{-}$ & Placebo & TRP $^{-}$ & Placebo & & & \\
\hline Riedel et al. (1999) & -67 & +22 & -78 & -20 & 100 & 3.0 & 6 \\
Schmitt et al. (2000) & -63 & +80 & -79 & +20 & 100 & 4.6 & 5 \\
Rubinsztein et al. (2001) & -80 & +47 & & & 53 & 2.0 & 5 \\
Murphy et al. (2002) & -70 & & & 86 & 1.9 & 7 \\
Anderson et al. (2003) & -84 & +118 & & & $100 / 86^{\mathrm{a}}$ & $2.3 / 1.8^{\mathrm{a}}$ & 6 \\
Present study & -74 & +8 & -82 & -2 & 100 & 1.2 & 4 \\
\hline
\end{tabular}

${ }^{\mathrm{a}} 100 \mathrm{~g}$ of AAs with $2.3 \mathrm{~g}$ TRP added for males and $86 \mathrm{~g}$ of AAs with $1.8 \mathrm{~g}$ TRP added for females 
Table 4. The number of participants experiencing the physical complaint mentioned

\begin{tabular}{|c|c|c|c|c|}
\hline \multirow[b]{2}{*}{ Adverse effects } & \multicolumn{2}{|c|}{$\mathrm{TRP}^{-}$condition } & \multicolumn{2}{|c|}{ Placebo } \\
\hline & Baseline & $\mathrm{T} 4$ & Baseline & $\mathrm{T} 4$ \\
\hline Headache & 3 & 2 & 4 & 6 \\
\hline Sleepiness & 8 & 9 & 10 & 7 \\
\hline Dizziness & 0 & 0 & 3 & 3 \\
\hline Nausea & 1 & 4 & 1 & 4 \\
\hline Restlessness & 2 & 3 & 5 & 3 \\
\hline Heart palpitations & 0 & 0 & 0 & 0 \\
\hline Stomach ache & 1 & 1 & 1 & 2 \\
\hline Bloating & 2 & 5 & 0 & 5 \\
\hline Heartburn & 0 & 2 & 0 & 1 \\
\hline Loss of appetite & 0 & 5 & 1 & 5 \\
\hline Hunger & 12 & 5 & 13 & 6 \\
\hline Diarrhea & 0 & 1 & 0 & 0 \\
\hline Feeling cold & 7 & 5 & 7 & 5 \\
\hline Feeling warm & 1 & 1 & 0 & 0 \\
\hline Dry mouth & 6 & 2 & 7 & 6 \\
\hline Trembling & 0 & 0 & 2 & 2 \\
\hline Feeling tired & 10 & 10 & 9 & 10 \\
\hline A hazy view & 1 & 0 & 2 & 2 \\
\hline Sweating & 0 & 0 & 0 & 1 \\
\hline Sedation & 7 & 8 & 7 & 8 \\
\hline Feeling feeble & 5 & 8 & 4 & 4 \\
\hline Tightness in the chest & 1 & 0 & 0 & 0 \\
\hline Decreased concentration & 3 & 8 & 3 & 11 \\
\hline Tingling & 0 & 0 & 0 & 1 \\
\hline Nervousness & 2 & 0 & 3 & 0 \\
\hline Irritation & 1 & 1 & 0 & 0 \\
\hline Listless & 3 & 2 & 1 & 4 \\
\hline Bothered by bright light & 0 & 0 & 0 & 0 \\
\hline Bothered by hard sounds & 1 & 0 & 0 & 0 \\
\hline A warm head & 1 & 2 & 1 & 2 \\
\hline The feeling that you could faint & 1 & 1 & 0 & 0 \\
\hline
\end{tabular}

\section{Discussion}

The TRP ${ }^{-}$CP mixture appears to be an efficient tool to lower plasma TRP in humans. Four hours after administration, plasma TRP concentrations were reduced by $74 \%$ and the ratio TRP/ $\Sigma$ LNAA by $82 \%$. These levels of TRP depletion are comparable to those obtained by administration of the "classic" AA mixture (Table 5). Furthermore, the present results show that our placebo mixture is essentially neutral with regard to effects on plasma TRP as well as the TRP/LLNAA ratio. This constitutes an important advantage over placebo AA mixtures, in which neutral TRP/ $/$ LNAA ratios can only be maintained at the cost 
of marked increases of TRP levels (van der Does 2001; Weltzin et al., 1994). Both TRP/ $/$ LNAA (van der Does 2001; Moore et al., 2000) and free TRP levels (Biggio et al., 1974; Moja et al., 1989; Fadda 2000) have been suggested as being the predominant mediator of central TRP availability, meaning that changes in either parameter may result in an active control that may either underestimate or overestimate the effects of the depletion drinks (Reilly et al., 1997).

Although poorly documented, consumption of an ATD and placebo AA drink can be associated with quite unpleasant adverse effects, particularly gastrointestinal complaints with occasional vomiting, in some individuals. These adverse events occur predominantly in the first $1 \mathrm{~h}$ or $2 \mathrm{~h}$ after drink administration and subside in the ensuing hours, having little or no impact on assessments done $5 \mathrm{~h}$ or $6 \mathrm{~h}$ after the start of the treatment. However, these transient adverse effects can be quite discomforting for the subjects and may ultimately lead to subjects withdrawing from the study (Schmitt et al., 2000; Riedel et al., 1999; Danjou et al., 1990; Klaassen et al., 1999), while vomiting may diminish the level of depletion. It is clear that avoiding or minimizing adverse side effects would be highly desirable. In the present study, four subjects (all female) reported significant nausea, three of whom vomited, approximately $1 \mathrm{~h}$ after drink administration. Vomiting or nausea was not specifically related to either the TRP (two occasions) or placebo (two occasions) mixtures. Vomiting did not substantially affect the level of TRP depletion: plasma TRP level was reduced by $80 \%$ and $94 \%$, TRP/ $/ \Sigma$ LNAA ratios by $95 \%$ and $95 \%$ in the two individuals mentioned. Further, excluding these subjects from analyses of the cognitive data did not affect the outcome. Although the current study was not specifically designed to investigate the time course, magnitude and prevalence of drink-related side effects - this would require multiple assessments over time and a non-drink group to ascertain time of day effects-it was speculated that the CP mixture would elicit minimal gastrointestinal effects because of its increased palatability and lack of separate AAs. Our current observations do not support this notion, but more systematic investigation is warranted. However, our data do clearly show that at the time of testing, i.e., 3-4 h after drink administration, the $\mathrm{CP}$ mixtures are not associated with significant adverse effects, which could interfere with performance and mood assessments.

Administration of $\mathrm{TRP}^{-} \mathrm{CP}$ protein induced a mild reduction of long-term memory function, which was apparent only in terms of reduced speed of delayed word recognition, but not accuracy. It is important to note that slowing of responses in the word recognition task cannot be attributed to a general reduction of psychomotor speed as no effects of ATD were seen on reaction time measures of other tasks. Overall, the memory effect is rather modest compared with previous findings showing impaired accuracy of delayed recall and/or recognition 
(Harrison et al., 2004; Sobczak et al., 2002; Riedel et al., 1999; Schmitt et al., 2000), as well as reduced speed of recognition (Riedel et al., 1999). Nevertheless, our findings are in line with accumulating evidence implicating 5-HT in long-term memory functioning (Riedel 2004; Buhot et al., 2000; Meneses 1999). It is therefore rather unexpected that long-term memory for abstract patterns was unaffected by ATD in the current study. Our results conflict with those reported by Rubinzstein et al. (2001), who found that ATD impaired delayed recognition of previously presented abstract patterns. However, in the latter study, feedback was given on the correctness of each response, and task performance may have been modulated by altered feedback processing following ATD. Elliott et al. (1996) showed that depressed patients [associated with reduced levels of serotonin (Maes and Meltzer, 1995)] were oversensitive to negative feedback and in general showed a bias for negative stimuli (Murphy et al., 1999). The absence of ATD effects on this task, however, should also be viewed in the context of the overall picture of rather modest ATD effects, which may be related to more general methodological factors.

An overall explanation for the relatively mild long-term memory effects may be the timing of the post-treatment assessments. Generally, a 4-h to 5-h interval is maintained between ATD drink administration and subsequent testing, allowing for a maximal depletion of peripheral and presumably central TRP levels. A limited set of data from a series of pilot studies (unpublished data) suggested that maximal TRP depletion following $\mathrm{TRP}^{-} \mathrm{CP}$ administration was achieved after a 3$\mathrm{h}$ to 4-h interval (Fig. 2). However, it is possible that, due to a delay between peripheral depletion and central 5-HT deficiency (Biggio et al., 1974), this time may be too short to produce robust effects on memory function. In addition, it is possible that the use of an active control mixture that increases TRP levels leads to an overestimation of the cognitive effects of ATD in previous studies, since TRP depletion may have been compared with a state of enhanced TRP availability (Reilly et al., 1997). It can be argued that these factors may also underlie the absence of effect on the probabilistic reversal-learning task. However, other factors relating to the procedure and task characteristics may have also influenced the results. Task familiarity has been shown to modulate the effects of ATD on a number of tests of executive functioning (Gallagher et al., 2003), including probabilistic reversal learning (Murphy et al., 2002). It appears that ATD may diminish performance predominantly when the task is novel to the participants, e.g., on a single reversal switch on the first test day (Murphy et al., 2002). In this respect, it is important to note that the inclusion of separate practice sessions in the present study excludes any novelty effects. Furthermore, in the presented study, multiple reversal switches were assessed at each session as the reversal-learning task was designed to allow blocked fluorescence magnetic resonance imaging analyses in future research. It is always dangerous to draw conclusions based on 
negative results, especially when other factors may be involved, but the lack of ATD effects on reversal learning when novelty effects are excluded appears to be in line with the notion that the effects of ATD on probabilistic reversal learning may depend on task familiarity.

In conclusion, we have demonstrated that administration of a low-TRP collagen-based protein depletes peripheral TRP, decreases the peripheral TRP/ $/$ LNAA ratio and presumably diminishes TRP availability in the brain. The biochemical effects are very similar to those observed after a traditional ATD that is based on AA drinks, but with a more neutral placebo with regard to both TRP and TRP/ $\Sigma$ LNAA levels. Our data suggest that the CP method is a suitable alternative for the AA mixtures in TRP depletion research. Further studies will need to focus on the side-effect profile, optimal lag time for maximum biochemical and behavioral effects, and the validation of the method with regard to mood and cognitive changes in healthy, vulnerable and clinical populations.

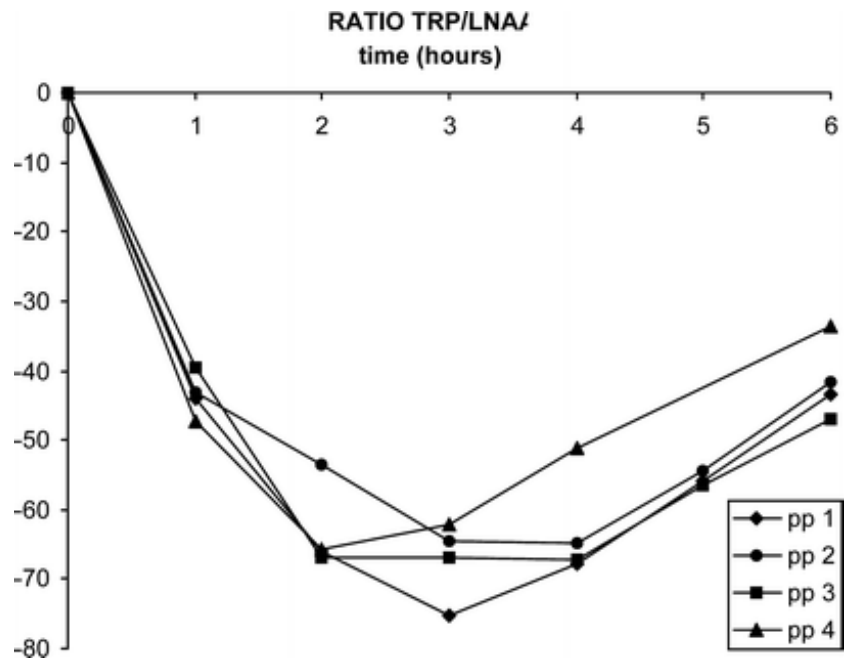

Figure 2. The ratio between tryptophan and the other large amino acids (TRP/ $\Sigma$ LNAA in percentage change) for four young healthy volunteers (p1-4) tested in a pilot experiment. Blood levels of TRP and the other large amino acids were measured at baseline and at hourly intervals until $6 \mathrm{~h}$ after consumption of the $\mathrm{TRP}^{-}$collagen protein mixture $(100 \mathrm{~g})$ 
Chapter 1 


\title{
Chapter 2
}

\section{Serotonergic Modulation of Prefrontal Cortex during Negative Feedback in Probabilistic Reversal Learning}

\author{
EAT Evers, R Cools, L Clark, FM van der Veen, J Jolles, BJ Sahakian, TW \\ Robbins
}

\begin{abstract}
This study used functional magnetic resonance imaging to examine the effects of acute tryptophan (TRP) depletion (ATD), a well-recognized method for inducing transient cerebral serotonin depletion, on brain activity during probabilistic reversal learning. Twelve healthy male volunteers received a TRP-depleting drink or a balanced amino-acid drink (placebo) in a double-blind crossover design. At $5 \mathrm{~h}$ after drink ingestion, subjects were scanned while performing a probabilistic reversal learning task and while viewing a flashing checkerboard. The probabilistic reversal learning task enabled the separate examination of the effects of ATD on behavioral reversal following negative feedback and negative feedback per se that was not followed by behavioral adaptation. Consistent with previous findings, behavioral reversal was accompanied by significant signal change in the right ventrolateral prefrontal cortex (PFC) and the dorsomedial prefrontal cortex. ATD enhanced reversal-related signal change in the dorsomedial PFC, but did not modulate the ventrolateral PFC response. The ATD-induced signal change in the dorsomedial PFC during behavioral reversal learning extended to trials where subjects received negative feedback but did not change their behavior. These data suggest that ATD affects reversal learning and the processing of aversive signals by modulation of the dorsomedial PFC.
\end{abstract}

\section{Acknowledgements}

We thank Wim Riedel and Ed Bullmore for helpful discussion, Matthew Brett for analysis support, Ruth Bisbrown-Chippendale and Clair Sleator from the Wolfson Brain Imaging Centre (Cambridge, UK) for radiographic assistance, and nursing staff of the Wellcome Trust Clinical Research Facility at Addenbrooke's hospital. This study was funded by a Wellcome Trust programme grant to TW Robbins, BJ Everitt, AC Roberts, and BJ Sahakian (LC), and completed within the MRC Centre for Behavioural and Clinical Neuroscience. EATE is funded by the ZonMW grant 912-02-050 from the dutch Organization for Scientific Research (NWO). RC holds a Royal Society Dorothy Hodgkin Fellowship and a Junior Research Fellowship from St John's College, Cambridge, UK.

Published in Neuropsychopharmacology 2005 Jun 30(6): 1138-47. 


\section{Introduction}

Serotonin (5-HT) has been extensively implicated in depressed mood and the processing of motivational signals (Graeff et al., 1986; Soubrie 1986; Deakin 1991; Wilkinson et al., 1995). Animal research has demonstrated that 5-HTenhancing drugs attenuate the aversive effects of brain stimulation (Patkina and Lapin, 1976; Graeff et al., 1986; Smith and Kennedy, 2003), and, conversely, potentiate self-stimulation in so-called 'reward' centers and enhance the motivational properties of stimuli predictive of rewards (Redgrave and Horrell, 1976; Aronson et al., 1995; Sasaki-Adams and Kelley, 2001; Orosco et al., 2004). Reduced motivation (anhedonia, apathy) is a cardinal feature of depression, where neuropsychological studies have further emphasized the relevance of incentive motivation and the processing of reinforcement; depression has been associated with a 'catastrophic response to perceived failure' (Beats et al., 1996) or an oversensitivity to negative feedback (Elliott et al., 1997; Steffens et al., 2001; Murphy et al., 2003). Selective 5-HT reuptake inhibitors exert antidepressant effects and acute reduction of central 5-HT function through dietary depletion of tryptophan (TRP), a precursor of 5-HT, can induce temporary depressive relapse in remitted patients (Young et al., 1985; Smith et al., 1997).

5-HT neurotransmission has been implicated not only in the processing of reward and punishment signals, but also in the inhibitory control of behavior (Soubrie, 1986; Evenden, 1999), where impulsive pathology is typically associated with reductions in central 5-HT (Coccaro et al., 1989; Cherek and Lane, 2000). Findings from studies with clinical populations are corroborated by animal studies linking impulsive choice in delay-discounting paradigms and premature responding in choice reaction-time tasks with 5-HT dysregulation (Harrison et al., 1997; Puumala and Sirvio, 1998; Koskinen et al., 2000; Mobini et al., 2000; Dalley et al., 2002; Liu et al., 2004).

These studies with animals have implicated particularly the medial and orbital prefrontal cortex (PFC) in impulsive performance (Dalley et al., 2002; Chudasama and Robbins, 2003; Liu et al., 2004), and this concurs indirectly with findings that manipulation of the 5-HT system in humans affects tasks that implicate the ventral and medial aspects of PFC (Robbins, 2000). Thus, neuropsychological studies have shown that acute TRP depletion (ATD) impairs performance on tasks of reversal learning, response inhibition, and affective decision-making (Park et al., 1994; Murphy et al., 2002; Walderhaug et al., 2002; Rogers et al., 2003), which have all been associated with ventral and/or medial PFC circuitry (Iversen and Mishkin, 1970; Jones and Mishkin, 1972; Dias et al., 1996; Rogers et al., 1999; O'Doherty et al., 2001; Cools et al., 2002; Fellows and Farah, 2003; Kringelbach and Rolls, 2003; Hornak et al., 2004). 
While the ascending 5-HT projection has a widespread cortical distribution, receptor subtypes including the 5- $\mathrm{HT}_{2 \mathrm{~A}}$ receptor show regional specificity to the frontal cortex and are overly expressed in medial and orbital regions in animal models of depression and anxiety (Poeggel et al., 2003; Preece et al., 2004). Structural and functional imaging studies in depressed patients also indicate reasonably selective abnormalities in the ventral and medial aspects of PFC (Drevets et al., 1997; Mayberg et al., 1999; Elliott et al., 2002; Ballmaier et al., 2004; Lacerda et al., 2004).

In the present study, we examined the effects of ATD on the blood oxygenation level-dependent (BOLD) response during probabilistic reversal learning, which requires the adaptation of behavior following changes in reward (and punishment) values as well as the maintenance of behavior in the face of misleading negative (probabilistic) feedback. ATD is a well-recognized research method for reducing central 5-HT in humans and studying the effects of low 5-HT on cognition. ATD produces a rapid decrease in the synthesis and release of brain 5-HT (Nishizawa et al., 1997; Carpenter et al., 1998; Williams et al., 1999). TRP is depleted by ingesting an amino-acid mixture that does not contain TRP but does include other large neutral amino acids (LNAA) (Young et al., 1985). ATD is achieved by increasing protein synthesis in the liver with subsequent decreases in plasma TRP stores. In addition, the amino-acid load results in competition for the active transport system that the amino acids share for entry across the blood-brain barrier, resulting in reduced availability of TRP in the brain. The probabilistic reversal learning task enables the relatively separate examination of behavioral adaptation following negative feedback (aversive signals) and the processing of negative feedback without subsequent behavioral adaptation. We used the same probabilistic reversal learning task that was previously employed by Cools et al. (2002). This study revealed significant BOLD changes during probabilistic reversal learning in the ventrolateral prefrontal cortex (VLPFC) and the dorsomedial PFC. Based on the strong a priori association between depression, 5HT, reversal learning, and orbital PFC, we decided not to restrict our regions of interest (ROIs) to the task-related brain areas but to extend these to the other orbital frontal regions not activated by the task. We predicted that ATD would modulate signal change in the ventral (including orbital) and medial PFC during the reception of negative feedback and the subsequent adaptation of behavior to the new contingencies. 


\section{Materials and methods}

\section{Participants}

Twelve healthy right-handed male volunteers (18-28 years old; mean age of $23.8 \pm 2.8$ ) participated in this experiment. The study was approved by the Local Research Ethical Committee in Cambridge and carried out in accordance with the Declaration of Helsinki. Participants were recruited via local advertisements, and screened for psychiatric and neurological disorders and MRI contraindications by means of prescreening questionnaires and interview by EATE. All volunteers gave written informed consent, and were paid for participation. The exclusion criteria were any history of cardiac, hepatic, renal, pulmonary, neurological or gastrointestinal disorder, medication use, and a history of major depression or bipolar affective disorder.

One participant vomited after ingesting the amino-acid mixture and was replaced by a substitute. One participant was excluded from the analysis due to poor performance on the reversal learning task (final $n=11$ ). His mean reaction time (RT) and the total number of trials on the reversal learning task were between 2.5 and 3.0 standard deviations higher than the group mean after the balanced drink.

\section{Experimental design}

Participants attended two test sessions at least 1 week apart, and were administered either a TRP-depleted (TRP-) drink or a balanced (BAL) amino-acid drink in a double-blind crossover design (four participants received TRP- and seven received the BAL drink on the first session). Prior to a test session, volunteers fasted overnight and low-protein food was provided during the test days. Following a resting period of $5 \mathrm{~h}(4.5 \mathrm{~h}, \mathrm{SD}=35 \mathrm{~min}$, in the TRP- condition and $5.0 \mathrm{~h}, \mathrm{SD}=40 \mathrm{~min}$, in the balanced condition), to ensure stable and low TRP levels (Riedel et al., 1999), participants entered the functional magnetic resonance imaging (fMRI) scanner at the Wolfson Brain Imaging Centre (WBIC). They were scanned while performing three blocks of the probabilistic reversal learning task each for about 9 min (Cools et al., 2002) and the checkerboard task. Behavioral performance on the reversal learning task was assessed using button presses on a response box. Structural scans were obtained at the end of a test session or on a separate session.

\section{Probabilistic reversal learning task}

The probabilistic reversal learning task was described in detail by Cools et al. (2002). The task is a two-choice visual discrimination task where the same two abstract patterns were presented on each trial. Using trial-and-error feedback after each response (a green happy face or a red sad face), subjects learned to select the 
stimulus that was usually correct. This rule intermittently reversed so that the other stimulus was usually correct. Consequently, responding had to be adjusted in order to gain reward and avoid punishment. On a minority of trials (10-20\%) falsenegative feedback was provided to a correct response, the so-called 'probabilistic errors' (0-4 per reversal). Reversal of the stimulus-reward contingency occurred after $10-15$ correct responses (including probabilistic errors). Participants performed three successive 9-min blocks of the task, each taking 140-160 trials (block length was determined by the number of errors made). Stimuli were presented for a $2000 \mathrm{~ms}$ response window (RTs $>2000 \mathrm{~ms}$ were followed by a 'too late' message). Feedback was presented immediately after the response for $500 \mathrm{~ms}$. After feedback, the stimuli were replaced by a fixation cross for a variable duration so that the overall interstimulus interval was $3215 \mathrm{~ms}$, enabling precise desynchronization from the repetition time (TR) of $1600 \mathrm{~ms}$.

Four types of events were modeled: (i) a correct response followed by positive feedback, (ii) a correct response followed by negative feedback (probabilistic error), (iii) an incorrect response where the subject reversed on the subsequent trial (reversal switch error), and (iv) an incorrect response where the subject did not reverse (ie perseverated) on the subsequent trial (preceding error). Spontaneous discrimination errors (those which could not be categorized as reversal or probabilistic errors) were not included in the model.

\section{Checkerboard task}

The checkerboard task was a passive visual task where the subject viewed two configurations of black and white squares in an $8 \times 8$ matrix that switched at a frequency of $8 \mathrm{~Hz}$. Using a blocked $\mathrm{ABAB}$ design, $20 \mathrm{~s}$ checkerboard blocks alternated with $20 \mathrm{~s}$ crosshair fixation for six cycles, taking a total of $4 \mathrm{~min}$.

\section{Amino-acid mixture}

The TRP-deficient amino-acid drink (TRP-) contained a total of $75 \mathrm{~g}$ of amino acids using the proportions described by Young et al. (1985): $4.1 \mathrm{~g}$ L-alanine, $2.4 \mathrm{~g}$ glycine, $2.4 \mathrm{~g}$ L-histidine, $6.0 \mathrm{~g}$ L-isoleucine, $10.1 \mathrm{~g}$ L-leucine, $6.7 \mathrm{~g} \mathrm{~L}$ lysine, $4.3 \mathrm{~g}$ L-phenylalanine, $9.2 \mathrm{~g}$ L-proline, $5.2 \mathrm{~g}$ L-serine, $4.3 \mathrm{~g}$ L-threonine, $5.2 \mathrm{~g}$ L-tyrosine, $6.7 \mathrm{~g}$ L-valine, $3.7 \mathrm{~g}$ L-arginine, $2.0 \mathrm{~g}$ L-cysteine, and $3.0 \mathrm{~g} \mathrm{~L}$ methionine (SHS International Ltd, Liverpool, UK). The balanced mixture contained the same amino acids, plus $3.0 \mathrm{~g}$ TRP. The drinks were prepared with $200 \mathrm{ml}$ tap water and fruit flavoring to compensate for the unpleasant taste.

\section{Biochemical measures}

Blood samples $(10 \mathrm{ml})$ were taken prior to ingestion of the amino-acid mixture and after the fMRI scan (about $6.5 \mathrm{~h}$ later), to determine the plasma TRP level and the TRP/ $\Sigma$ LNAA ratio. This ratio is important because the uptake of TRP in the 
brain is strongly associated with the amounts of other LNAA competing at the blood-brain barrier. Venous samples were taken in lithium heparin tubes, centrifuged, and stored at $-20^{\circ} \mathrm{C}$. Plasma TRP was determined by an isocratic high-performance liquid chromatography (HPLC) method of analysis. Plasma proteins were removed by precipitation with $3 \%$ trichloroacetic acid (TCA) and centrifugation at $3000 \mathrm{revs}, 4^{\circ}$ for $10 \mathrm{~min}$, and then pipetted into heparin aliquots. An aliquot was then diluted in mobile phase before injection into the HPLC analysis column. Fluorescence end-point detection was used to identify TRP.

Paired-sample t-tests were used to compare the two baseline measurements of plasma TRP levels and TRP/ $\Sigma$ LNAA ratios, and to compare measurements of plasma TRP levels and the TRP/SLNAA ratio in the balanced and TRP- condition. A repeated-measures ANOVA was performed to look at the effect of ATD on plasma TRP levels and the TRP/ $\Sigma$ LNAA ratios.

\section{Psychological ratings}

Visual Analogue Scales (VAS) containing the items drowsy, sad, happy, anxious and nauseous were administered five times during the test day (at roughly 90 min intervals). The Positive and Negative Affect Scale (PANAS; Watson et al., 1988) was completed prior to ingestion and after the scan. Repeated-measures ANOVA with drink treatment (TRP- and balanced) and time (two time points for the VAS, and two for the PANAS). Greenhouse-Geisser corrections were applied when the sphericity assumption was violated.

\section{Behavioral data analysis}

Dependent measures were the number of reversal contingencies during the task as a whole, the number of errors due to switching after a probabilistic error, mean $\mathrm{RT}$, and a maintenance score that was calculated by dividing the number of errors made following five correct responses but prior to the next contingency reversal by the number of trials remaining prior to the next contingency reversal (adapted from Swainson et al., 2000) for each reversal block. Data were analyzed using repeated-measures ANOVA with block (1-3) and treatment (balanced and TRP-) as within-subjects factors and order of drug treatment (balanced first or TRP- first) as between-subjects factor. Greenhouse-Geisser corrections were applied when the sphericity assumption was violated. Simple effects of block and treatment were analyzed using post hoc tests with Bonferroni correction for multiple comparisons. Medians were used for analysis because RTs were not normally distributed. Measures that were not normally distributed were analyzed with the nonparametric Wilcoxon signed ranks test. 


\section{Image acquisition}

Participants were scanned in a $3 \mathrm{~T}$ Bruker Medspec scanner (S300; Bruker, Ettlingen, Germany), at the WBIC. T2*-weighted gradient echo planner images (EPI) (TE $27 \mathrm{~ms}$ ) were acquired with blood oxygenation level-dependent (BOLD) contrasts. A whole-brain acquisition consisted of 21 slices (TR $1.6 \mathrm{~s}$; voxel size before normalization $1.56 \times 1.56 \times 5 \mathrm{~mm}^{3}$ and after normalization $3 \times 3 \times 3 \mathrm{~mm}^{3}$; inter-slice gap $1 \mathrm{~mm}$; matrix size 128 x 128; bandwidth $100 \mathrm{kHz}$; oblique orientation) and the total number of volumes acquired varied from run to run (from 142 to 166) depending on the participant's performance. In addition, highresolution T1-weighted images for spatial normalization were acquired of each participant (voxel size $1 \times 1 \times 1 \mathrm{~mm}^{3}$ ). We were unable to acquire reliable data from a section of ventromedial PFC because of susceptibility artifacts.

\section{Image analysis}

Data analysis was performed using SPM99 and SPM2 (Statistical Parametric Mapping; Wellcome Department of Cognitive Neurology, London, UK). Preprocessing procedures consisted of (linear) slice acquisition time correction, within-subject realignment (SPM2), geometric undistortion using fieldmaps (Cusack et al., 2003), spatial normalization using each individual subject's skullstripped SPGR (using the Brain Extraction Tool; Smith, 2002), and the Montreal Neurological Institute (MNI) skull-stripped structural template (SPM2) and spatial smoothing using a Gaussian kernel (10 mm full-width at half-maximum).

A canonical hemodynamic response was used as a covariate in a general linear model and a parameter estimate was generated for each voxel for each event type. For each event, the hemodynamic response function was modeled to the onset of the response, which co-occurred with the presentation of the feedback for the reversal learning task.

For each subject, the following contrasts were computed: (i) Main task effect 1: Reversal switch errors vs. baseline correct responses for the balanced condition only. (ii) Main task effect 2: Reversal nonswitch errors (which included probabilistic and preceding errors which were not followed by the subject switching responding) vs. baseline correct responses for the balanced condition only. (iii) Main task effect 3: Reversal switch errors vs. the other nonswitch errors for the balanced condition only. (iv) Treatment $\mathrm{x}$ task interaction 1, reflecting the effect of ATD on task effect 1. (v) Treatment $x$ task interaction 2, reflecting the effect of ATD on task effect 2. (vi) Treatment $x$ task interaction 3, reflecting the effect of ATD on task effect 3. Thus, treatment was modeled as a within-subject variable within each individual's general linear model. For the checkerboard task, an epoch (box-car) design contrasted checkerboard visual stimulation with crosshair fixation. Individual contrast images were taken to a second level analysis 
in which t-values were calculated for each voxel, treating inter-subject variability as a random effect.

The MarsBar tool (Brett et al., 2002) was used to average signal within independently defined ROIs at the group level. ROIs for the reversal task analysis were defined from the activation peaks found by Cools et al. (2002); $10 \mathrm{~mm}$ spheres (corresponding to the smoothing filter) were built around the dorsomedial PFC (x, y, z=8, 32, 52), right VLPFC (x, y, z=38, 24, -2) and left VLPFC (x, y, $\mathrm{z}=-32,24,-4)$. The random effects model was then reapplied to the average signal within these ROIs to test the statistical significance of the contrasts of interest (a one-sample t-test). Average signal change was extracted from each ROI and these are the values reported in Figure 2. In addition, we also performed whole-brain analyses. Both ROI and whole-brain analyses were thresholded at $\mathrm{P}<0.05$ (corrected for multiple comparisons). Given the a priori prediction concerning the modulation of the orbitofrontal cortex by ATD during reversal learning, we also examined the inferior, medial, and superior orbitofrontal cortex using ROIs from the Automated Anatomical Labelling (AAL) map based on the MNI average brain (Tzourio-Mazoyer et al., 2002), also thresholded at $\mathrm{P}<0.05$. Finally, the main effect of ATD was assessed by contrasting all task-related regressors from the TRP- condition with all task-related regressors from the balanced condition.

\section{Results}

\section{Functional imaging data}

All significant task-related effects from the balanced condition are shown in Table 1. ATD significantly increased the BOLD response in the dorsomedial PFC during reversal switch errors relative to correct baseline responses. This effect reached significance in both the ROI (contrast iv; $\mathrm{T}_{10}=2.04 ; \mathrm{P}=0.03$; Figure 1) and whole-brain analyses (Talairach coordinates $\mathrm{x}, \mathrm{y}, \mathrm{z}=9,39,48 ; \mathrm{T}_{10}=11.95$; $\left.P_{\text {corrected }}=0.006\right)$. However, the effect of ATD did not reach significance when the reversal switch errors were compared with the other nonswitch errors (contrast vi; $\left.\mathrm{T}_{10}=1.33 ; \mathrm{P}=0.1\right)$. Furthermore, the increase in signal in the dorsomedial PFC tended towards significance when the reversal nonswitch errors were compared with baseline correct responses (contrast $\mathrm{v} ; \mathrm{T}_{10}=1.44 ; \mathrm{P}=0.09$ ) (Figure 2). Thus, ATD increased signal changes during all negative feedback, irrespective of whether the errors were followed by behavioral reversal. ATD did not affect the BOLD response in the left and right VLPFC $\left(\mathrm{T}_{10}=-0.13, \mathrm{P}=0.55\right.$ and $\mathrm{T}_{10}=-0.21$, $\mathrm{P}=0.6$, respectively). Furthermore, ATD did not significantly affect global activation changes during the task, as revealed by both whole-brain and ROI analyses (ROI analyses: left VLPFC: $\mathrm{P}=0.33$; right VLPFC: $\mathrm{P}=0.32$; medial PFC: $\mathrm{P}=0.23$ ). 


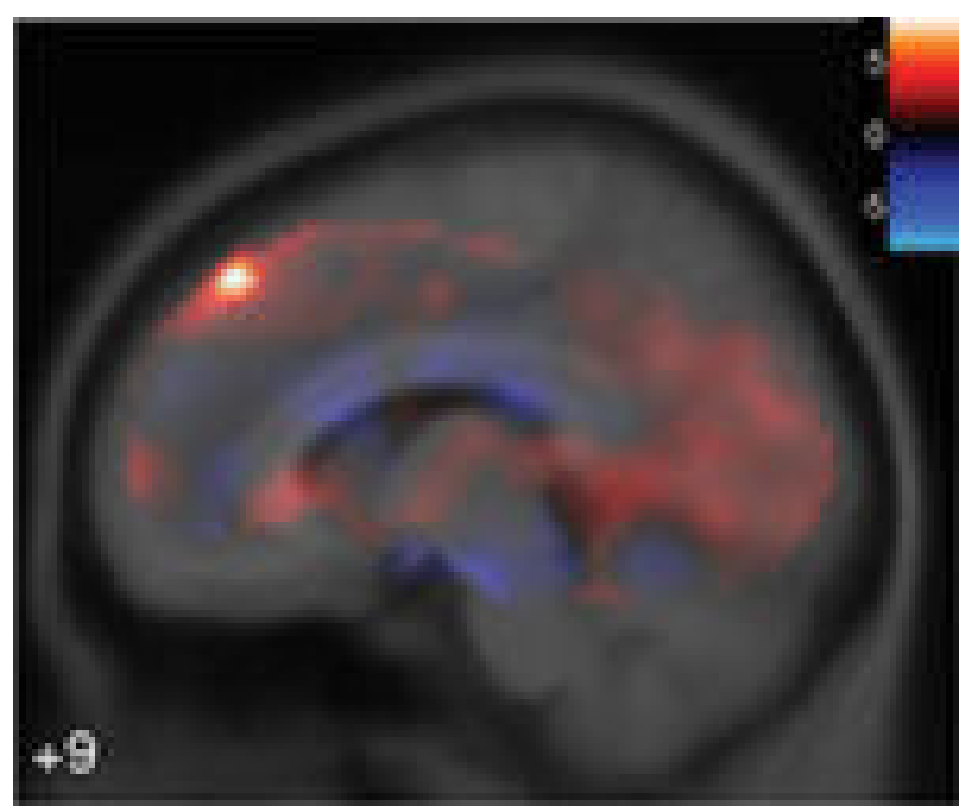

Figure 1. The continuous t-value image (statistical parametric map) is shown as a sagittal section (MNI coördinate $\mathrm{y}=9$ ) superimposed upon the average MNI T1 template (152 brains). The t-map represents functional activation changes following ATD relative to the balanced condition during reversal switch errors relative to baseline correct responses (a treatment $\mathrm{x}$ task interaction effect). Whole-brain (and ROI) analyses revealed a significant signal increase following TRP depletion in the dorsomedial PFC, centered on MNI coördinates $(\mathrm{x}, \mathrm{y}, \mathrm{z})=(9,39,48)\left(\mathrm{T}_{10}=11.95\right.$, $\mathrm{P}_{\text {corrected }}=0.006$ ).

Table 1. Significant task effects revealed by regions of interest analyses.

\begin{tabular}{lcc}
\hline Increased activation & T-values & $\mathrm{P}$ \\
\hline Reversal switch errors minus correct responses (contrast $i)$ & 4.22 & $<0.001$ \\
Dorsomedial PFC & 7.02 & $<0.001$ \\
Right ventrolateral PFC & 3.43 & $<0.01$ \\
Left ventrolateral PFC & & \\
Other non-switch errors minus correct responses (contrast ii) & 4.83 & $<0.001$ \\
Right ventrolateral PFC & 3.16 & $<0.01$ \\
Left ventrolateral PFC & 5.15 & $<0.001$ \\
Reversal switch errors minus other non-switch errors (contrast iii) & $<.55$ & $<0.001$ \\
Dorsomedial PFC & 4.55 \\
Right ventrolateral PFC &
\end{tabular}



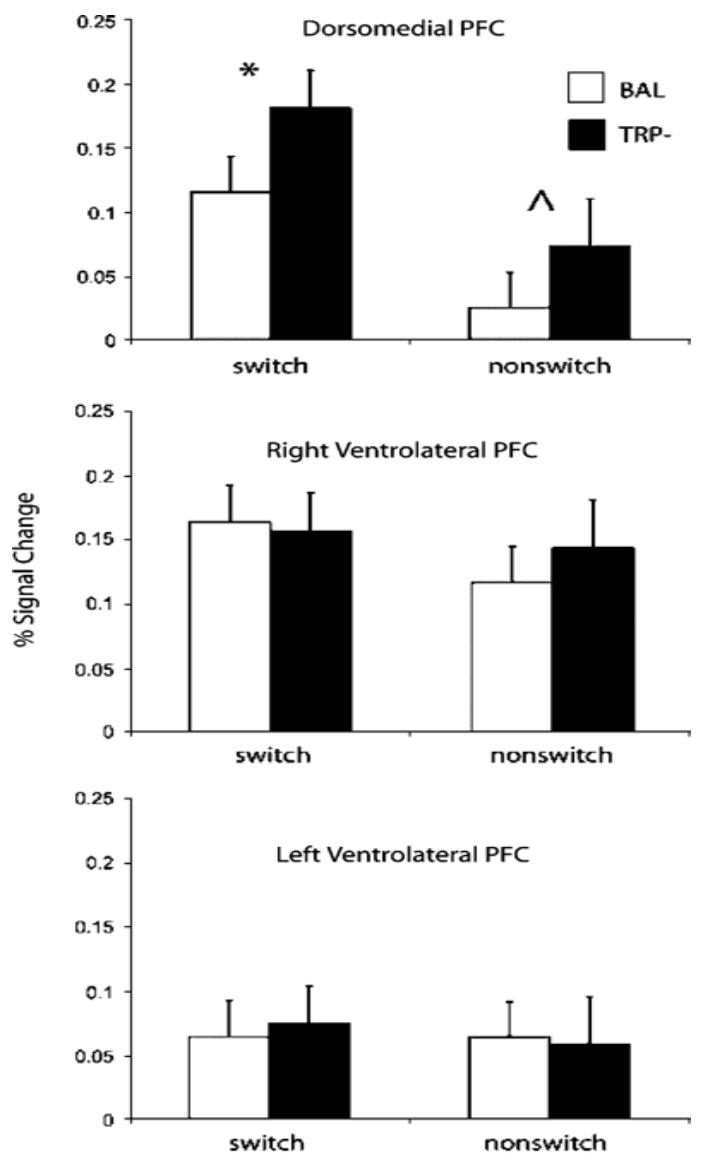

Figure 2. Percent signal change is shown for three ROI: the dorsomedial PFC, the right and the left ventrolateral PFC for reversal switch errors (relative to baseline correct responses), and nonswitch errors (including probabilistic and preceding errors relative to baseline correct responses) in the BAL and TRP- conditions. Errors bars represent SEMs. ROI (spheres with $10 \mathrm{~mm}$ radius) were defined from peak coordinates from Cools et al. (2002) (see Materials and methods section for coordinates). ${ }^{*} P<0.05, \wedge P<0.1$.

While an ATD-induced increase in the orbitofrontal cortex during the reversal switch errors compared with correct responses did not reach significance in the ROI or whole-brain analysis according to our criterion (AAL's left middle orbital 
gyrus: $\mathrm{T}_{10}=1.12 ; \mathrm{P}=0.14$ ), for completion we report that whole-brain analysis revealed a nonsignificant effect at $\mathrm{x}, \mathrm{y}, \mathrm{z}=-48,42,-3\left(\mathrm{~T}_{10}=5.72 ; \mathrm{Z}=3.7\right)$.

Supplementary analysis revealed that our findings are not confounded by the fact that four participants started with the TRP- condition and seven participants started with the balanced condition. This analysis of the individual parameter estimates, extracted from the dorsomedial PFC ROI (reversal switch errors minus baseline correct responses), revealed that the effect of ATD was not qualified by testing order (ATD $x$ testing order interaction: $\mathrm{F}_{1,9}=0.5, \mathrm{P}=0.5$ ). No differences in signal change were observed between subjects who ingested the TRP- drink on the first occasion (mean signal change $=0.16$ ) and subjects who ingested the TRPdrink on the second occasion (mean signal change $=0.19$ ), and no differences were observed between subjects who ingested the balanced mixture on the first occasion (mean signal change $=0.11$ ) and subjects who ingested the balanced mixture on the second occasion (mean signal change $=0.12$ ). Whole-brain analyses did not reveal any other significant effects.

\section{Behavioral effects of ATD on reversal learning}

There was a nonsignificant tendency for ATD to slow overall RT $\left(\mathrm{F}_{1,9}=4.72\right.$, $\mathrm{P}=0.06$ ); mean $\mathrm{RT}$, TRP- $=554 \mathrm{~ms}, \mathrm{BAL}=522 \mathrm{~ms}$ ). No other differences were found between the TRP- and balanced condition. Mean values are presented in Table 2.

Table 2. Behavioral effects of ATD

\begin{tabular}{lcc}
\hline & Balanced & TRP- \\
\hline Total number of reversals & $26.8(0.1)$ & $26.1(0.5)$ \\
$\begin{array}{l}\text { Total number of switches after } \\
\text { probabilistic errors }\end{array}$ & $10.3(2.8)$ & $10.3(2.9)$ \\
$\begin{array}{l}\text { Mean maintenance score } \\
\text { Mean overall RT }\end{array}$ & $0.06(0.0)$ & $0.06(0.1)$ \\
\hline
\end{tabular}

Values represent means (standard errors of the mean); ${ }^{\wedge}$ The effect of ATD on mean RT tended towards significance at $P=0.06$

\section{Checkerboard task}

Visual stimulation was associated with a large and highly significant activation cluster $(\mathrm{T}=13.63, \mathrm{P}=0.002)$ in the occipital cortex. No effects of ATD on visual stimulation in this region in occipital cortex were found, even at $\mathrm{P}=0.01$ uncorrected for whole-brain volume. 


\section{Biochemical measures}

The analysis of amino-acid levels revealed that the depletion was successful. No differences between conditions were found in terms of plasma TRP level (mean/SEM: BAL 12.2/2.3; TRP 12.3/3) and ratio TRP/ $2 L N A A$ 's (BAL 0.19/0.02; TRP- 0.16/0.02) at baseline. After $6.5 \mathrm{~h}$, plasma TRP and the ratio TRP/ $/$ LNAA were significantly lower in the TRP- condition compared with the balanced condition (TRP levels: BAL 18.2/6.3; TRP- 4.4/1.8; $\mathrm{T}_{10}=8.3, \mathrm{P}<1$ and ratios: BAL 0.16/0.03; TRP- 0.04/0.01; $\mathrm{T}_{10}=4.0, \mathrm{P}<0.01$, respectively). Following the TRP- drink, plasma TRP was reduced by $64 \%$ and the ratio TRP/ $/$ LNAA's by $74 \%$ relative to baseline t0. Following the balanced drink, the plasma TRP was increased by $50 \%$ and the ratio TRP/ $/$ LNAA was reduced by $16 \%$.

\section{Psychological ratings}

No interaction effects of ATD by time on PANAS-positive and -negative effect scores were observed (positive effect: $\mathrm{F}_{1,10}=1.1, \mathrm{P}=0.3$; negative effect: $\mathrm{F}_{1,10}=1.0$, $\mathrm{P}=0.3$ ), while there were significant main effects of time across drink treatment on both scores (positive effect: $\mathrm{F}_{1,10}=6.7, \mathrm{P}=0.03$; negative effect: $\mathrm{F}_{1,10}=6.1, \mathrm{P}=0.03$ ) (Table 3). Similarly, while some main effects of time reached significance for VAS scores (Drowsiness increased over time: $\mathrm{F}_{1,10}=14.7, \mathrm{P}=0.003$; Happiness and anxiety decreased over time: $\mathrm{F}_{1,10}=011.3, \mathrm{P}=0.007$ and $\mathrm{F}_{1,10}=11.1, \mathrm{P}=0.008$, respectively), no significant interaction effects between time and drink treatment on VAS scores were observed (Drowsiness: $\mathrm{F}_{1,10}=0.04, \mathrm{P}=0.8$; Sadness: $\mathrm{F}_{1,10}=0.7$, $\mathrm{P}=0.4$; Happiness: $\mathrm{F}_{1,10}=1.0, \mathrm{P}=0.3$; Anxiety: $\mathrm{F}_{1,10}=1.6, \mathrm{P}=0.2$; Nausea: $\mathrm{F}_{1,10}=0.03$, $\mathrm{P}=0.9$ ).

Table 3. Psychological Ratings of mood at baseline t0 and post-scanning t6.5

\begin{tabular}{cllll}
\hline & BAL t0 & BAL t6.5 & TRP- t0 & TRP- t6.5 \\
\hline$V A S$ & & & & \\
Drowsy & $2.0(2.1)$ & $3.1(2.3)$ & $2.1(1.8)$ & $3.4(3.1)$ \\
Sad & $1.0(0.7)$ & $1.8(1.6)$ & $1.8(1.6)$ & $2.0(1.6)$ \\
Happy & $5.2(2.0)$ & $4.0(2.3)$ & $5.5(1.5)$ & $5.0(1.4)$ \\
Anxious & $2.6(1.8)$ & $1.2(1.5)$ & $1.9(1.4)$ & $1.4(1.0)$ \\
Nausea & $1.6(2.0)$ & $1.4(1.1)$ & $1.9(2.0)$ & $1.7(1.3)$ \\
PANAS & & & & \\
Positive & $26.9(1.9)$ & $23.7(1.6)$ & $28.6(0.9)$ & $23.6(1.8)$ \\
Negative & $13.4(0.8)$ & $11.8(0.5)$ & $13.4(0.7)$ & $13.3(0.8)$ \\
\hline
\end{tabular}

$\mathrm{BAL}=$ balanced condition $; \mathrm{TRP}=$ tryptophan depleted condition 


\section{Discussion}

The present findings reveal that acute depletion of central 5-HT in young healthy male volunteers significantly increased the task-related BOLD response in the dorsomedial PFC during probabilistic reversal learning. By contrast, ATD did not modulate task-related signal change in the ventrolateral PFC. These effects contrast with those observed following manipulation of the central dopamine system in young healthy volunteers, which reduced the BOLD signal in the left ventrolateral PFC during reversal learning but did not affect signal in the dorsomedial PFC (Clark et al., 2004), and suggest that changes in the 5-HT system affect probabilistic reversal learning via action in the dorsomedial PFC.

The observed reduction in plasma TRP was comparable to that seen in previous studies (Young et al., 1985; Murphy et al., 2002) and data from both animal and human studies indicate that acute precursor depletion induces significant reductions in the synthesis and release of brain 5-HT (Biggio et al., 1974; Nishizawa et al., 1997; Carpenter et al., 1998). The BOLD changes following ATD are unlikely to reflect indirect effects of the dietary manipulation on mood or arousal, because no effects of ATD on any of the subjective ratings were observed. As predicted, ATD did not affect depressive symptomatology in this sample of young volunteers without a history of psychiatric disorder (Benkelfat et al., 1994). Although there was a tendency towards an overall slowing of response latencies following depletion of central 5-HT, the BOLD changes were not accompanied by significant behavioral changes and are thus unlikely to reflect increased task difficulty. The observed tendency towards a behavioral effect concurs with previous observations that ATD induces significantly slower latencies in a neuropsychological version of the probabilistic reversal learning task (Murphy et al., 2002), which involved only a single reversal of contingencies. Murphy et al. (2002) showed that ATD slowed responding only in a first test session, in which the task was novel to the subjects. In light of these previous observations, we suggest that the present behavioral measure of repeated, well-practiced and serial reversal learning was not sufficiently sensitive to reveal significant behavioral effects of ATD. Furthermore, we propose that the physiological BOLD response provided a more sensitive measure of the effects of ATD, hypothetically accompanying behavioral changes that surface only in more sensitive, single reversal learning paradigms.

The increased response in the dorsomedial PFC after 5-HT depletion was significant when the reversal switch errors were compared with correct responses, but did not reach significance when the reversal switch errors were compared with the reversal nonswitch errors. Moreover, ATD increased (although only marginally significantly) the BOLD signal during the reversal nonswitch errors relative to correct responses. The ATD effect was therefore not restricted to errors 
that were followed by a behavioral switch, but extended to the other error types where negative feedback was presented without subsequent reversal. The present task thus implicates 5-HT in processes other than reversal switching, which may include the detection/monitoring of errors and/or conflict. This is consistent with cognitive models of medial frontal cortex function, which emphasize the region's involvement in error detection and conflict monitoring (Paus et al., 1993; Carter et al., 1998; Gehring and Fencsik, 2001; Kerns et al., 2004; MacDonald et al., 2000; Garavan et al., 2003).

Based on evidence for a role of 5-HT in the processing of aversive signals, as well as models of depression as an oversensitivity to negative feedback (Elliott et al., 1997; Steffens et al., 2001; Murphy et al., 2003; Graeff et al., 1986; Smith and Kennedy, 2003), we hypothesize that the increased BOLD signal in the dorsomedial PFC reflects enhanced processing of aversive signals (error feedback) leading to enhanced response conflict on both switch and nonswitch trials. Clearly, the task does not fully dissociate negative feedback, conflict and behavioral switching: negative feedback initiates conflict and subsequent reversal, and a probabilistic task places demands on inhibitory control to maintain responding after misleading feedback. This is illustrated by the findings of Murphy et al. (2003), showing that depressed people exhibit impaired maintenance of responding in the probabilistic reversal learning task, specifically following misleading negative feedback. Serotonergic modulation of medial frontal function is also consistent with the selective abnormalities seen in this region in structural imaging and post-mortem investigations in mood disorders (Drevets et al., 1997; Rubinsztein et al., 2001; Merali et al., 2004).

Altered 5-HT neurotransmission in the PFC has also been associated with failures of inhibitory control (Leyton et al., 2001; Clarke et al., 2004; Liu et al., 2004). A recent study has demonstrated increased perseverative responding in marmosets following prefrontal 5-HT depletion, using a simple (nonprobabilistic) reversal learning task (Clarke et al., 2004). There are significant methodological differences between the tasks used in that study and the current fMRI task. Primarily, the probabilistic nature of the fMRI task increases the incidence of error processing and is likely to enhance the salience of punishment during the task. This may render the task particularly sensitive to changes in punishment sensitivity. The nature of punishment is also different in the two tasks. The fMRI task employs a specific error signal (a red sad face), whereas reversal is indicated to the marmoset by the omission of reward for the previously relevant stimulus, co-occurring with unexpected reward for the newly relevant stimulus. The hypothesized increase in sensitivity to negative feedback induced by ATD in the current study may coexist with a deficit in the inhibitory control of behavior. In probabilistic reversal learning, this may diminish the ability to maintain responding to the relevant stimulus following misleading negative feedback, as 
shown in depressed patients by Murphy et al. (2003). However, in a nonprobabilistic reversal learning task that de-emphasizes the processing of punishment cues, behavioral changes may surface as impaired inhibitory control, in the form of perseverative responding. Of course, it also remains to be established at the present time whether a global depletion of 5-HT precursor in humans is functionally equivalent to a prefrontal lesion of 5-HT fibres in marmosets.

The present pattern contrasts with effects of the (dopamine) DA D2 receptor antagonist as well as the indirect catecholamine agonist methylphenidate, which resulted in significant reductions in the BOLD signal in the ventrolateral PFC that were specific to behavioral switching and cannot have been solely induced by the reception of negative feedback (Clark et al., 2004). This dissociation concurs with the observation that the indoleamine 5-HT modulates in particular a medial and orbital prefrontal neural system associated with associative learning and affective decision making, while catecholamines (ie DA and norepinephrine) affect predominantly a system connecting to the lateral PFC associated with set shifting, response inhibition, and working memory (Robbins, 2000; Aron et al., 2004).

The PFC is densely innervated with large ascending serotonergic projections from the raphé nuclei and there are several mechanisms via which 5-HT may modulate PFC neuronal responses. For example, evidence from in vitro studies show that activation of $5-\mathrm{HT}_{2 \mathrm{~A}}$ receptors induces a rapid increase in excitatory postsynaptic potentials/currents in virtually all layer $\mathrm{V}$ pyramidal cells of neocortex, but most prominently in medial PFC and other frontal regions where 5$\mathrm{HT}_{2 \mathrm{~A}}$ receptors are enriched (Marek and Aghajanian, 1998). Activation of postsynaptic $5-\mathrm{HT}_{1 \mathrm{~A}}$ receptors in the $\mathrm{PFC}$, on the other hand, have been found to inhibit pyramidal cell activity (Puig et al., 2004). It is unlikely that our findings reflect vasoconstrictive or vasodilative effects of ATD leading to indirect (nonneuronal) changes in the hemodynamic response, because the increase was regionally selective to dorsomedial PFC and did not extend to other task-related brain areas, which were modulated by dopaminergic drugs. Vascular effects would be predicted to affect task-related signal globally. Moreover, ATD did not affect significantly task-related signal during the presentation of a checkerboard task that elicited a robust response in visual striate cortex.

The present study only used male volunteers. Previous studies showed that females are more sensitive to mood effects after ATD than males (Ellenbogen et al., 1996) and thus male volunteers were selected for the present study to avoid the potentially confounding effects of mood on task performance and task-related BOLD signal change. However, the cognitive and biological effects of 5-HT depletion may be markedly greater in female subjects (Nishizawa et al., 1997; Harmer et al., 2003). For example, ATD reduced the rate of 5-HT synthesis by 40 times in females compared to only 10 times in males (Nishizawa et al., 1997). Our 
results might be representative for a male population only, and need to be extended to female subjects in subsequent research. In a further feature of the experimental design, we employed a $75 \mathrm{~g}$ TRP- mixture rather than the more typical $100 \mathrm{~g}$ mixture, in order to minimize the incidence of nausea and vomiting during the scanning procedure. The biochemical analyses confirmed a successful depletion with this reduced volume, whereby plasma TRP was reduced by $64 \%$ on average, and the ratio of TRP to other LNAA was reduced by $74 \%$. The ratio of TRP/ $/$ LNAA was virtually unaffected by the balanced mixture (only a $16 \%$ decrease relative to baseline), and this ratio is associated most closely with reduced 5-HT synthesis.

In conclusion, acute depletion of brain 5-HT levels in healthy male volunteers increased the BOLD signal in dorsomedial PFC during performance of a task that involves feedback processing and the adaptation of behavior. The effects of 5-HT were related to the processing of negative feedback more than behavioral adaptation per se, and this provides one of the first direct demonstrations of serotonergic modulation of medial frontal processes in human subjects. During reversal learning, the dorsomedial PFC response is part of an extended network of frontal activity, and these data highlight the potential of pharmacological fMRI as a tool for dissecting the neurochemical contributions to cognitive control mechanisms. 


\title{
The effect of acute tryptophan depletion on the BOLD response during performance monitoring and response inhibition in healthy male volunteers.
}

\author{
EAT Evers, FM van der Veen, JA van Deursen, JAJ Schmitt, NEP Deutz, J Jolles
}

\begin{abstract}
Rationale. Serotonin (5-HT) has been implicated in both clinical and experimental studies in flexible goal-directed behavior. However, the way in which 5-HT manipulations affect brain activation patterns underlying different sub-processes of cognitive flexibility remains largely unknown. Objectives. The aim of this study was to investigate the effect of a transient lowering of 5-HT on brain activation during performance monitoring and response inhibition. Methods. We used acute tryptophan depletion (ATD), a well-known method to reduce central 5-HT, to investigate the effect of a transient lowering of 5-HT on the Blood Oxygen Level Dependent (BOLD) response in an event-related fMRI study. Thirteen healthy male volunteers performed a modified Go/NoGo task in a counterbalanced placebo-controlled within-subject design. Results. ATD significantly lowered plasma tryptophan, but did not affect mood and cognitive performance. ATD decreased the BOLD response in the dorsomedial prefrontal cortex (BA 8) during performance monitoring. ATD did not affect the BOLD response during response inhibition. Conclusions. This study provides more evidence for the suggested role of 5-HT in performance monitoring. Since ATD studies have revealed inconsistent effects of ATD on performance and on brain activation, it was suggested that gender and personality trait are important variables to take into account for future research.
\end{abstract}

\section{Acknowledgements}

This work was supported by a TOP grant (No. 912-02-050) from ZonMW-NWO. This study complies with the Dutch laws for human research and was approved by the Medical Ethics Committee of the Maastricht University Hospital.

In press in Psychopharmacology (Berl) 


\section{Introduction}

Previous animal and human research showed that serotonin (5-HT) is implicated in cognitive flexibility tasks (for review Robbins 2005). Cognitive flexibility is the ability to quickly adapt performance to task related changes. It has been shown that acute tryptophan depletion (ATD), a method to temporarily reduce brain 5-HT levels, impaired performance during reversal learning (Park et al., 1994; Rogers et al., 1999a) and decision making (Rogers et al., 1999b; 2003) in healthy volunteers. In contrast, other studies did not find an effect of reduced 5HT on cognitive flexibility (Anderson et al., 2003; Talbot et al., 2006). Several cognitive processes are important for cognitive flexibility, for example performance monitoring and response inhibition. It remains unclear how these processes are affected by a transient lowering of 5-HT. The present study investigated the effect of low 5-HT on performance and brain activation during performance monitoring and response inhibition.

Previous studies showed that low 5-HT impaired performance monitoring and response inhibition. For example, patients suffering from unipolar depression, a disorder associated with disturbed 5-HT functioning (for review see Risch and Nemeroff, 1992), showed an impaired response to negative feedback. Two previous studies (Beats et al., 1996; Elliott et al., 1997) reported that depressed patients fail to improve performance after perceived failure, which suggests cognitive inflexibility. Murphy et al. (2003) showed impaired ability to maintain a response set in the face of misleading negative feedback in depressed patients, which indicates an increased tendency to change response strategy. Fallgatter et al. (2004) investigated the modulation of the brain electrical response to error processing as a function of allelic variation of 5-HT transporter function. They showed a significantly higher amplitude of the error-related negativity, indicating an enhanced responsiveness of the anterior cingulate cortex (ACC) during error processing, when participants carried a short allele of the 5-HT transporter. Animal studies showed that the administration of the neurotoxin 5,7dihydroxytryptamine, depleting brain 5-HT, resulted in poorer response inhibition on a Go/NoGo task (Harrison et al., 1999) and increased impulsivity in a fivechoice serial reaction time task (Harrison et al., 1997; Winstanley et al., 2004) in rats. Furthermore, depressed patients showed disturbed response inhibition on a Go/NoGo task (Kaiser et al., 2003). In contrast to the suggested importance of 5HT for response inhibition, no effects of ATD were found on response inhibition performance in healthy volunteers (Clark et al., 2005; Cools et al., 2005a; Crean et al., 2002; Le Marquand et al., 1998, 1999; Rubia et al., 2005). Only one study that we are aware of showed an increase in impulsive response style after ATD (Walderhaug et al., 2002). 
Three previous fMRI studies investigated the effect of ATD on brain activation while healthy volunteers performed a cognitive task. Horacek et al. (2005) showed that ATD increased activation in the bilateral medial, inferior and superior prefrontal cortex (PFC) and in the ACC during the interference condition of a Stroop task. Rubia et al. (2005) investigated the effect of ATD on brain activation during response inhibition using a standard Go/NoGo task. ATD increased the Blood Oxygen Level Dependent (BOLD) response in the superior and medial temporal cortices, but decreased the BOLD response in the right inferior PFC during response inhibition. Finally, Evers et al. (2005) showed that ATD increased the BOLD response in the dorsomedial PFC (dmPFC) during probabilistic reversal learning. In addition, two PET studies have examined the effect of ATD on the activation of a resting brain in depressed patients (Bremner et al., 1997; Smith et al., 1999; Morris et al., 1999). A diverse range of areas was affected by ATD including the orbitofrontal cortex, the ACC, the caudate, raphe and habenula nucleus, the thalamus and the dorsolateral PFC.

The aim of the current study was to investigate the effect of a transient lowering of 5-HT, by means of ATD, on brain activation patterns underlying performance monitoring and response inhibition. ATD is a well-established, noninvasive method that adequately reduces the release and synthesis of brain 5-HT in humans (Biggio et al., 1974; Fadda et al., 2000; Williams et al., 1999; Nishizawa et al., 1997). In the present study healthy male volunteers performed a modified Go/NoGo task in which performance feedback was given after each response, in an event-related fMRI design. In accordance with above mentioned studies that showed that ATD did not affect response inhibition in healthy volunteers, we hypothesized that performance would not be affected by ATD. Furthermore we hypothesized that ATD affects the BOLD response in dorsomedial frontal regions (based on Evers et al., 2005; Fallgatter et al., 2004) during performance monitoring and in the right inferior frontal cortex (based on Rubia et al., 2005) during response inhibition.

\section{Materials and methods}

\section{Participants}

Seventeen healthy right-handed male volunteers were included in this study, which was approved by the Medical Ethics Committee of the Maastricht University Hospital. Data of four volunteers could not be used for analysis due to technical problems. The remaining participants $(n=13)$ were on average 23 years old $(\mathrm{SD}=1.8)$, and were mostly pregraduate students. Participants were recruited by local advertisements and were not suffering from significant past or present 
physical or psychiatric illnesses, which was checked by a medical health questionnaire. They received no medication at the moment of inclusion and were screened for MRI contra-indications. This study has been carried out in accordance with the declaration of Helsinki and all volunteers gave written informed consent prior to their inclusion in the study. The participants were paid 75 euros for participation.

\section{Experimental design}

Participants were scanned at the Academic Hospital Maastricht on two separate test days, that is an experimental and a placebo test session counterbalanced according to a double-blind crossover design. Participants arrived in the afternoon, after they had fasted overnight and eaten a low protein breakfast at home. They were given a tryptophan-depleted (TRP-) or a balanced (BAL) AA mixture, followed by a five-hour break to ensure stable and low plasma TRP levels (Riedel et al., 1999). In this break only low protein food was eaten. In the evening (between 5 and $8.30 \mathrm{pm}$ ) the participants were scanned while performing two blocks of the Go/NoGo task, each lasting about 5.5 minutes. The participants also performed a memory task but the results of this part of the experiment will be presented elsewhere (van der Veen et al., 2006). The order of testing was as follows: the first $\mathrm{Go} / \mathrm{NoGo}$ block $(5.5 \mathrm{~min})$, the encoding block of the memory task (7.5 $\mathrm{min})$, the second Go/NoGo block (5.5 min), the_structural scan (10 $\mathrm{min})$ and the recognition block of the memory task $(11 \mathrm{~min})$. Participants were trained in a dummy scanner on a separate day before the first test sessions to make sure that they were comfortable in the scanner and perform the task well.

\section{The Go/NoGo task}

The present study used a modified version of the Go/NoGo task described by Garavan et al. (1999). The task was programmed in E-Prime V1.0 (Psychology Software Tools, 2002). A stream of letters (yellow on a black background), was presented, showing one letter every $500 \mathrm{~ms}$, with a $0 \mathrm{~ms}$ inter stimulus interval. The participants' task was to respond to an X preceded by a Y, or a Y preceded by an $\mathrm{X}$ (Go trials) by pressing a button on a response box with their right index finger. Participants had to refrain from responding when an $X$ was preceded by an $\mathrm{X}$, or a Y by a Y (NoGo or inhibition trials). Feedback, which started $500 \mathrm{~ms}$ after the presentation of a letter (fixed delay) and was presented for $500 \mathrm{~ms}$, was added to the original task design to motivate the participants. Correct Go and NoGo trials were followed by a green square (positive feedback), incorrect responses were followed by a red square (negative feedback). Negative feedback was given after a response to an inhibition trial, a response to a letter other than an X or a $\mathrm{Y}$, no response to a Go trial, or a response was given after a time limit of $500 \mathrm{~ms}$. This time limit was added to assure fast responding. The response had to be made when 
the stimulus was still on the screen. Feedback was given after the stimulus presentation. A NoGo trial was presented on average every 25 seconds and a Go trial on average every 4 seconds. In each test session 1000 stimuli were presented, of which 25 NoGo and 150 Go trials, divided into two blocks each lasting about 5 minutes. Four different versions were used in a randomized order. Participants were trained before the actual testing. In the first training task no NoGo trials (400 stimuli, of which 120 Go trials), and in the second training task seven NoGo trials (350 stimuli, of which 55 Go trials and 7 NoGo trials) were presented in addition to the Go trials. The experimental task contained three events of interest: correctly executed Go trials followed by positive feedback (Go's) correctly inhibited NoGo trials followed by positive feedback (NoGo's) and errors on Go or NoGo trials followed by negative feedback (Errors).

\section{Performance data}

The following measures were used: the number of correct Gos, the number of correct NoGos, the mean reaction time (RT) on Go trials after a correct response (a measure for general responding speed), after an incorrect Go and after an incorrect NoGo trial, (measures for the behavioral adaptation after a response error). The effect of ATD on number of correct Gos, the number of correct NoGos and the RT measures was analyzed (SPSS version 11.5 for windows) using repeated measures ANOVA with Treatment (BAL or TRP-) as a within subject variable and Order (TRP- or BAL mixture first) as a between subject variable. The differences between the RT measures within the BAL and TRP- condition were analyzed using paired-sample t-tests.

\section{Amino acid (AA) mixtures}

The TRP-deficient (TRP-) amino acid (AA) mixture contained $75 \mathrm{~g}$ of AAs with the amounts of each specific AA according to the following proportions: 4.1 g L-alanine, $2.4 \mathrm{~g}$ L-glycine, $2.4 \mathrm{~g}$ L-histidine, $6.0 \mathrm{~g}$ L-isoleucine, $10.1 \mathrm{~g} \mathrm{~L}$ leucine, $6.7 \mathrm{~g}$ L-lysine, $4.3 \mathrm{~g}$ L-phenylalanine, $9.2 \mathrm{~g}$ L-proline, $5.2 \mathrm{~g}$ L-serine, 4.9 g L-threonine, $5.2 \mathrm{~g}$ L-tyrosine, $6.7 \mathrm{~g}$ L-valine, $3.7 \mathrm{~g}$ L-arginine, $2.0 \mathrm{~g}$ L-cysteine, $2.3 \mathrm{~g}$ L-methionine. The nutritionally balanced (BAL) mixture contained the same amount of these AAs, plus $3.0 \mathrm{~g}$ TRP. The mixtures were prepared with $200 \mathrm{ml}$ tap water.

\section{Biochemical measures}

Blood samples $(10 \mathrm{ml})$ were taken immediately before ingestion of the AA mixture and just before the scanning session, about four and a half hours after administration, to determine the plasma TRP level and the TRP/ $\Sigma$ LNAA ratio. The TRP/LLNAA ratio was calculated as follows: (TRP) / (tyrosine + leucine + phenylalanine + isoleucine + valine $)$. The blood samples were immediately 
centrifuged at $4^{\circ} \mathrm{C}$ (10 minutes, $\left.4500 \mathrm{rpm}\right)$. Hundred $\mu \mathrm{l}$ aliquot of plasma was mixed with $8 \mathrm{mg}$ sulfa salicylic acid and frozen at $-80{ }^{\circ} \mathrm{C}$ until determination of the AAs by high-performance liquid chromatography (van Eijk et al., 1993). Total plasma TRP level and the TRP/ $L$ LNAA ratio were analyzed using repeated measures ANOVA with Time (t0 and t5) and Treatment (BAL or TRP-) as within-subject variables, and Order (TRP- or BAL mixture first) as between subject variable. Paired-sample t-tests were used to compare baseline measurements.

\section{Questionnaires}

Mood

A visual analogue version of the Profile of Mood States (POMS) was used to assess mood (McNair et al., 1988). This questionnaire consists of 32 bipolar sets of adjectives, which measure five mood dimensions: anger, depression, fatigue, tension and vigor. The items were scored on a 10-point scale. When the participants felt as they normally would, they were asked to mark the middle of the line (score 5).

\section{Adverse effect}

Adverse effects, 31 items, were registered and scored on a 4 points scale from 'no complaint at all' (0) to 'severe complaint' (4). A total score was calculated by adding the scores on the individual items.

The effect of ATD on mood and adverse effects was analyzed using repeated measures ANOVA with Time ( $\mathrm{t} 0$ and $\mathrm{t} 5$ ) and Treatment (BAL or TRP-) as withinsubject variables, and Order (TRP- or BAL mixture first) as between subject variable.

\section{Image acquisition}

Participants were scanned in a 1.5 tesla Philips scanner at the Academic Hospital Maastricht. T2*-weighted gradient echo planer images (EPI) (TE=27 ms) were acquired with BOLD contrast. A whole brain acquisition consisted of 24 slices (slice thickness $5 \mathrm{~mm}$; TR $1.75 \mathrm{sec}$; voxel size before normalization was 3.5 x 3.5 x $5 \mathrm{~mm}$ and after normalization $2 \times 2 \times 2 \mathrm{~mm}$; no slice gap; matrix size 64 x 64 x 24; oblique transversal orientation; flip angle $90^{\circ}$ ) and 190 volumes were acquired for each $\mathrm{Go} / \mathrm{NoGo}$ block. The stimulus presentation and the scanning were synchronized at the beginning of each run. High-resolution T1-weighted images for anatomical localization were made of each participant (voxel size 1 x 1 $\mathrm{x} 1 \mathrm{~mm})$. 


\section{Image analysis}

SPM2 (Statistical Parametric Mapping; Wellcome Department of Cognitive Neurology, London, UK) was used for data processing. Preprocessing procedures included slice acquisition time correction and within-subject realignment. Images from session 2 were then coregistered to the mean image from session 1 and thereafter spatially normalized to the standard Montreal Neurological Institute (MNI) structural template (SPM2). Finally, the images were spatially smoothed using a Gaussian ( $8 \mathrm{~mm}$ full-width at half maximum) kernel and high pass filtered.

A canonical hemodynamic response was used as a covariate in a general linear model and a parametric estimate was generated for each voxel for Gos, NoGos and Errors. Individual contrast images were taken to a second level analysis (one sample t-test), in which t-values were calculated for each voxel treating intersubject variability as a random effect. The hemodynamic response function was modeled to the onset of the response.

The following contrasts were calculated for the BAL and the TRP- condition together to assess task effects (whole brain analysis; Pcluster-corrected $<0.05$ ):

1. Performance monitoring: Errors minus Correct (NoGos + Gos) (contrast 1), (contrast weights -211 )

2. Response inhibition: NoGos minus Gos (contrast 2)

The following contrasts were calculated to assess the effect of ATD:

1. Effect of ATD on performance monitoring: contrast 1 in the TRPcondition compared with contrast 1 in the BAL condition (contrast 3 ).

2. Effect of ATD on response inhibition: contrast 2 in the TRP- condition compared with contrast 2 in the BAL condition (contrast 4).

3. As a control condition, effect of ATD on overall activation: all event types (Gos, NoGos and Errors) in the TRP- condition compared with all event types in the BAL condition (contrast 5).

The effect of ATD was analyzed using whole brain analysis (Pcluster-corrected< 0.05 and extent threshold of $>20$ voxels). In a previous study we showed that ATD increased the BOLD response in the dmPFC region after the last reversal error in a probabilistic reversal learning task (Evers et al., 2005). This region was used as a region of interest (ROI) (SPM, small volume correction; $10 \mathrm{~mm}$ sphere around the centre coordinated MNI: $x=9, y=39, z=48$ ) for the effect of ATD on brain activation during performance monitoring. The Talaraich Daemon was used to label the coordinates of the anatomical regions (http://ric.uthscsa.edu/TDapplet/). 


\section{Results}

Performance and fMRI data were complete for thirteen participants; eight participants started in the BAL condition and five started in the TRP-condition. Participants were scanned on average 5 hours after ingesting the AA mixture.

\section{Imaging}

No main task effect was found for performance monitoring (contrast 1; BAL and TRP- data together). However, in the BAL condition performance monitoring was associated with a significantly increased BOLD response in the ACC (MNI: $\mathrm{x}=6, \mathrm{y}=26, \mathrm{z}=40 ; \mathrm{T}=5.62$; Pcluster-corrected $=0.037 ; 156$ voxels; BA 32). Response inhibition (contrast 2; BAL and TRP- data together) was associated with a significantly increased BOLD response in a right inferior parietal/supramarginal cluster (BA 40), a right middle/superior frontal and precentral cluster (BA 10/44/46), a right medial/superior frontal cluster (BA 6/8), a left superior/inferior parietal and precuneus cluster (BA 7/40), a left inferior/middle frontal cluster (BA 8/9/47), and a right middle temporal cluster (BA 21) (see Table 1).

Table 1. Brain areas related to response inhibition; NoGo trials compared with Go trials for the BAL and the TRP- condition together. Only areas are shown for which Pcluster-corrected $<0.05$ in whole brain analysis.

\begin{tabular}{lccccc}
\hline & $\begin{array}{c}\text { MNI } \\
\text { coordinates }\end{array}$ & T-value & $\begin{array}{c}\text { P- } \\
\text { value }\end{array}$ & $\begin{array}{c}\text { Number } \\
\text { of voxels }\end{array}$ & $\begin{array}{c}\text { Brodmann } \\
\text { area }\end{array}$ \\
\hline Right inferior & $(48,-46,36)$ & 14.39 & 0.000 & 1666 & 40 \\
parietal/supramarginal & $(52,-52,42)$ & 12.09 & & & 40 \\
cluster & $(52,-54,30)$ & 10.01 & & & 40 \\
Right middle/superior & $(48,34,22)$ & 10.06 & 0.000 & 2357 & 46 \\
frontal and precentral & $(30,52,24)$ & 8.44 & & & 10 \\
cluster & $(52,10,6)$ & 7.85 & & & 44 \\
Right medial/superior & $(2,26,42)$ & 8.10 & 0.000 & 959 & 8 \\
frontal cluster & $(0,34,40)$ & 7.92 & & & 8 \\
& $(20,18,58)$ & 7.97 & & & 6 \\
Left superior/inferior & $(-34,-60,54)$ & 7.90 & 0.000 & 845 & 7 \\
parietal and & $(-58,-50,38)$ & 6.28 & & & 40 \\
precuneus cluster & $(-28,-72,50)$ & 5.97 & & & 47 \\
Left inferior/middle & $(-30,26,-6)$ & 7.12 & 0.000 & 639 & 8 \\
frontal cluster & $(-48,10,42)$ & 5.86 & & & 9 \\
& $(-40,30,28)$ & 5.36 & & & 21 \\
Right middle temporal & $(60,-36,-6)$ & 6.06 & 0.045 & 122 & 21 \\
cluster & $(60,-26,-12)$ & 5.89 & & & \\
\hline
\end{tabular}


ATD decreased the BOLD response in the dmPFC cortex (ROI analysis; MNI: $\mathrm{x}=4, \mathrm{y}=44, \mathrm{z}=40 ; \mathrm{T}=4.98 ;$ Pcluster-corrected $=0.037 ; 7$ voxels; BA 8) during performance monitoring (contrast 3). No effect of ATD was found on brain activation associated with response inhibition (contrast 4). Percent signal change for both the BAL and the TRP- condition in the dmPFC activation cluster is shown in figure 1 .

Since no effect of ATD on overall activation was found (contrast 5), the effect of ATD in the dmPFC was not confounded by overall effects of ATD. Talbot and Cooper (in press) showed that individual changes in depressed mood after ATD, without an overall mood effect, affected the BOLD response in the subgenual ACC and associated regions. Therefore we conducted a post hoc analysis to examine whether the effect of ATD on the BOLD response in the dmPFC is confounded by mood changes. Post hoc ANCOVA analysis showed that the effect of ATD on the response in the dmPFC was not confounded by mood. After correction for the delta depression score (score on the depression subscale of the POMS at TRP- [t5 - $\mathrm{t} 0$ ] minus BAL [t5 - t0]) ATD decreased the BOLD response in the dmPFC (ROI analysis; MNI: $\mathrm{x}=4, \mathrm{y}=44, \mathrm{z}=40$; $\mathrm{T}=4.84$; Pclustercorrected $=0.048 ; 4$ voxels; BA 8). In addition, the effect of ATD during performance monitoring was not confounded by the fact that eight participants started in the BAL and five participants in the TRP- condition. Repeated measures analysis with Treatment as within subject factor and Order as between subject factor showed that the percent signal change extracted from the dmPFC (two times Error minus Go and minus NoGo percentage signal change) was not affected by Order (Treatment $x$ Order interaction: $F(1,11)=3.6 ; \mathrm{p}=0.08$ ).

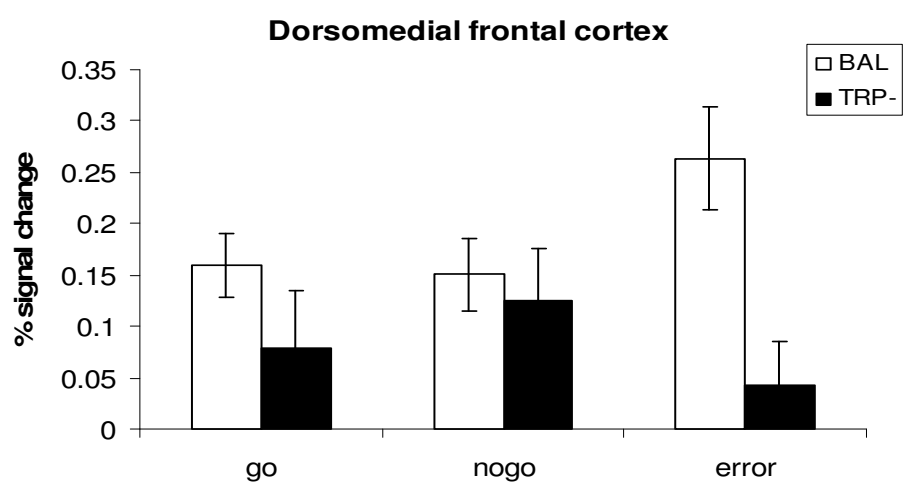

Figure 1. Percent signal change in the BAL and the TRP- condition for the dorsomedial prefrontal activation cluster. Percent signal changes were calculated with Marsbar (http://marsbar.sourceforge.net/). The presented percentage signal change is the mean value for the two blocks per condition. 


\section{Biochemical results}

In the BAL condition blood samples from twelve participants and in the TRPcondition blood samples from thirteen participants were available for analysis. No difference in total plasma TRP (mean/SEM: BAL 47.6/1.5; TRP- 47.4/1.3) and the TRP/ $\Sigma$ LNAA ratio (BAL 0.10/0.01; TRP 0.10/0.00) at baseline (t0) was found between the BAL and the TRP-condition. In the TRP- condition total plasma TRP $(19.4 / 2.5)$ and the TRP/ $\Sigma$ LNAA ratio $(0.02 / 0.00)$ were significantly lowered (Treatment $*$ Time interaction for total plasma TRP: $\mathrm{F}(1,10)=44.7 ; \mathrm{p}=0.000$; Treatment $*$ Time interaction for the ratio: $\mathrm{F}(1,10)=96.9 ; \mathrm{p}=0.000)$ five hours after the AA mixture. Total plasma TRP dropped by $59 \%$ and the TRP/ $\Sigma$ LNAA ratio by $77 \%$ after ingesting the TRP- mixture. In the BAL condition levels of total plasma TRP (108.5/12.2) were significantly increased ( $\mathrm{t} 11=-4.9 ; \mathrm{p}<0.01)$, but the TRP/ $/$ LNAA ratio $(0.11 / 0.01)$ was not changed $(\mathrm{T} 11=-1.15 ; \mathrm{p}>0.05)$ five hours after the AA mixture. Total plasma TRP increased by $128 \%$ and the TRP/ $\Sigma$ LNAA ratio increased by $15 \%$ after the BAL mixture.

\section{Performance}

Performance data $(n=13)$ is shown in Table 2. No effect of ATD was found on the number of correct Gos and correct NoGos. Participants successfully completed on average 18.6 correct NoGos $(\mathrm{SE}=1.2)$ in the TRP- condition and 17.1 correct NoGos $(\mathrm{SE}=1.5)$ in the BAL condition. Participants made on average 17 Errors $(\mathrm{SE}=3.5)$ in the TRP- condition and 21 Errors $(\mathrm{SE}=4.3)$ in the BAL condition. In the BAL and in the TRP- condition participants reacted fasted on a correct Go trial after a correct response than on a correct Go trial after an incorrect Go trial (BAL: $\mathrm{T}=-4.77 ; \mathrm{p}=0.000$, TRP-: $\mathrm{T}=-3.5 ; \mathrm{p}=0.001)$ and after an incorrect NoGo trial (BAL: $\mathrm{T}=-2.0 ; \mathrm{p}=0.04$, TRP-: $\mathrm{T}=-3.3 ; \mathrm{p}=0.002$ ). No effect of ATD was found on the RT measures.

Table 2. Performance data for the Go/NoGo task: means with standard errors of the mean and the total number of events for reaction times.

\begin{tabular}{lcc}
\hline Events & BAL & TRP- \\
\hline Percentage correct NoGos & $68(2)$ & $72(1)$ \\
Percentage correct Gos & $91(3)$ & $93(3)$ \\
$\begin{array}{l}\text { Mean reaction time (ms) on a correct Go after a } \\
\text { correct response }\end{array}$ & $370(1)$ & $369(1)$ \\
Mean reaction time (ms) on a correct Go after an & $375(5)$ & $(\mathrm{n}=1738)$ \\
error on a NoGo trial & $(\mathrm{n}=92)$ & $373(6)$ \\
$\begin{array}{l}\text { Mean reaction time (ms) on a correct Go after an } \\
\text { error on a Go trail (misses) }\end{array}$ & $384(4)$ & $379(6)$ \\
& $(\mathrm{n}=141)$ & $(\mathrm{n}=110)$ \\
\hline
\end{tabular}




\section{Subjective measures}

Data from the POMS questionnaire, which was complete for all thirteen participants, showed that no effect of ATD was found on the subscales for depression $(\mathrm{F}(1,11)=0.1 ; \mathrm{P}=\mathrm{NS})$, anger $(\mathrm{F}(1,11)=0.2 ; \mathrm{P}=\mathrm{NS})$, fatigue $(\mathrm{F}(1,11)=0.7$; $\mathrm{P}=\mathrm{NS})$, vigor $(\mathrm{F}(1,11)=0.1 ; \mathrm{P}=\mathrm{NS})$ and tension $(\mathrm{F}(1,11)=0.0 ; \mathrm{P}=\mathrm{NS})$. A significant main effect of Time was found for vigor $(\mathrm{F}(1,11)=9.8 ; \mathrm{P}<0.05)$. At t0 the participants felt more vigorous (total score of five vigor items is 207; $\mathrm{SE}=17.6$ ) than at t5 (total score of five vigor items is 224; $\mathrm{SE}=13.2$ ).

Data of one subject were missing from the physical complaints list $(n=12)$. No effect of ATD on physical complaints was found $(\mathrm{F}(1,10)=1.8 ; \mathrm{P}=\mathrm{NS})$. In the BAL condition the participants had on average a total score of $3.3(\mathrm{SE}=0.9)$ at t0 and 4.2 $(\mathrm{SE}=1.0)$ at $\mathrm{t5}$. In the TRP- condition the participants had on average a total score of $2.2(\mathrm{SE}=0.8)$ at $\mathrm{t} 0$ and $3.6(\mathrm{SE}=1.0)$ at t5.

\section{Discussion}

The present study investigated the effect of a transient lowering of 5-HT on performance monitoring and response inhibition related brain activation in thirteen healthy male volunteers. In line with previous studies (Asahi et al., 2004; Durston et al., 2002; Garavan et al., 1999, 2002, 2003; Horn et al., 2003; Kelly et al., 2004; Liddle et al., 2001; Menon et al., 2001; Rubia et al., 2003), the present study showed that a widespread network of associated brain regions is involved in response inhibition, including areas in the frontal, parietal and the temporal cortex. ATD successfully decreased plasma TRP (-59\%) and the TRP/LLNAA ratio ($77 \%$ ), but did not affect mood and task performance. ATD decreased the BOLD response in the dmPFC (BA 8) during performance monitoring and did not change the BOLD response during response inhibition.

\section{Performance monitoring}

The present study revealed that ATD modulated the BOLD response in the dmPFC during performance monitoring in a Go/NoGo paradigm in healthy volunteers. The dmPFC has been associated with cognitive flexibility. Previous studies have shown that the dmPFC (BA 8) is involved in switching behavior during a reversal learning task (Cools et al., 2002; Kringelbach and Rolls, 2003; Remijnse et al., 2005) and response uncertainty during a decision making task (Volz et al., 2004, 2005). Moreover, the medial prefrontal cortex is strongly innervated by the 5-HT projections from the rostral raphe nuclei (for review see Hornung 2003). In line with these findings, a previous pharmacological neuroimaging study (Evers et al., 2005) reported that ATD modulates the BOLD response in the DMPFC after a final reversal error in a probabilistic reversal 
learning task in healthy volunteers. These data suggest a role for 5-HT in the functioning of the dmPFC during cognitive flexibility tasks.

To examine the effect of ATD on performance monitoring, we calculated the mean reaction time on the correct response after an error (see Table 2). It is a welldocumented finding that participants slow down after an error: post error slowing (first postulated by Rabbitt 1966). It is noteworthy that although ATD changed the BOLD response in the dmPFC during performance monitoring, we did not find reaction time changes on a correct trial after negative feedback. The performance measures used in this study might not have been sensitive enough to pick up subtle behavioral changes associated with the changed brain activation. Several previous ATD studies also failed to find behavioral effects accompanying the reported effects of ATD on brain activation (Evers et al., 2005; Horacek et al., 2005; Rubia et al., 2005).

In contrast with the present study, which showed that ATD decreased the BOLD response in the DMPFC during performance monitoring, our research group (Evers et al., 2005) showed in a previous study that ATD increased the BOLD response in the DMPFC after a last reversal error in a probabilistic reversal-learning task. This opposite effect might be related to the different meaning of the negative feedback in the reversal learning and the Go/NoGo task. In the probabilistic reversal learning task negative feedback after a last reversal error signaled a response error due to a change of the stimulus-response rule, whereas in the Go/NoGo task negative feedback signaled a response error due to suboptimal performance. Clearly, this hypothesis needs to be tested by future research. Aside from task-related differences, there might be another explanation for the contradictory findings. Since ATD studies have shown contradictory findings in the past (for example Rogers et al., 1999b versus Talbot et al., 2006), Talbot et al. (2006) hypothesized that personality characteristics play a decisive role in the effect of ATD on performance and brain activation. Support for this hypothesis is provided by behavioral ATD studies that showed that ATD only affects sensitive individuals. For example, ATD lowers mood in individuals with a positive family history of major depression (see review Riedel et al., 2002) and in individuals that are susceptible to lowered mood or aggression (see review Young and Leyton, 2002). Moreover, Cools et al. (2005b) showed that ATD modulates the amygdala activation in response to fearful faces as a function of self-reported threat sensitivity, and that ATD impaired motivational guidance of goal-directed behavior on a cued-reinforcement reaction time task as a function of the trait impulsivity (Cools et al., 2005a). Since in our previous and present study participants were not screened and selected on the basis of personality traits, it is possible that unknown personality differences influenced the results. Clearly, this 
hypothesis needs to be tested by future research. The present study suggests that individual mood changes do not account for these inconsistencies.

Fallgatter et al. (2004) reported that carriers of the short allele variant of a functional length variation in the transcriptional control region of the 5-HT transporter gene, which has been associated with an increased risk for depression and anxiety (for review see Hariri et al., 2006), showed higher amplitude of the error-related negativity compared to carriers of two long alleles. This indicates that a short allele is associated with an enhanced responsiveness of the anterior cingulate cortex (ACC) during error processing. The present study did not show an effect of low 5-HT on ACC activation, but did show decreased activation in the dmPFC after ATD during performance monitoring. The decreased dmPFC activation did not overlap with the task related ACC activation in the BAL condition during performance monitoring. Differences in the experimental design and the 5-HT manipulation might explain the differential effect of low 5-HT on brain activation in these studies. Future studies directly examining the effects of ATD on the ERN might increase our insight in the effect of low 5-HT in dorsomedial frontal regions during performance monitoring.

\section{Response inhibition}

The present study did not show that ATD modulated the BOLD response in the right inferior frontal cortex, as the study by Rubia et al. (2005) reported. This might be due to task and design related differences in the Go/NoGo tasks that were used. Firstly, the present study used a more difficult Go/NoGo task. The participant had to respond faster due to a predefined time range and instead of different symbols the same symbols were used for Gos and NoGos. Due to these differences participants responded faster ( $375 \mathrm{~ms}$ versus $459 \mathrm{~ms}$ ) and made more errors (70\% versus $98 \%$ of the NoGo trials were successful) in the present study as compared with Rubia's study. Secondly, performance feedback was given during the task used in the present study. Thirdly, in the study of Rubia et al. both females and males were tested, whilst in our study only males were used. Previous studies showed that females respond differently to ATD than males do. Booij et al. (2002) showed that total and free plasma TRP decreased more in females then in males after ATD. Harmer et al. (2003) showed that tryptophan depletion decreased recognition of fear in female, but not in male, volunteers. At the moment it remains unclear to what extent the effect of ATD on the BOLD response during cognitive flexibility tasks differs between males and females.

The lack of effect of ATD on performance is in agreement with studies that did not find behavioral effects of ATD on response inhibition (Clark et al., 2005; Cools et al., 2005a, Le Marquand et al., 1998, 1999; Rubia et al., 2005). These 
studies used a Go/NoGo task or a Stop Signal task to measure response inhibition. In contrast, a study by Walderhaug et al. (2002) reported that ATD increased an impulsive response style on a continuous performance task (CPT) in healthy volunteers. An increase in impulsive response style on the CPT indicates that subjects responded more often to correct stimuli and to catch trials. Because impaired response inhibition on a Go/NoGo or a Stop Signal Task and an impulsive response style on a CPT task reflect a different impairment, it is possible that ATD affects these measures differently.

\section{Conclusions}

The present study showed that ATD modulated the BOLD response during performance monitoring, while leaving performance unchanged. This is in agreement with other studies that showed that 5-HT is implicated in cognitive flexibility. Since ATD studies have revealed inconsistent effects of ATD on performance and on brain activation, it was hypothesized that the effect of ATD might depend on gender and personality characteristics. Clearly, more research is needed to unravel the effects of low 5-HT on cognitive flexibility. 


\title{
Acute tryptophan depletion improves performance and modulates the BOLD response during a Stroop task in healthy females
}

\author{
EAT Evers, FM van der Veen, J Jolles, NEP Deutz, JAJ Schmitt
}

\begin{abstract}
To gain more insight into the effect of low brain serotonin (5-HT) on brain activation related to conflict, the present study examined the effect of acute tryptophan depletion (ATD), on performance and the blood oxygen level dependent (BOLD) response during a combined cognitive and emotional Stroop task. Fifteen healthy female volunteers were tested during a placebo and tryptophan depletion session in an event-related fMRI design. ATD improved performance during Stroop interference. Two effects of ATD on the BOLD response were found. Firstly, ATD increased the BOLD response in the anterior cingulate cortex (ACC) (BA 32) when incongruent colour words were compared with congruent colour words in the first Stroop block the participants performed. Secondly, ATD increased the BOLD response in the left precuneus (BA 31) and cuneus (BA 18) during congruent colour words. ATD did not affect the BOLD response accompanying emotional stimuli. However, we showed that ATD increased the interference of negative words on colour naming. This finding was explained in terms of an emotional processing bias in favour of negative words, which leads to stronger interference of these words. In line with previous studies the present study showed that a temporary reduction of 5-HT improved Stroop performance and changed the underlying brain activation pattern in healthy female participants. Moreover, we replicated our previous finding that ATD modulated the BOLD response in the dorsomedial prefrontal cortex during tasks that require cognitive control.
\end{abstract}

\section{Acknowledgements}

We thank Olga Reneerkens for test and analysis assistance and Jeroen van Deursen for radiographic assistance. This work was supported by a TOP grant (No. 912-02-050) from ZonMW-NWO and a grant from the Dutch Brain Foundation (Hersenstichting Nederland, 11F03(2).41)

In press in Neuroimage 


\section{Introduction}

Acute tryptophan depletion (ATD) has been used as a model to study the effects of reduced central 5-HT (Nishizawa et al., 1997; Williams et al., 1999; Young et al., 1999) on cognitive performance and brain activation. In a previous study our group showed that ATD increased the response in the dorsomedial prefrontal cortex (dmPFC) to negative feedback preceding a switch in response strategy (Evers et al., 2005). Activation in the dmPFC associated with negative feedback has been related to performance monitoring and cognitive control (see Ridderinkhof $2004 \mathrm{~b}$ for a review). According to one theory (Holroyd and Coles, 2002) the dmPFC response to negative feedback is linked to phasic changes in the midbrain dopamine system related to outcomes that are worse than expected. According to a second theory dmPFC activation is related to conflict monitoring which becomes necessary when two competing response tendencies become active at the same time (Botvinick et al., 2004). In our previous study (Evers et al., 2005) it was unclear whether the dmPFC response to negative feedback was associated with an outcome that was worse than expected or conflict monitoring. Therefore, the present study examined brain responses to conflict in the absence of negative outcome. This was done in a combined cognitive and emotional Stroop task.

The studies examining the effects of ATD on performance in a Stroop task have been inconclusive thus far. Some studies reported improved performance (Coull et al., 1995; Rosse et al., 1992; Rowley et al., 1997; Schmitt et al., 2000), whereas other studies did not show an effect of ATD on Stroop performance (Gallagher et al., 2003; Horacek et al., 2005; Sobczak et al., 2002). Horacek et al. (2005) showed that ATD increased the Blood Oxygen Level Dependent (BOLD) response in the left bilateral mediofrontal, anterior cingulate and dorsolateral prefrontal cortex during Stroop performance in healthy volunteers. A problem with this study is that performance was measured outside the MRI scanner. It is therefore not possible to judge whether the participants carried out the task correctly during scanning. The current study used an event-related design to study the effect of ATD on the BOLD response during Stroop interference. Performance and the BOLD signal were recorded simultaneously.

5-HT has also been related to emotional processing. Previous studies revealed that ATD impaired the processing of positive information in healthy volunteers (Murphy et al., 2002) and remitted depressed patients (Booij et al., 2005). However, Booij et al. (2005) showed no effect of ATD on reaction times (RTs) and interference scores during an emotional Stroop task in currently depressed patients. In general, participants experience more interference from negative than 
from positive or neutral words (e.g. McKenna and Sharma, 1995) which can be explained by the higher threat caused by negative events in daily live (Mandler 1975; Oatley and Johnson-Laird, 1987). Compton et al. (2003) showed that ignoring negative versus neutral words activated the bilateral occipito-temporal cortex and decreased the activation in the amygdala. Whalen et al. (1998) reported greater activation in the anterior cingulate cortex (ACC) for negative versus neutral words. However, Compton et al. (2003) did not find brain activation differences between positive and neutral words. To our knowledge, the present study is the first study examining the effect of ATD on the BOLD response during an emotional Stroop task.

To study the effect of ATD on cognitive Stroop interference and interference by emotional words, we designed a combined Stroop task in which congruent colour (CC), incongruent colour (IC), neutral (e.g. house, bean, coin), positive (e.g. proud, friend, smart) and negative (e.g. murder, bitch, death) words were presented in a semi-randomized order. Based on the studies discussed above, we hypothesized that (i) IC words cause more interference than CC words, (ii) IC words are associated with an increased BOLD response in the ACC, inferior parietal cortex, inferior frontal junction and middle frontal cortex (Laird et al., 2005) and (iii) ATD does not change or decreases Stroop interference and modulates the related BOLD response. With regard to the emotional Stroop part we hypothesized that (i) RTs are higher for negative than for positive or neutral words, because of the higher threat caused by negative events, (ii) the medial PFC, ACC and insula are activated during emotional words (for review see Phan et al., 2002) and (iii) ATD impairs performance on emotional words and affects the related brain activation.

\section{Materials and methods}

\section{Participants}

Nineteen healthy female volunteers (aged between 19 and 33; mean age 22.3; SE of mean 0.7), mostly pregraduate students, were included in this study, which was approved by the Medical Ethics Committee of Maastricht University Hospital. Participants were recruited by local advertisements. The health status of the participants were checked by a medical questionnaire, which was evaluated by a medical doctor. The participants received no medication at the moment of inclusion, had never used anti-depressants or ecstasy, did not have a family history of affective disorder and were screened for MRI contra-indications. All participants gave written informed consent, and were paid 75 euros. 


\section{Experimental design}

Participants were scanned at the Maastricht University Hospital radiology department on two separate test days in an experimental and a placebo test session, counterbalanced according to a double-blind crossover design. Participants arrived in the morning, after they had fasted overnight and eaten a low protein breakfast, and were given a tryptophan-depleted (TRP-) or a balanced (BAL) amino acid (AA) mixture, followed by a four and a half hour break. During this break only low protein food and protein and caffeine free drinks were consumed. After the break (between 2 and 8.30 p.m.) the participants were scanned while performing two blocks of an emotional Stroop task, each lasting about 9 minutes. Halfway through the scanning session a structural scan was made, which lasted for about 10 minutes. Before the first test session participants were trained in a dummy scanner.

\section{Stroop task}

The participants were instructed to report the colour of the ink in which the presented words were printed by pressing one of four response buttons. Two fiberoptic response devices with two buttons were used. On the first response box, the left middle finger was used for blue, the left index finger for red. On the second response box, the right index finger was used for green, and the right middle finger for yellow. This colour-response correspondence was written in small white letters on the bottom of the screen, along with each presented word. The participants were instructed to learn this correspondence by heart, and to look at the bottom of the screen only if their memory failed. The task was programmed in E-Prime V1.0 (Psychology Software Tools, 2002). A word was presented every 2 seconds against a black background, and stayed on the screen until a response was given. A black screen was shown between the response and the next stimulus. No feedback was given.

Participants completed two blocks of the modified Stroop task in each test session. Each block contained 40 CC, 40 IC, 24 positive, 24 negative and 24 neutral words, which were presented in a semi-randomized order (never the same colour three times in a row). Each word type was presented equally often in each of the four colours. A test block started with 10 neutral words that were not included in the analysis. During the training session the participants performed two Stroop blocks each containing 40 CC, 40 IC and 72 neutral words.

The following performance measures were used: mean RT and the number of errors for IC, CC, neutral, positive and negative words, and interference scores. Interference scores for the cognitive Stroop express the extra time needed for IC compared with CC words ((RT for IC words - RT for CC words)/ RT for CC words) as a percentage. As regards the emotional Stroop, interference scores were calculated for positive compared with neutral words, and for negative compared with neutral words (Booij et al., 2005). 


\section{Acute tryptophan depletion}

The present study used ATD to temporary lower 5-HT in the human brain (Young et al., 1985). The TRP- mixture $(75 \mathrm{~g}$ ) contained $4.1 \mathrm{~g}$ L-alanine, $2.4 \mathrm{~g} \mathrm{~L}$ glycine, $2.4 \mathrm{~g}$ L-histidine, $6.0 \mathrm{~g}$ L-isoleucine, $10.1 \mathrm{~g} \mathrm{~L}$-leucine, $6.7 \mathrm{~g} \mathrm{~L}$-lysine, 4.3 g L-phenylalanine, $9.2 \mathrm{~g}$ L-proline, $5.2 \mathrm{~g} \mathrm{~L}$-serine, $4.9 \mathrm{~g}$ L-threonine, $5.2 \mathrm{~g} \mathrm{~L}$ tyrosine, $6.7 \mathrm{~g} \mathrm{~L}$-valine, $3.7 \mathrm{~g} \mathrm{~L}$-arginine, $2.0 \mathrm{~g}$ L-cysteine, $2.3 \mathrm{~g}$ L-methionine. In the BAL mixture $3.0 \mathrm{~g}$ TRP was added. The mixtures were prepared with $200 \mathrm{ml}$ tap water.

\section{Biochemical measures}

Blood samples $(10 \mathrm{ml})$ were taken before ingestion of the AA mixture and about fifteen minutes before the start of the scanning session to determine the plasma TRP level and the TRP/ $/$ LNAA ratio ([TRP] / [tyrosine + leucine + phenylalanine + isoleucine + valine]). Blood samples were immediately centrifuged at $4^{\circ} \mathrm{C}(10$ minutes, $4500 \mathrm{rpm})$. Hundred $\mu$ l aliquot of plasma was mixed with $8 \mathrm{mg}$ sulfasalicyl acid and frozen at $-80{ }^{\circ} \mathrm{C}$ until determination of the AAs by high-performance liquid chromatography (van Eijk 1993).

\section{Questionnaires \\ Mood}

A short visual analogue version of the Profile of Mood States (POMS) was used to assess mood (McNair et al., 1988). This questionnaire consists of 32 bipolar sets of adjectives, which measure five mood dimensions: anger, depression, fatigue, tension and vigour. The items were scored on a 10-point scale.

\section{Adverse effects}

Adverse effects, 31 items, were registered and scored on a 4 points scale from 'no complaint at all' (0) to 'severe complaint' (4). A total score was calculated by adding the scores on the individual items.

\section{Statistical analysis}

The effect of ATD on the RTs and the number of errors were analyzed (SPSS version 11.5 for Windows) using GLM repeated measurements with Trial Type (IC and CC, or neutral, positive and negative words) and Treatment (BAL or TRP) as within subject factors and Order (TRP- or BAL mixture first) as betweensubject factor. The effect of ATD on interference scores was analyzed using GLM repeated measures with Treat as within subject variable and Order as between subject variable. Plasma TRP level and the TRP/LLNAA ratio were analyzed using GLM repeated measurements with Time ( $\mathrm{t} 0$ and $\mathrm{t} 5$ ) and Treatment as within subject variables, and Order as between subject variable (Greenhouse-Geisser correction). Paired-sample t-tests were used to compare baseline measurements. 
The effect of ATD on mood and adverse effects was analyzed using GLM repeated measurements with Time and Treatment as within subject variables, and Order as between subject variable (Greenhouse-Geisser correction).

\section{Image acquisition}

Participants were scanned in a 1.5 tesla Philips scanner at the Maastricht University Hospital. T2*-weighted gradient echo planer images (EPI) (TE=27 ms) were acquired with BOLD contrasts. A whole brain acquisition consisted of 24 slices (slice thickness $5 \mathrm{~mm}$; $\mathrm{TR}=1.75 \mathrm{sec}$; voxel size before normalization was $3.5 \times 3.5 \times 5 \mathrm{~mm}$ and after normalization $2 \times 2 \times 2 \mathrm{~mm}$; no slice gap; matrix size 64 x 64 × 24; oblique transversal orientation; flip angle $90^{\circ}$ ) and 220 volumes were acquired for each Stroop block. The stimulus presentation and the scanning were synchronized at the beginning of each run. High-resolution T1-weighted images for anatomical localization were made of each participant (voxel size $1 \times 1 \times 1$ $\mathrm{mm})$.

\section{Image analysis}

SPM2 (Wellcome Department of Cognitive Neurology, London, UK) was used for data processing. Preprocessing procedures included slice acquisition time correction (slice 12 as reference slice; $\mathrm{TA}=1.68$ ) and within subject realignment (Realign and Unwrap) using the first slice as a reference. Images from session 2 were then coregistered to the mean image from session 1 and thereafter spatially normalized to the standard Montreal Neurological Institute (MNI) structural template (average of $152 \mathrm{~T} 1$ brains). Finally, the images were spatially smoothed using a Gaussian ( $8 \mathrm{~mm}$ full-width at half maximum) kernel and high pass filtered (128 s). A simple hemodynamic response was used as a covariate in a general linear model and a parametric estimate was generated for each voxel for each trial type (correct and incorrect). Individual contrast images were taken to a second level analysis, in which t- values were calculated for each voxel treating intersubject variability as a random effect. The hemodynamic response function was modelled to the onset of the response.

The following contrasts were calculated to assess task effects (BAL data only): 1) IC words versus all other trial types, 2) $\mathrm{CC}$ words versus all other trial types, 3) IC minus CC words, 4) neutral words versus all other trial types, 5) positive words versus all other trial types, 6) negative words versus all other trial types, 7) positive minus neutral words, 8) negative minus neutral words. These contrasts were analyzed using whole brain analysis (Pcluster-corrected $<0.05$ ).

The following contrasts were calculated to assess the effect of ATD: 9) IC words in the TRP- compared with IC words the BAL condition (IC words $\mathrm{x}$ ATD), 10) $C C$ words $x$ ATD, 11) [IC minus $C C$ words] $x$ ATD, 12) neutral words $x$ ATD , 13) positive words $x$ ATD, 14) negative words $x$ ATD, 15) [positive minus 
neutral words] x ATD, 16) [negative minus neutral words] x ATD, 17) all events (IC, CC, neutral, positive and negative words) x ATD.

The effect of ATD was analyzed using whole brain analysis (Pcluster-corrected < 0.05). ROI analyses were executed for the IC words (contrasts 9 and 11) using the following ROIs: brain areas that showed increased activation after ATD during a Stroop task as revealed by Horacek et al. (2005): the left inferior frontal gyrus (BA 47; TAL: $\mathrm{x}=-24, \mathrm{y}=12, \mathrm{z}=-16$ ) and the right medial frontal gyrus (BA 10; TAL: $\mathrm{x}=6, \mathrm{z}=52, \mathrm{z}=0$ ). We use WFU-PickAtlas tool (Maldjian et al., 2003) for ROI analysis. Ten mm spheres were built around the centre coordinates. The Talaraich Daemon was used to label the coordinates of the anatomical regions (http://ric.uthscsa.edu/TDapplet/).

\section{Results}

Fifteen volunteers were successfully tested. Of the original 19 included volunteers, two dropped out after the first session because of nausea and vomiting, one volunteer panicked in the scanner, and imaging data for one participant were lost due to technical problems. Nine participants started in the BAL condition and six started in the TRP- condition.

\section{Biochemical results}

Blood samples were not complete for five participants due to technical problems. Data are presented for ten participants. ATD lowered $(F(1,8)=24.8$; $\mathrm{p}=0.001)$ the TRP plasma level by $80 \%$. Mean TRP level on the BAL condition was $43.7 \mu \mathrm{M}(\mathrm{SE}=1.8)$ at $\mathrm{t} 0$ and $132.0 \mu \mathrm{M}(\mathrm{SE}=13.2)$ at t5. Mean TRP levels in the TRP- condition were $46.0 \mu \mathrm{M}(\mathrm{SE}=3.1)$ at $\mathrm{t} 0$ and $9.2 \mu \mathrm{M}(\mathrm{SE}=0.7)$ at $\mathrm{t} 5$. No significant difference in plasma TRP $(\mathrm{T}=-1.1 ; \mathrm{p}=0.3)$ was present at baseline $(\mathrm{t} 0)$ between the BAL and the TRP-condition. ATD lowered $(F(1,8)=13.6 ; p<0.01)$ the TRP $/ \Sigma L N A A$ ratio by $91 \%$. The mean ratio in the BAL condition was 0.12 $(\mathrm{SE}=0.01)$ at $\mathrm{t} 0$ and $0.14(\mathrm{SE}=0.01)$ at $\mathrm{t} 5$. The mean ratio in the TRP- condition was $0.12(\mathrm{SE}=0.01)$ at $\mathrm{t} 0$ and $0.01(\mathrm{SE}=0.01)$ at $\mathrm{t} 5$. No significant difference $(\mathrm{T}=0.9 ; \mathrm{p}=0.4)$ in the ratio was present at baseline between the BAL and the TRPcondition.

\section{Performance}

Cognitive Stroop. RTs and percentage incorrect responses for the different event types are presented in Table 1. GLM analysis showed a main effect of Trial Type for RT $(\mathrm{F}(1,13)=61.2 ; \mathrm{p}<.001)$ : participants responded slower on IC than on $\mathrm{CC}$ words. No main effect of ATD was found on RTs $(F(1,13)=1.2 ; p=0.30)$. 
Statistical analysis did not show an effect of ATD on the number of error on IC and CC words. GLM analyses revealed an effect of ATD on the interference score for IC words $(\mathrm{F}(1,13)=4.5 ; \mathrm{p}=0.057)$. Because of our a priori hypothesis that ATD does not change or decrease Stroop interference it is allowed to test this hypothesis one sided. We therefore concluded that ATD significantly decreased $(\mathrm{F}(1,13)=4.5$; $\mathrm{p}=0.03)$ interference for IC words compared with $\mathrm{CC}$ words. This effect was not confounded by the order of testing $(\mathrm{F}(1,13)=0.4 ; \mathrm{p}=0.5)$.

Table 1. Performance data: mean reaction times $(\mathrm{ms})$, the percentage incorrect responses per word type and the interference scores, with errors of the mean

\begin{tabular}{lccc}
\hline & Event type & BAL condition & TRP- condition \\
\hline Mean reaction time & & \\
incon & $768(24)$ & $736(20)$ \\
con & $650(16)$ & $636(16)$ \\
neu & $705(29)$ & $694(25)$ \\
pos & $687(26)$ & $698(26)$ \\
neg & $717(28)$ & $705(30)$ \\
Interference (\%) & & \\
& & $19(2)$ & $13(2)$ \\
Percentage errors & incon versus con & $-2.3(1)$ & $0.5(1)$ \\
& pos versus neu & $1.9(1)$ & $1.4(1)$ \\
neg versus neu & & \\
& & $7.5(0.6)$ & $9.0(0.8)$ \\
& incon & $3.3(0.4)$ & $4.2(0.5)$ \\
& con & $4.8(0.4)$ & $3.0(0.3)$ \\
& neu & $4.8(0.3)$ & $4.8(0.4)$ \\
& pos & $3.8(0.3)$ & $8.3(0.4)$ \\
\hline
\end{tabular}

Emotional Stroop. RTs and percentage incorrect responses for the different event types are presented in Table 1. GLM analysis showed a main effect of Emotion on RT $(\mathrm{F}(1,13)=3.9 ; \mathrm{p}<.05)$. Paired T-tests showed that participants reacted slower to negative $(711 \mathrm{~ms}, \mathrm{SE}=26)$ than to positive $(693 \mathrm{~ms}, \mathrm{SE}=25$; $\mathrm{T}=2.2 ; \mathrm{p}<.05$ ), and neutral words $(700 \mathrm{~ms}, \mathrm{SE}=28 ; \mathrm{T}=2.6 ; \mathrm{p}<.05)$. RTs for positive and neutral words did not differ. No effect of ATD on RTs was found. GLM analysis showed a significant interaction between Treatment and Emotion $(\mathrm{F}(2,26)=9.8 ; \mathrm{p}<.01)$ for the number of errors. Paired T-tests showed that the numbers of errors on negative words increased after ATD $(\mathrm{T}=3.2 ; \mathrm{p}<.01)$. No effect of ATD on the interference scores was found. 
Table 2. Brain areas activated during the different words types in the BAL condition revealed by whole brain analysis. $\mathrm{CC}=$ congruent colour words, $\mathrm{IC}=$ incongruent colour words, $\mathrm{BA}=$ Brodmann area

\begin{tabular}{|c|c|c|c|c|c|c|}
\hline & $\begin{array}{l}\text { Hemi } \\
\text { sphere }\end{array}$ & $\begin{array}{c}\text { MNI } \\
\text { coordinates }\end{array}$ & T-value & $\begin{array}{l}\text { Pcluster- } \\
\text { correctd }\end{array}$ & $\begin{array}{l}\text { Number } \\
\text { of voxels }\end{array}$ & $\mathrm{BA}$ \\
\hline \multicolumn{7}{|l|}{ IC words } \\
\hline Inferior parietal cortex & left & $(-50,-34,48)$ & 6.23 & 0.021 & 190 & 40 \\
\hline Middle frontal cortex & left & $(-34,32,34)$ & 5.80 & 0.001 & 324 & 9 \\
\hline $\begin{array}{l}\text { Superior temporal } \\
\text { cortex }\end{array}$ & right & $(52,14,-4)$ & 4.97 & 0.027 & 179 & 22 \\
\hline \multicolumn{7}{|l|}{ CC words } \\
\hline Inferior parietal cortex & left & $(-52,-34,48)$ & 6.44 & 0.012 & 212 & 40 \\
\hline Inferior frontal cortex & right & $(58,20,2)$ & 5.14 & 0.046 & 152 & 45 \\
\hline \multicolumn{7}{|l|}{ IC minus CC words } \\
\hline Inferior frontal cortex & left & $(-40,24,26)$ & 6.89 & 0.002 & 279 & 45 \\
\hline & left & $(-38,10,22)$ & 5.62 & & & 44 \\
\hline \multicolumn{7}{|c|}{ IC minus CC words first Stroop block } \\
\hline $\mathrm{ACC}$ & Bil. & $(0,36,22)$ & 4.83 & 0.044 & 27 & 32 \\
\hline Positive words & & & & & & \\
\hline $\begin{array}{l}\text { Inferior temporal } \\
\text { cortex }\end{array}$ & left & $(-46,-26,-22)$ & 5.72 & 0.012 & 203 & 20 \\
\hline \multicolumn{7}{|l|}{ Negative words } \\
\hline Postcentral cortex & left & $(-44,-32,46)$ & 5.56 & 0.015 & 223 & 2 \\
\hline
\end{tabular}

\section{Imaging}

Cognitive Stroop. Task related BOLD responses during the BAL condition are presented in Table 2. IC words (contrast 1) were associated with an increased BOLD response in the left inferior parietal cortex (BA 40), left middle frontal cortex (BA 46), and right superior temporal cortex (BA 22). CC words (contrast 2) increased the BOLD response in the left inferior parietal (BA 40) and the right inferior frontal cortex (BA 45). IC minus CC words (contrast 3) was associated with an increased BOLD response in a left inferior frontal cluster (BA 44/45).

Since previous research (Compton et al., 2003; Bush et al., 1998) suggested that ACC activation is especially prone to the effects of practice, we conducted a post hoc analysis and investigated the BOLD response associated with IC minus $\mathrm{CC}$ words in the ACC (TD label bilateral anterior cingulate as ROI) in the first Stroop block the participants performed (for nine participants this was a BAL block, for six participants this was a TRP- block). The analysis showed an increased BOLD response in the ACC (BA 32) associated with Stroop interference. 

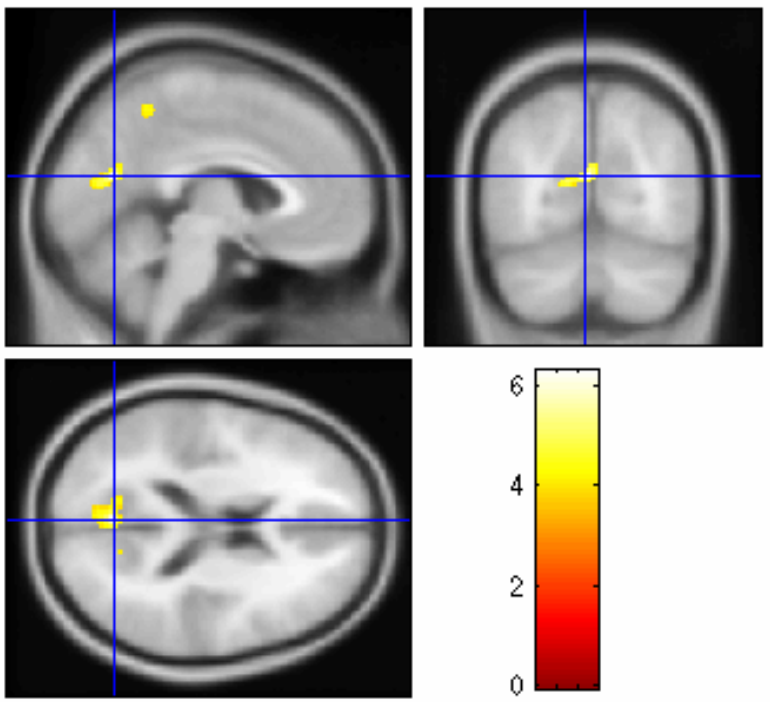

Figure 1. Whole brain analysis revealed that ATD increased BOLD response in the left calcarine gyrus during congruent colour words.
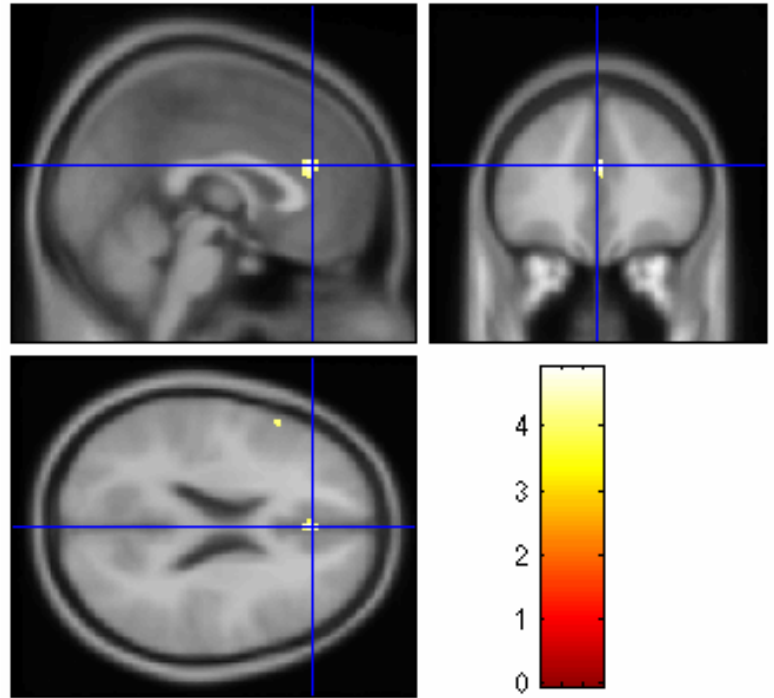

Figure 2. Region of interest analysis revealed that ATD increased the BOLD response in the anterior cingulate cortex when colour incongruent words were compared with colour congruent words in the first Stroop block the participants performed. 
The effects of ATD on the BOLD response during the cognitive part of the Stroop task are presented in Table 3. No effect of ATD was found during IC words (contrast 9). ATD increased the BOLD response in the left precuneus (BA 31) and cuneus (BA 18) during CC words (contrast 10) (see Figure 1). Whole brain analysis did not reveal ATD effects for contrast 11. To calculate the effect of ATD on IC minus CC words in the first Stroop block the participants performed, we first used a whole brain two samples (BAL or TRP-) t-test in which the TRPStroop blocks performed first were compared with BAL Stroop blocks firstly performed. Whole brain analysis did not reveal significant effects of ATD. Secondly, an ROI analysis was conducted: A $10 \mathrm{~mm}$ sphere was build around MNI $x=0, y=36, z=22$, which is the centre coordinate of the task-related BOLD response in the first Stroop block. This analysis showed that ATD increased the BOLD response in the ACC (BA 32) in the first Stroop block the participants performed (see Figure 2).

Table 3. Areas in which ATD increased the BOLD response during the cognitive Stroop task. $\mathrm{CC}=$ congruent colour words. IC = incongruent colour words.

\begin{tabular}{|c|c|c|c|c|c|}
\hline & $\begin{array}{c}\text { MNI } \\
\text { coordinates }\end{array}$ & T-value & $\begin{array}{c}\mathrm{P} \\
\text { corrected } \\
\text { cluster }\end{array}$ & $\begin{array}{c}\text { Number of } \\
\text { voxels }\end{array}$ & $\begin{array}{l}\text { Brodmann } \\
\text { area }\end{array}$ \\
\hline \multicolumn{6}{|c|}{ IC minus CC words 1 st Stroop block (ROI analysis) } \\
\hline $\begin{array}{l}\text { Anterior cingulate } \\
\text { cortex }\end{array}$ & $(0,36,22)$ & 4.37 & 0.023 & 9 & 32 \\
\hline \multicolumn{6}{|c|}{ CC words (whole brain analysis) } \\
\hline Left precuneus & $(-4,-68,18)$ & 6.28 & 0.004 & 256 & 31 \\
\hline Left cuneus & $(-10,-74,14)$ & 6.12 & & & 18 \\
\hline
\end{tabular}

Emotional Stroop. Positive words (contrast 5) were associated with an increased BOLD response in the left inferior temporal cortex (BA 20) and negative words (contrast 6) with an increased BOLD response in the left postcentral cortex (BA 2) (see Table 1). No significantly increased BOLD response was found for neutral words (contrast 4), positive minus neutral words (contrast 7) and negative minus neutral words (contrast 8). No effect of ATD was found on the BOLD response during emotional words (contrast 12 until 16) and overall activation (contrast 17). 


\section{Subjective Measures}

On the POMS questionnaire no effect of ATD was found on the subscales for depression $(\mathrm{F}(1,13)=0.6 ; \mathrm{P}=0.5)$, anger $(\mathrm{F}(1,13)=1.3 ; \mathrm{P}=0.3)$, fatigue $(\mathrm{F}(1,13)=4.0$; $\mathrm{P}=0.1)$ and tension $(\mathrm{F}(1,13)=1.0 ; \mathrm{P}=0.3)$. A significant main effect of Time $(\mathrm{F}(1,13)=6.4 ; \mathrm{P}<0.05)$ and a significant interaction effects of Time*Group $(\mathrm{F}(1,13)=6.4 ; \mathrm{P}<0.05)$ and Treat*Time $(\mathrm{F}(1,13)=5.6 ; \mathrm{P}<0.05)$ were found for vigour: participants whose second session was the TRP- session felt less vigorous after ATD. No effect of ATD on physical complaints was found $(F(1,13)=0.1$; $\mathrm{P}=0.7)$.

\section{Discussion}

The present study investigated the effect of ATD on performance and the BOLD response during a combined cognitive and emotional Stroop task. In the cognitive part of the Stroop task, we showed first that ATD increased the BOLD signal in the ACC when IC words were compared with CC words in the first block of the Stroop task. Secondly, ATD increased the BOLD response in the left precuneus (BA 31) and cuneus (BA 18) during CC words. At the behavioural level, ATD decreased the interference score for IC words. In the emotional part of the Stroop task, we showed that ATD increased the number of errors on negative words, but did not change the BOLD response.

\section{Cognitive Stroop}

Largely in accordance with the results of a meta-analysis by Laird et al. (2005), we found an increased BOLD response in the left inferior parietal, middle frontal, and the right superior temporal cortex related to IC words in the BAL condition. Interestingly, the current study did not find an increased BOLD response in the ACC during Stroop interference. A study carried out by Compton et al. (2003) also failed to find increased ACC activation during IC words. They suggested that this might be explained by practice effects and mention a study in their laboratory that showed increased ACC activation in the first, no ACC activation in the second, and decreased ACC activation in the last third of a Stroop interference condition. Furthermore, Bush et al. (1998) showed that the relative difference in the ACC activity between the interference and the neutral conditions decreased as subjects learned the task. In the present study the participants performed two blocks of the Stroop task during the practice session, (80 IC trials), two in the BAL (80 IC trials) and two in the TRP- session (80 IC trials). A post-hoc analysis revealed that the ACC was activated when IC words were compared with CC words in the first Stroop block the participants performed. This result confirms the hypothesis that practice decreased the BOLD response in the ACC. 
Post hoc ROI analysis revealed that ATD increased the BOLD response in the ACC (BA 32) when IC words were compared with CC words in the first Stroop block the participants performed. Since the ACC has been related to conflict monitoring and signalling the need for more cognitive control (Botvinick et al., 1999, 2004; Ridderinkhof et al., 2004 b; Kerns et al., 2004; Matsumoto and Tanaka, 2004), we hypothesize that the increased ACC activation triggered more cognitive control and thereby improved performance during Stroop interference. This hypothesis needs to be tested by future research. In line with these findings, Horacek et al. (2005) reported an increased BOLD response in the ACC (BA 23) after ATD during the interference condition of a Stroop task.

The finding of an increased response in the $\mathrm{dmPFC}$ is in line with a previous study (Evers et al., 2005) in which we showed that ATD increased the BOLD response in the dmPFC to negative feedback preceding a switch in response strategy. It should be noted, however, that the centre of the area with increased activation was located more ventrally in the present study ([MNI: $x=0, y=36$, $\mathrm{z}=22$ ] vs. [MNI: $\mathrm{x}=9, \mathrm{y}=39, \mathrm{z}=48]$ ). Based on a review study by Ridderinkhof et al. (2004 b), which showed that activation related to conflict is not only found in the ACC (BA 24 and 32) but also more dorsally (BA 6 and 8), we would like to speculate that the increased dmPFC response that was found in both studies possibly reflects the same underlying cognitive process. The response to IC trials as well as the response to negative feedback preceding a switch in response strategy might be related to conflict monitoring. In contrast, our research group found that ATD decreased the response in the dmPFC (BA 8; MNI: $x=0, y=46$, $\mathrm{z}=38$ ) after a response error followed by negative feedback in a Go/NoGo task in healthy male volunteers (unpublished data). Talbot and Cooper (2006) showed that ATD reduced the activation in the dorsal ACC (BA 32; MNI: $x=-6, y=38, z=24$ ) in healthy participants at rest. The present data confirm the suggestion of these authors that the dmPFC might be related to the effect of ATD on cognition in healthy participants. Obviously, more research is needed to examine the seemingly complex association between reduced 5-HT, the dmPFC and cognition.

ATD increased the BOLD response in the left precuneus (BA 31) and cuneus (BA 18) during CC trials. Previous ATD studies (Booij et al., 2005; Gallagher et al., 2003; Horacek et al., 2005; Schmitt et al., 2000; Sobczak et al., 2003) did not examine CC words since these studies used the three card version of the Stroop task. The present study used a modified Stroop task designed for event-related fMRI in which both IC and CC words were presented. We were therefore able to study the interference (slowing) and the facilitation (speeding) effect of the colour name. ATD increased the BOLD response in the cuneus (BA 18; part of area V2) and the precuneus (BA 31; part of area $\mathrm{V} 4$ ) during CC words. These areas are part 
of the parvocellular path, which is important in colour perception (for review see Gegenfurtner and Kiper, 2003). The present study suggests that ATD increases the BOLD response in brain areas related to colour perception during the reading of $\mathrm{CC}$ words. It is noteworthy that ATD did not affect the performance on CC words.

\section{Emotional Stroop}

In accordance with our hypothesis, we showed that participants reacted slower to negative than to neutral or positive words. Despite these differences in RTs, we did not find differences in BOLD response between these emotionally salient words. Compton et al. (2003) reported that multiple pilot studies in their laboratory indicated that emotion related brain activation only showed in a blocked design. It is likely that the presentation of the emotional words in the present study was too short to change the emotional state of the participants. Participants might have been distracted by these words, as shown in the RT data, without showing brain activity related to this emotional state.

Booij et al. (2005) showed that ATD increased interference levels for positive words on an emotional Stroop task. This increase was found in the high dose ATD condition $(100 \mathrm{~g})$, but not in the low-dose ATD condition $(50 \mathrm{~g})$. The present study used a $75 \mathrm{~g}$ AA mixture, which can be seen as a medium dose. This might explain why the increase in interference levels for positive words was present in our study, but did not reach significance $(\mathrm{F}(1,13)=2.1 ; \mathrm{p}=0.17)$. Instead, the present study showed that ATD increased the number of errors on negative words. Unfortunately, Booij et al. (2005) did not report the number and kind of errors made during the emotional Stroop task. Studies that investigated the processing of emotional words in depressed patients (e.g. Lim and Kim, 2005; Rinck and Becker, 2005) are in line with our results. These patients often have an emotional processing bias in favour of negative words that are related to their relevant concerns (e.g. Nunn et al., 1997).

A limitation of the present study is that we used female volunteers only. Since previous studies showed that ATD affects females and males differently (Booij et al., 2002; Harmer et al., 2003), the results of the present study are representative for a female population only.

To conclude, the present study confirms the suggested role for 5-HT in response conflict. It showed that ATD improved Stroop performance and increased the BOLD response in the ACC in the first Stroop block. However, more research is needed to examine the association between low 5-HT, the dmPFC and cognition. 


\title{
Chapter 5
}

\section{A genetic predisposition to depression interacts with the effect of acute tryptophan depletion during a Stroop task}

\author{
EAT Evers, FM van der Veen, J Jolles, NEP Deutz, JAJ Schmitt
}

\begin{abstract}
Background. The present study investigated the interaction between a genetic predisposition to depression and the effect of acute tryptophan depletion (ATD), a well-known method to temporarily reduce central serotonin (5-HT), on mood, cognition and brain activation. Methods. Healthy females with $(\mathrm{FH}+; \mathrm{n}=12)$ and without $(\mathrm{FH}-; \mathrm{n}=15)$ a positive family history of unipolar depression were tested in an ATD and a placebo session during a combined cognitive and emotional Stroop task in an event-related fMRI study. Results. ATD triggered a mood response in the $\mathrm{FH}+$ group. On the behavioral level, ATD decreased the interference score for incongruent (IC) versus congruent color (CC) words in the FH- group, decreased accuracy with regard to IC, $\mathrm{CC}$ and negative words in the $\mathrm{FH}$ - group and increased accuracy in relation to $\mathrm{IC}, \mathrm{CC}$ and negative words in the FH+ group. Individual mood changes did not correlate with performance changes. Activation in the anterior cingulate cortex (ACC) correlated with mood changes during Stroop interference: the more depressed after ATD, the more activation in the ACC. Conclusions. The present study showed that a genetic predisposition to depression influences the effect of a transient reduction of central 5-HT on mood and performance during a Stroop task.
\end{abstract}

\section{Acknowledgements}

We thank Olga Reneerkens, Frouke Nijhuis and Gabry Mies for test and analysis assistance and Jeroen van Deursen for radiographic assistance. This work was supported by a TOP grant (No. 912-02-050) from ZonMW-NWO and a grant from the Dutch Brain Foundation (Hersenstichting Nederland, 11F03(2).41). 


\section{Introduction}

Major depression, a disorder characterized by depressed mood and cognitive dysfunction, has been associated with disturbed serotonergic functioning (Risch and Nemeroff, 1992). To gain more insight into the etiology and symptoms of depression, acute tryptophan depletion (ATD) has been used as a model for depression. ATD is a well-established method to temporarily lower central serotonin (5-HT) (Nishizawa et al., 1997; Williams et al., 1999; Young et al., 1999). In line with cognitive disturbances found in depressed patients, ATD impaired memory consolidation (Riedel et al., 1999; Schmitt et al., 2000) and cognitive flexibility (Park et al., 1994; Rogers et al., 1999a) in healthy volunteers. In addition, ATD has been used to study individual vulnerability of the 5-HT system. Previous studies showed that risk factors for developing depression (e.g. the s/s genotype for the 5-HT transporter gene, previous depressive episodes, being female, a positive family history of affective disorder) trigger a mood response after ATD (Benkelfat et al., 1994; Booij et al., 2002; Ellenbogen et al., 1996; Klaassen et al., 1999; Neumeister et al., 2002; Riedel et al., 2002, 2003). Participants without a genetic susceptibility to depression do not show a mood response after ATD (for review see van der Does 2001).

Only few studies investigated the association between ATD, mood and cognitive performance in healthy volunteers with a genetic susceptibility to depression. Klaassen et al. (2002) showed that ATD impaired delayed recall of neutral and positive words, but not of negative words, during an affective verbal learning task in healthy volunteers with and without a family history of major depression. ATD did not affect mood in this study. Booij et al. (2005) showed that ATD induced a mood response and impaired the processing of positive words on an emotional Stroop task in remitted depressed patients. Munafo et al. (2006) showed that ATD induced a mood response and increased interference from social threat words in recovered depressed patients during an emotional Stroop task. It remains unclear whether ATD affects cognition via individual mood changes or independently affects mood and cognition. To gain more insight into the association between a transient lowering of central 5-HT, mood and cognition the present study examined the effect of ATD in healthy females with a family history of unipolar depression (FH+) during a combined cognitive and emotional Stroop task in an event-related fMRI paradigm. These data were directly compared with data from healthy females without such family history (FH-). The data of the FHgroup were reported by Evers et al. (in press b).

Previous ATD studies showed inconsistent effects of ATD on Stroop performance in healthy volunteers. Some studies showed that ATD improved (Coull et al., 1995; Evers et al., in press b; Rowley et al., 1997; Rosse et al., 1992; 
Schmitt et al., 2000), whereas other studies showed that ATD did not change Stroop performance (Gallagher et al., 2003; Horacek et al., 2005; Sobczak et al., 2002). Not only behavioral studies but also neuroimaging studies are inconclusive. In an event-related fMRI study Horacek et al. (2005) showed that ATD did not affect mood and performance, but did increase activation in the bilateral mediofrontal cortex, anterior cingulate cortex (ACC) and the dorsolateral prefrontal cortex during Stroop interference. In an earlier study (Evers et al., in press b), we showed that ATD did not affect mood, improved performance and increased the activation in the ACC during Stroop interference in the first Stroop block in healthy $\mathrm{FH}$ - females. In addition, we showed that ATD increased the activation in the left precuneus and cuneus during congruent color words. Factors like a genetic susceptibility to depression may influence not only the effect of ATD on mood, but also the effect of ATD on cognition and brain activation. Talbot and Cooper (in press) showed that increased individual sadness after ATD was associated with increased activation in the left subgenual ACC and the right inferior and middle temporal cortex in healthy participants.

The present study tested the following hypotheses: 1) ATD triggers a mood response in FH+ but not in FH- participants, 2) ATD affects performance and brain activation differently in $\mathrm{FH}+$ and $\mathrm{FH}$ - participants, 3) the mood response triggered by ATD is associated with the effect of ATD on Stroop performance and brain activation.

\section{Methods and materials}

Fifteen healthy females without a family history of affective disorder (FH-) performed a combined cognitive and emotional Stroop task in a balanced (BAL) and a tryptophan depleted (TRP-) session. This part of the data is presented in Evers et al. (in press b). The present study tested an additional group of healthy females with a family history of major depression $(\mathrm{FH}+)$ using the exact same paradigm, and directly compared the results of the FH- and the FH+ group. Mean ages and educational levels in the $\mathrm{FH}-$ and $\mathrm{FH}+$ groups are comparable (Van der Veen et al., submitted). For details about the FH- participants, experimental design, Stroop task, amino acid (AA) mixture, biochemical analysis, mood and health complaints questionnaires, image acquisition and pre-processing, see Evers et al. (in press b).

\section{Participants}

The present study included fourteen healthy female volunteers (between 19 and 42 years old; mean age 23.5; SE of mean 1.5) with a first-degree relative (father, mother, brother or sister) who had been diagnosed with unipolar depression. The 
family members gave permission to check the diagnosis with their general practitioners. The diagnosis of unipolar depression was confirmed for all relatives. The health states of the participants were checked by a medical questionnaire, which was evaluated by a medical doctor. All participants were free of present mental or physical illness, had never been seriously ill, did not use medication, had never used antidepressants or ecstasy, and were screened for MRI contraindications. This study was approved by the Medical Ethics Committee of Maastricht University Hospital and all participants gave written informed consent before inclusion. The participants were recruited by local advertisements and were paid 75 euros.

\section{Experimental design}

Participants were tested in a double-blind placebo-controlled within-subject design. When the participants arrived at the laboratory they first completed mood (the Profile of Mood States (POMS), McNair et al., 1988) and physical complaints questionnaires $(\mathrm{t} 0)$, then a blood sample was taken $(\mathrm{t} 0)$ and thereafter on AA drink (75 g) was consumed. After a four and a half hour break they filled out another set of questionnaires (t5) and a second blood sample was taken (t5). Thereafter the participants were scanned at the Maastricht University Hospital radiology department while they performed two blocks of the Stroop task (9 min each). Halfway through the scanning session a structural scan was made (10 min). In addition, the participants performed a facial recognition task (data will be presented elsewhere).

\section{Stroop task}

In this task every two seconds a word printed in colored ink (red, blue, green, yellow) was presented against a black background. The participants had to report the color of the ink in which the words were printed by pressing a button of a response box. In each test session the participants performed two Stroop blocks. Each Stroop block contained 40 congruent color (CC) words (e.g. 'red' written in red ink), 40 incongruent color (IC) words (e.g. 'red' written in blue ink), 24 positive, 24 negative and 24 neutral words in a semi-randomized order (never the same word type or color three times in a row). During CC and IC words the name of the color was the distracter, during emotional words the emotional meaning of the word was the distracter. Each block started with ten neutral words which were not included in the analysis. Before the first test session the participants were trained in a dummy scanner. In this practice session they performed two Stroop blocks that each contained $40 \mathrm{CC}$, 40 IC and 72 neutral words. The following performance measures were collected: the mean reaction time (RT) and the number of errors for IC, CC, positive, negative and neutral words, the interference score for IC versus CC words ((RT for IC words - RT for CC words)/ RT for CC 
words), the interference score for positive versus neutral words ((RT for positive words - RT for neutral words)/ RT for neutral words) and the interference score for negative versus neutral words ((RT for negative words - RT for neutral words)/ RT for neutral words).

\section{Data analysis}

Performance. The effect of ATD on the mean RTs and the number of errors was analyzed (SPSS version 11.5 for Windows) using a general linear model (GLM) repeated measurements design with Treatment (BAL or TRP-) and Word Type (IC and CC words, or neutral, positive and negative words) as within-subject variables, Group as between-subject variable and Order as a covariate. The effect of ATD on interference scores was analyzed using a GLM repeated measurements design with Treatment as a within-subject variable, Group as a between-subject variable and Order as a covariate.

Biochemical. The effect of ATD on plasma TRP levels and the TRP/ $/$ LNAA (LNAA: large neutral amino acids) ratios was analyzed using a GLM repeated measurements design with Time (t0 and $\mathrm{t5}$ ) and Treatment as within-subject variables, Group as a between-subject variable and Order as a covariate.

Subjective measurements. The effect of ATD was assessed for the summed score of the five mood dimensions of the POMS questionnaire and for the adverse effects using a GLM repeated measurements design with Time and Treatment as within-subject variables, Group as a between-subject variable and Order as a covariate.

Imaging. A simple hemodynamic response was used as a covariate in a GLM and a parametric estimate was generated for each voxel for IC, CC, neutral, positive and negative words (correct and incorrect). Individual contrast images were taken to a second level analysis, in which t-values were calculated for each voxel treating inter-subject variability as a random effect. The hemodynamic response function was modeled to the onset of the response. Firstly, we calculated task effects and the effect of ATD for the whole group (FH- and FH+, both BAL and TRP- data) using a one sample t-test. Thereafter, two samples t-tests were used to calculate i) the effect of Group on task effects and on the ATD effect and ii) the effect of Order on the ATD effect. The following contrasts were calculated to assess task effects: 1) IC minus CC words, 2) positive minus neutral words, 3) negative minus neutral words. The following contrasts were calculated to assess the effect of ATD: 4) IC minus CC words in the TRP- compared with IC minus CC words in the BAL condition ([IC - CC] x ATD), 5) [pos - neu] x ATD, 6) [neg - neu] x ATD, 7) all events (IC, CC, neutral, positive and negative words) $\mathrm{x}$ ATD. Based on a meta-analysis of the manual Stroop task (Laird et al., 2005), five regions of interest (ROIs) were defined $(10 \mathrm{~mm}$ spheres around centre coordinates; SPM2 small volumes correction) for contrast 1: the left inferior junction (Talairach 
coordinates (TAL): $x=-43, y=4, z=35$ ), the ACC (TAL: $x=3, y=16, z=41$ ), the left inferior parietal cortex (TAL: $x=-47, y=-40, z=47$ ), the left middle frontal gyrus (TAL: $\mathrm{x}=-34, \mathrm{y}=21, \mathrm{z}=24$ ) and the left precuneus (TAL: $\mathrm{x}=-21, \mathrm{y}=-70, \mathrm{z}=37$ ). ROIs for contrast 4 till 6 were built around the centre coordinates of the task related activation (10 mm spheres; SPM2 small volumes correction). For whole brain and ROI analyses, results are reported when Pcluster-corrected $<0.05$.

Since previous research (Bush et al., 1998; Compton et al., 2003; Evers et al., in press b) suggested that ACC activation is especially prone to the effects of practice, we investigated brain activation related to IC minus CC words in the first Stroop block. The following analyses were used: a one sample t-test to examine the activation for the whole group (FH- and FH+, both BAL and TRP- data) and two samples t-tests to examine to effect of Group and the effect of ATD. We used the bilateral ACC as a ROI (WFU PickAtlas, TD label bilateral ACC).

In this paper coordinates are reported as Montreal Neurological Institute (MNI) coordinates. Only to determine anatomical labels (The Talairach Daemon; http://ric.uthscsa.edu/TDapplet/), the coordinates of the significant areas were converted to Talairach coordinates (http://www.mrc_cbu.cam.ac.uk/imaging).

\section{Results}

In the $\mathrm{FH}+$ group fourteen participants were included. However, imaging data from one participant was missing because of technical problems and another participant was excluded due to bad performance: she made 88 errors in the TRPcondition which was more than 3 standard deviations from the average (mean 4.3; $\mathrm{SD}=3.7$ ). Data is presented for twelve $\mathrm{FH}+$ (four started in the BAL and eight in the TRP-condition) and for fifteen FH- participants (nine started in the BAL and six in the TRP- condition).

\section{Biochemical results}

Statistical analysis showed a main effect of Treatment $(F(1,11)=22.9 ; \mathrm{p}=0.001)$, a main effect of Time $(\mathrm{F}(1,11)=15.6 ; \mathrm{p}=0.002)$, an interaction between Time and Order $(\mathrm{F}(1,11)=7.9 ; \mathrm{p}=0.017)$, Treatment and Time $(\mathrm{F}(1,11)=32.9 ; \mathrm{p}=0.000)$, and Treatment, Time and Order $(\mathrm{F}(1,12)=7.9 ; \mathrm{p}=0.017)$ for plasma TRP levels. Post hoc analyses showed that the BAL mixture increased plasma TRP, when participants started in the TRP-condition. In addition, post hoc analyses showed that the TRP- mixture reduced plasma TRP while the BAL mixture increased plasma TRP. No effect of Group was found. Statistical analysis showed a main effect of Treatment $(\mathrm{F}(1,9)=5.3 ; \mathrm{p}=0.046)$ and an interaction between Treatment and Time $(\mathrm{F}(1,9)=12.6 ; \mathrm{p}=0.005)$ for the TRP/LLNAA ratio. A post hoc pairedsample t-test showed that the ratio decreased after the TRP- mixture $(t=13.8$; 
$\mathrm{p}=0.000$; ratio is $0.12 / 0.01$ at $\mathrm{t} 0$ and $0.01 / 0.00$ at $\mathrm{t} 5)$ and was unchanged after the BAL mixture ( $\mathrm{t}=-0.3 ; \mathrm{p}=0.8$; ratio is $0.11 / 0.00$ at $\mathrm{t} 0$ and $0.11 / 0.00$ at $\mathrm{t} 5)$. No effect of Group was found.

\section{Subjective Measures}

Statistical analysis showed an interaction between Treatment and Time $(\mathrm{F}(1,24)=5.6 ; \mathrm{p}=0.027)$, Treatment, Time and Order $(\mathrm{F}(1,24)=5.0 ; \mathrm{p}=0.035)$, and Treatment, Time and Group $(\mathrm{F}(1,24)=5.0 ; \mathrm{p}=0.012)$ for the summed POMS score. Post hoc paired-sample t-tests showed that ATD increased the summed score in the $\mathrm{FH}+$ group $(\mathrm{t}=-2.3 ; \mathrm{p}=0.04)$. Because of the specific hypothesis that ATD increases the depression score in FH+ participants, we tested the effect of ATD on the depression score for both groups separately using a paired-sample t-test (BAL t5 vs. TRP- t5). ATD tended to increase the depression score in the FH+ group $(\mathrm{t}=2.1 ; \mathrm{p}=0.059)$, but not in the $\mathrm{FH}$ - group $(\mathrm{t}=-0.11 ; \mathrm{p}=0.9)$. Because of our a priori hypothesis that ATD triggers a mood response in $\mathrm{FH}+$ but not in $\mathrm{FH}-$ participants, we tested this hypothesis one-sidedly. We therefore concluded that ATD significantly increased $(\mathrm{F}(1,13)=4.5 ; \mathrm{p}=0.03)$ the depression score in the $\mathrm{FH}+$ group. ATD did not influence adverse effects.

\section{Performance}

\section{Cognitive Stroop.}

RT, accuracy and the interference score are presented in Table 1. Statistical analysis showed an interaction between Treatment and Group $(\mathrm{F}(1,24)=6.2$; $\mathrm{p}=0.02$ ) for the number of errors. The number of errors increased in the FH- but decreased in the $\mathrm{FH}+$ group. Statistical analysis revealed an effect of Word Type $(\mathrm{F}(1,24)=8.0 ; \mathrm{p}=0.009)$ for RTs. Participants were slower on IC than on CC words. No other effects on RTs were found. Statistical analysis showed an effect of Treatment $(\mathrm{F}(1,24)=4.6 ; \mathrm{p}=0.041)$ for the interference score. In addition, a marginal significant interaction between Treatment and Order $(F(1,24)=4.0$; $\mathrm{p}=0.056)$ and Treatment and Group $(\mathrm{F}(1,24)=4.2 ; \mathrm{p}=0.053)$ was found. Post hoc paired-sample t-tests that examined the latter interaction showed that ATD decreased the interference score in the $\mathrm{FH}-$ group $(\mathrm{t}=-2.3 ; \mathrm{p}=0.035)$. To test the hypothesis that mood changes after ATD influence performance, a simple regression analysis was performed. We calculated a 'delta depression' score (TRP[t5 - t0] - BAL [t5 - t0]) and correlated this score with the 'delta interference' score (TRP- minus BAL) and the 'delta number of errors' score (TRP- [CC + IC] minus BAL $[\mathrm{CC}+\mathrm{IC}])$. The mean 'delta depression' score was $-16.3(\mathrm{SE}=16.9)$ for the FH- group, and 29.0 ( $\mathrm{SE}=26.7$ ) for the $\mathrm{FH}+$ group. Talbot and Cooper (in press) showed that in a male FH- group individual mood changes after ATD were correlated with changes in brain activation. Therefore we correlated not only the mood changes of the FH+ group with performance and brain activation, but used 
the whole group $(n=27)$ to assess the influence of mood changes on performance and brain activation. No correlation was found between the 'delta depression' and the 'delta interference' score $(\mathrm{r}=0.05 ; \mathrm{p}=0.81)$, and no correlation was found between the 'delta depression' and the 'delta number of errors' score $(r=0.07$; $\mathrm{p}=0.82$ ).

\section{Emotional Stroop.}

RT, accuracy and the interference scores are presented in Table 1. Statistical analysis showed an interaction between Treatment, Word Type and Group $(\mathrm{F}(1,24)=4.9 ; \mathrm{p}=0.017)$. Post hoc repeated measured analyses per word type showed an interaction between Group and Treatment $(\mathrm{F}(1,24)=9.7 ; \mathrm{p}=0.005)$ for negative words. In the $\mathrm{FH}$ - group the number of errors on negative words increased after $\mathrm{ATD}$, in the $\mathrm{FH}+$ groups the number of errors on negative words decreased after ATD. No effects were found for RT and interference scores. To test the hypothesis that mood changes influence performance, a simple regression analysis was performed. No correlation was found between the 'delta depression' and the 'delta number of errors on negative words' score (TRP- minus BAL) (r=$0.32 ; \mathrm{p}=0.14)$.

Table 1. Performance data. Mean reaction times (ms), the percentage incorrect responses and the interference scores, with errors of the mean

\begin{tabular}{lcccc}
\hline & \multicolumn{2}{c}{ FH- group $(\mathrm{n}=15)$} & \multicolumn{2}{c}{ FH+ group $(\mathrm{n}=12)$} \\
Event type & BAL condition & TRP- condition & BAL condition & TRP- condition \\
\hline $\begin{array}{l}\text { Mean reaction time } \\
\text { incon }\end{array}$ & $768(24)$ & $736(20)$ & $748(33)$ & $735(28)$ \\
con & $650(16)$ & $636(16)$ & $634(20)$ & $608(21)$ \\
neu & $705(29)$ & $694(25)$ & $666(22)$ & $640(21)$ \\
pos & $687(26)$ & $698(26)$ & $662(23)$ & $643(21)$ \\
neg & $717(28)$ & $705(30)$ & $671(27)$ & $656(24)$ \\
Interference $(\%)$ & & & & \\
incon versus con & $19(2)$ & $13(2)$ & $18(3.7)$ & $21(4.0)$ \\
pos versus neu & $-2.3(1)$ & $0.5(1)$ & $-0.05(1.2)$ & $0.05(1.1)$ \\
neg versus neu & $1.9(1)$ & $1.4(1)$ & $0.6(1.2)$ & $0.2(1.0)$ \\
Percentage errors & & & & \\
incon & $7.5(0.6)$ & $9.0(0.8)$ & $6.7(0.8)$ & $5.0(0.9)$ \\
con & $3.3(0.4)$ & $4.2(0.5)$ & $1.9(0.2)$ & $0.9(0.2)$ \\
neu & $4.8(0.4)$ & $3.0(0.3)$ & $3.0(0.4)$ & $1.4(0.1)$ \\
pos & $4.8(0.3)$ & $4.8(0.4)$ & $2.8(0.3)$ & $2.4(0.2)$ \\
neg & $3.8(0.3)$ & $8.3(0.4)$ & $4.9(0.2)$ & $3.1(0.2)$ \\
\hline
\end{tabular}




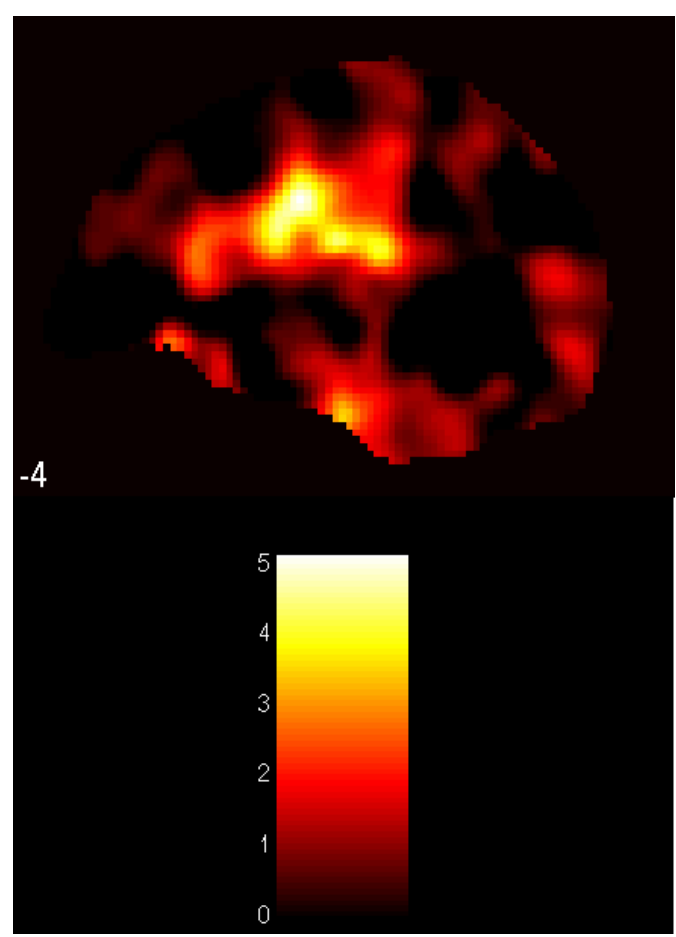

Figure 1. This figures shows the response in the anterior cingulate gyrus that correlated with the delta depression score for the contrast [incon con] x TRP.

\section{Imaging}

Cognitive Stroop.

Task related activation revealed by whole brain and ROI analyses is presented in Table 2. IC compared with CC words (contrast 1) were associated with increased activation in the left superior parietal cortex (BA 7; whole brain analysis), the right medial frontal gyrus (BA 8; ROI analysis) and left middle frontal gyrus (BA 9/46; ROI analysis). The contrast that compared IC with CC words in the first Stroop block was associated with increased activation in the ACC (BA 32; ROI analysis). No effect of ATD was found on brain activation related to IC minus CC words (contrast 4 and IC minus CC in first Stroop block). No effects of Group and Order were found for task-related activation and for the effect of ATD. In our earlier report about the FH- data (Evers et al., in press b) we showed that ATD increased the response in the ACC during the first Stroop block 
the FH- participants performed. A post hoc two sample t-test (FH+ data only) showed that ATD did not change the ACC response in the FH+ group during Stroop interference in the first Stroop block. A positive correlation between the 'delta depression' score and the contrast [IC minus CC] x ATD was found for a large ACC cluster (Pcluster- corrected $=0.000 ; 763$ voxels) which contained two ACC sub clusters (BA 24, MNI [-4, -6, 34], T=5.11; BA 24, MNI [16, 2, 32], T=4.95). See Figure 1.

\section{Emotional Stroop.}

Whole brain analysis showed more activation during positive than during neutral words in the cerebellum (contrast 2). No brain regions that were more activated during negative than during neutral words (contrast 3). No effect of ATD was found on brain activation related to positive minus neutral (contrast 5), negative minus neutral words (contrast 6), and overall brain activation (contrast 7). No effect of Group and Order was found for task-related activation and the effect of ATD. No regions were found that correlated with the 'delta depression' score.

Table 2. Task related activation revealed by whole brain and ROI analyses.

\begin{tabular}{|c|c|c|c|c|c|c|}
\hline & $\begin{array}{l}\text { Hemi- } \\
\text { sphere }\end{array}$ & MNI coordinates & $\begin{array}{c}\mathrm{T}- \\
\text { value }\end{array}$ & $\begin{array}{c}\text { Pcluster- } \\
\text { correct }\end{array}$ & $\begin{array}{c}\text { Number of } \\
\text { voxels }\end{array}$ & $\mathrm{BA}$ \\
\hline \multicolumn{7}{|l|}{ IC minus $C C$ words } \\
\hline \multirow{2}{*}{$\begin{array}{l}\text { Superior par. } \\
\text { cortex }\end{array}$} & \multirow[t]{2}{*}{ Left } & $(-34,-54,50)$ & 4.35 & \multirow[t]{2}{*}{0.046} & \multirow[t]{2}{*}{188} & \multirow[t]{2}{*}{7} \\
\hline & & $(-30,-64,48)$ & 4.28 & & & \\
\hline Medial fr. gyrus* & Right & $(6,20,40)$ & 3.99 & 0.019 & 18 & 8 \\
\hline \multirow{2}{*}{ Middle fr. gyrus* } & \multirow{2}{*}{ Left } & $(-42,20,22)$ & 4.17 & \multirow{2}{*}{0.027} & \multirow[t]{2}{*}{10} & \multirow[t]{2}{*}{$9 / 46$} \\
\hline & & $(-44,20,28)$ & 4.13 & & & \\
\hline \multicolumn{7}{|c|}{ IC minus CC words during first Stroop block } \\
\hline $\mathrm{ACC}$ & Left & $(-2,32,18)$ & 4.14 & 0.045 & 32 & 32 \\
\hline \multicolumn{7}{|c|}{ Positive minus neutral words } \\
\hline \multirow[t]{2}{*}{ Cerebellum } & \multirow[t]{2}{*}{ Right } & $(38,-64,-12)$ & 5.82 & \multirow[t]{2}{*}{0.002} & \multirow[t]{2}{*}{232} & \\
\hline & & $(42,-70,-24)$ & 4.31 & & & \\
\hline \multicolumn{7}{|c|}{ Negative minus neutral words } \\
\hline \multicolumn{7}{|c|}{ No significant activation clusters were found } \\
\hline
\end{tabular}

* ROI analysis 


\section{Discussion}

The present study compared the effect of a transient lowering of central 5-HT on mood, performance and brain activation during a combined cognitive and emotional Stroop in females with a family history of unipolar depression $(\mathrm{FH}+)$ with the effect in females without such family history (FH-). The first hypothesis, that ATD triggers a mood response in the FH+ but not in the FH- group, was confirmed. The second hypothesis, that ATD affects performance differently in the $\mathrm{FH}+$ than in the FH- group, was confirmed for the interference score for IC compared with $\mathrm{CC}$ words (ATD decreased interference score in the $\mathrm{FH}$ - group only) and the number of errors on CC, IC and negative words (ATD decreased accuracy in the $\mathrm{FH}$ - and increased accuracy in $\mathrm{FH}+$ group). The hypothesis that the mood response triggered by ATD is associated with differences in performance between groups was not confirmed. However, we showed that the activation in the ACC (BA 24) correlated with the 'delta depression' score for the effect of ATD on Stroop interference ([incon - con] x ATD): the more depressed after ATD, the more activation in the ACC during Stroop interference.

\section{Performance}

The present study showed that ATD decreased the interference score in the FHgroup. This finding is in agreement with previous studies that showed that ATD improved performance during a Stroop interference task (Coull et al., 1995; Rosse et al., 1992; Rowley et al., 1997; Schmitt et al., 2000). We showed that the interference score decreased for the FH- group, but was unaffected for the $\mathrm{FH}+$ group. This effect does not seem to be mediated by a mood effect, since increased depressed mood was not associated with the effects of ATD on the interference score. This finding is in agreement with Wagner et al. (2006) who did not find a correlation between the interference score and the symptom severity in depressed patients during a Stroop task.

Furthermore, we showed an interaction between ATD and Group for the accuracy on CC, IC and negative words. Previous ATD studies did not report the number of errors during a Stroop task. The present study showed that accuracy is an informative measure for the assessment of ATD effects. The interaction between Group and accuracy for negative words is not in agreement with the mood congruency bias which states that attention is directed towards emotional words that fit the current mood state (e.g. Beck 1967). According to the mood congruency bias, FH- participants without a mood effect are not distracted more by negative than by positive or neutral words. FH+ participants who do show a mood effect are more distracted by the negative than by positive or neutral words and therefore perform worse on negative words. The opposite pattern was found in 
the present study. In addition, two other ATD studies reported results that are not in agreement with the mood congruency bias. Booij et al. (2005) showed that a high-dose of ATD induced a mood response and increased the interference for positive but not for negative words in remitted patients. Van der Veen et al. (2006) showed that ATD increased the number of positively rated words during the encoding phase of a visual verbal episodic memory task. This suggests that the mood congruency bias is not an adequate model to explain the effect of ATD on performance during cognitive tasks in which emotional words or ratings are involved.

The finding that ATD decreased accuracy in the FH- group but increased accuracy in the FH+ group is a new and intriguing. Schmitt et al. (2000) suggested that improved performance after ATD on a Stroop and a dichotic listening task demonstrates improved focused attention, possibly linked to the removal of inhibitory activation by 5 -HT. The present study seems to suggest that attention might be more improved in $\mathrm{FH}+$ than in $\mathrm{FH}-$ volunteers. Obviously, this hypothesis needs to be tested by future research.

Contrary to our hypothesis, the present study showed that the effect of ATD on performance did not correlate with the effect of ATD on mood. These results suggest that the effect of ATD on mood and the effect of ATD on cognition are two independent effects. In other words, these results suggest that cognitive effects in this task are not triggered by mood changes, and the mood changes are not triggered by cognitive effects.

\section{Neuroimaging}

Largely in agreement with previous research (for review see Laird et al., 2005), we showed that Stroop interference (IC compared with CC words for both the $\mathrm{FH}+$ and the $\mathrm{FH}$ - group) was associated with activation in the left superior parietal cortex (BA 7), the right medial cortex (BA 8), and the left middle frontal gyrus (BA 9/46). The ACC (BA 32) was activated during Stroop interference in the first Stroop block the participants performed.

No effect of ATD and Group was found on brain activation associated with Stroop interference. In our earlier report about the FH- data (Evers et al., in press b), we showed that ATD decreased the interference score in the FH- group and increased BOLD response in the ACC (BA 32) when IC words were compared with CC words in the first Stroop block. We hypothesized that the increased ACC response might have triggered more cognitive control and thereby increased performance on the Stroop task. The findings of the present study are consistent with this suggestion: ATD did not increase the ACC activation and did not change the interference score in the $\mathrm{FH}+$ group. 
Interestingly, the BOLD response in the ACC (BA 24) correlated with the 'delta depression' score for the [IC - CC] x ATD contrast: ATD induced depressed mood that was related to increased activation in the ACC during Stroop interference. The ACC has been associated with mood change after ATD in two previous PET studies. In line with the present study, Talbot and Cooper (in press) showed that increased depressed mood was associated with increased activation in the subgenual ACC (BA 25) in healthy males at rest. However, the location of these responses within the ACC differed, and in the Talbot and Cooper study participants were at rest, while in the present study the participant performed a Stroop interference task. Smith et al. (1999) showed that increased depressed mood after ATD was associated with reduced activation in the ventral ACC (BA 24) in recovered depressed patients at rest and reduced the activation in the ACC (BA 32) in recovered depressed patients during a paced verbal fluency task. These studies suggest that in healthy participants depressed mood after ATD is associated with an increased response in the ACC, whereas in depressed patients an increased depressed mood after ATD is associated with a decreased response in the ACC. Interestingly, Wagner et al. (2006) reported that unmedicated depressed patients showed increased activation in the rostral ACC (BA 32) during Stroop interference. However, in this study the Hamilton depression score did not correlate with the ACC activation. These studies suggest that the ACC plays a role in the interaction between mood and cognition.

The present study confirms the suggestion of previous research that a blocked design is more appropriate than an event-related design for studying brain activation associated with emotional words (Compton et al., 2003). However, we showed that positive words were associated with increased activation in the cerebellum. Whereas it has long been thought that the cerebellum is involved in motor function only, recent studies suggest that the cerebellum is involved in emotion as well (Schmahmann and Caplan, 2006; Bermpohl et al., 2006). This suggestion is confirmed by the present study.

In conclusion, the present study showed that a genetic susceptibility to depression influences the effect of ATD on mood and performance during a combined cognitive and emotional Stroop task. Interestingly, the effect of ATD on performance did not correlate with the effect of ATD on mood. 
Chapter 5 


\title{
Chapter 6
}

\section{The effect of tryptophan depletion on the error-related negativity}

\author{
EAT Evers, FM van der Veen, NEP Deutz, EFPM Vuurman, J Jolles
}

\begin{abstract}
Rationale. The error-related negativity (ERN) is an event-related brain potential (ERP) component that is found around $100 \mathrm{~ms}$ after a response error and is thought to originate from the anterior cingulate cortex. In line with the suggestion that dopamine (DA) is involved in performance monitoring, previous studies showed that the ERN is affected by DA manipulations. However, the role of serotonin $(5-\mathrm{HT})$ during performance monitoring remains unclear. Some studies showed that 5-HT is involved in performance monitoring, while other studies did not show an effect of a 5-HT manipulation on the ERN. Objectives. The aim of the present study is to examine the effect of a transient lowering of 5-HT, using the method of acute tryptophan depletion (ATD), on the ERN. Methods. Twenty healthy male volunteers performed an Eriksen Flanker Task during a balanced and a tryptophan depletion session in an electroencephalogram (EEG) study. Results. On the behavioral level, ATD during the first session prevented faster responding on the second balanced session. With regard to the ERP data, ATD did not change the amplitude of the ERN. Conclusion. The present study suggests that a transient lowering of 5-HT does not change the ERN during an Eriksen Flanker task in healthy male volunteers.
\end{abstract}

\section{Acknowledgements}

We thank Floortje Smeets for test assistance. This work was supported by a TOP grant (No. 912-02-050) from ZonMW-NWO. 


\section{Introduction}

The error-related negativity (ERN) is an event-related brain potential (ERP) component that is found around $100 \mathrm{~ms}$ after a response error. The ERN is thought to originate from the anterior cingulate cortex (ACC) (Dehaene et al., 1994; Veen and Carter, 2002). Holroyd and Coles (2002) have proposed that the ERN reflects a reinforcement signal carried by the mesencephalic dopamine (DA) system to the ACC. According to this theory the ERN is related to outcomes of events that are worse than expected. In line with this theory, Zirnheld et al. (2004) and de Bruijn et al. (in press) showed that the DA antagonist haloperidol attenuated the ERN amplitude during an Eriksen Flanker Task. In accordance, another study by de Bruijn et al. (2004) showed that the ERN is enlarged by the indirect DA agonist amphetamine. These studies confirm the suggestion that DA is implicated in performance monitoring.

Two previous studies suggest that serotonin (5-HT) does not play a role in performance monitoring. De Bruijn et al. (2004; in press) showed that the ERN was unaffected by mirtazapine, a compound that affects histaminergic and 5-HT pathways, and paroxetine, a selective 5-HT reuptake inhibitor. In contrast, in a previous study our research group showed that a transient lowering of 5-HT attenuated the activation in the dorsomedial prefrontal cortex (dmPFC) after a response error followed by negative feedback in a Go/NoGo task (Evers et al., in press a). In addition, Fallgatter et al. (2004) reported that carriers of the short allele variant of a functional length variation in the transcriptional control region of the 5-HT transporter (5-HTT) gene showed a larger ERN compared to carriers of two long alleles.

To gain more insight into these contrasting effects of 5-HT manipulations on error processing, the present study investigated the effect of acute tryptophan depletion (ATD) on the ERN during an Eriksen Flanker Task (Eriksen and Eriksen, 1974) in healthy male volunteers. ATD is an established method to temporarily lower central 5-HT (Nishizawa et al., 1997; Williams et al., 1999; Young et al., 1999). The same Eriksen Flanker Task was used as in Bruijn et al. (2004). To the best of our knowledge, the present study is the first study to investigate the effect of ATD on the ERN. Based on the studies by de Bruijn et al. (2004; in press), that showed no effect of mirtazipine and paroxetine on the ERN, we expected that ATD would not affect the ERN during an Eriksen Flanker Task. 


\section{Materials and methods}

\section{Participants}

Twenty healthy males participated in this study (between 19 and 38 years old; mean age 23.2; SE of mean 1.0), which was approved by the Medical Ethics Committee of the Maastricht University Hospital. Participants were recruited by local advertisements. Their health was checked by a medical questionnaire, which was evaluated by a medical doctor. The participants did not suffer from present illnesses, had not been seriously ill in the past, did not use medication, had never been diagnosed with depression and had never used antidepressants or ecstasy. All participants gave written informed consent, and were paid 75 euros.

\section{Experimental design}

The participants were tested twice: in a balanced (BAL) and a tryptophan depletion (TRP-) session. These test sessions were at least one week apart. When the participants came to the laboratory they had fasted overnight and had used a low protein breakfast. When they arrived they first completed a mood and physical complaints questionnaire, where after a blood sample was taken before they consumed an amino acid (AA) drink. During the following four hour break they were only allowed to eat low protein food and drink decaffeinated beverages. After this break once again the questionnaires were completed, a blood sample was taken and the participants were then prepared for the test session. Participants were tested between 4.5 and 5.5 hours after they consumed the AA drink. The test session consisted of 6 short blocks ( 6 min each) of the Eriksen Flanker Task, and two longer blocks (10 min each) of a Time Estimation Task. The results of the Time Estimation Task will be presented elsewhere.

\section{Eriksen Flanker Task}

In the present study the task described by de Bruijn et al. (2004) was used. In their paper a detailed description of the task can be found. In this task five letters were presented centrally on a computer screen: HHHHH, HHSHH, SSSSS or SSHSS. When all five letters were the same the stimulus was labeled congruent (CO), otherwise incongruent (IC). The participants were instructed to respond with two alternative responses: a right hand response when the middle letter was an $\mathrm{H}$ and a left hand response when the middle letter was an S, as fast and accurate as possible. Before the actual testing (for both sessions) the participants performed a practice block that was used to determine the individual reaction time (RT) threshold (mean RT training session + 0.5 SD). This threshold was used to keep performance difficulty constant between participants and between sessions. Feedback was given after each response: a yellow rectangle after a correct 
response, a red rectangle after an incorrect response and a blue rectangle after a response that exceeded the individual RT threshold.

For both behavioral and ERP analyses all responses with RTs faster than 150 ms were removed (in total $1.0 \%$ of the responses made). Responses made within $150 \mathrm{~ms}$ after stimulus presentation were considered to be too fast for conscious processing. The following performance measures were used: (1) The percentage of errors on $\mathrm{CO}$ and IC trials (per trial type: [[total errors per session - total number non responses per session] / [300 - total number of responses faster than $150 \mathrm{~ms}$ ]] $\mathrm{x}$ 100). (2) The mean RTs on correct and incorrect CO and IC trials. Behavioral adjustments were measured by (3) post-error slowing, which is computed as the difference between the mean RT on a correct trial after a correct trial (post-correct) and the mean RT on a correct trial after in incorrect trial (post-error). Post-error slowing is based on the notion that the RT is increased on a trial after an error trial, reflecting a change in response strategy (Rabbitt 1966). (4) Another measure of behavioral adjustments is the sequential congruency effect, which is computed as the difference between the mean RT on an IC trial after an IC trial (IC-IC) and the mean RT on an IC trial after a CO trial (IC-CO). This measure is based on the notion that the RT on a trial is influenced by the previous trial type. Typically, an IC trial lead to a faster response on the next trial.

To analyze the different performance measures, a GLM repeated measurements design was used with Trial Type and Treatment (BAL or TRP-) as within-subject variables and Order (TRP- or BAL first) as a between-subject variable. The following Trial Types were compared:

1) percentage of errors on $\mathrm{CO}$ versus percentage of errors on $\mathrm{IC}$ trials

2a) mean RT on correct $\mathrm{CO}$ versus mean RT on correct IC trials

2b) mean $\mathrm{RT}$ on incorrect $\mathrm{CO}$ versus mean $\mathrm{RT}$ on incorrect IC trials

2c) mean RT on correct $\mathrm{CO}$ versus mean $\mathrm{RT}$ on incorrect $\mathrm{CO}$ trials

2d) mean RT on correct IC versus mean RT on incorrect IC trials

3) post-error versus post-correct

4) IC-IC versus IC-CO

\section{Questionnaires}

A visual analogue version of the Profile of Mood States (POMS) was used to assess mood (McNair et al., 1988). This questionnaire consists of 32 bipolar sets of adjectives, which measure five mood dimensions: anger, depression, fatigue, tension and vigor. Adverse effects, 31 items, were registered and scored on a 4 points scale from 'no complaint at all' (0) to 'severe complaint' (4). A total score was calculated by adding the scores on the individual items. The effect of ATD on mood and adverse effects was analyzed using a GLM repeated measurements 
design with Time (t0 and $\mathrm{t} 5$ ) and Treatment as within-subject variables and Order as a between-subject variable.

\section{Acute tryptophan depletion and biochemical analysis}

We used a $75 \mathrm{~g}$ AA mixture (for the exact amount and the determination of the AAs see Evers et al., in press a and b). Blood samples $(10 \mathrm{ml})$ were taken to determine the plasma tryptophan (TRP: the precursor of 5-HT) levels and the TRP/LLNAA ratios ([TRP] / [tyrosine + leucine + phenylalanine + isoleucine + valine]) for each participant. Plasma TRP levels and the ratios were analyzed using GLM repeated measurements with Time and Treatment as within-subject variables and Order as a between-subject variable. Paired-sample t-tests were used to compare baseline measurements.

\section{EEG acquisition}

EEG data was recorded in a controlled environment with a Neuroscan 4.3 / NuAmps EEG recording system (Neuroscan Inc, El Paso, TX, USA). Because of our special interest in the effect of ATD on the ERN we acquired signals from the following seven electrode positions: F3, FZ, F4, C3, CZ, C4 and PZ (in relation to A1-A2), which were positioned according to the international 10-20 system (Munday 2005). In addition, a vertical EOG signal was measured from an electrode placed below the right eye. The signals were sampled at a frequency of $500 \mathrm{~Hz}$, with A/D conversion of 22-bit with a frequency response of 0.01-40 Hz. All electrode impedances were below $8 \mathrm{k} \Omega$ before the start of the experiment.

\section{ERP analyses}

The EEG data was analyzed with NeuroScan 4.3 software. The EEG signal was divided into epochs from $200 \mathrm{~ms}$ before until $500 \mathrm{~ms}$ after the response (response locked). Preprocessing consisted of the following steps: ocular artifact correction (tool in Neuroscan 4.3), baseline correction, linear detrend and artifact rejection (EEG epochs with signal lower than $-50 \mu \mathrm{V}$ and higher than $50 \mu \mathrm{V}$ were removed). After pre-processing individual means per block were used to calculate weighted means per session per participant for correct and incorrect responses. These individual means were then used in the group analysis. To calculate the effect of ATD on the ERN the most positive value between $-80 \mathrm{~ms}$ and $80 \mathrm{~ms}$ was subtracted from the most negative value between $0 \mathrm{~ms}$ and $200 \mathrm{~ms}$ (de Bruijn et al., 2004), for each participant for each session. A GLM repeated measurements design was used with Treatment as a within-subject and Order as a betweensubject variable. 


\section{Results}

\section{Biochemical levels}

Data was complete for all twenty participants. Analyses showed a main effect of Time $(\mathrm{F}(1,18)=6.2 ; \mathrm{p}=0.02)$, an interaction between Treatment and Order $(\mathrm{F}(1,18)=59.1 ; \mathrm{p}=0.000)$ and between Treatment, Order and Time $(\mathrm{F}(1,18)=119.4$; $\mathrm{p}=0.000)$ for plasma TRP. ATD lowered the mean plasma TRP level by $70 \%$. Mean TRP level in the BAL condition was $50.8 \mu \mathrm{M}(\mathrm{SE}=2.7)$ at t0 and $101.5 \mu \mathrm{M}$ $(\mathrm{SE}=8.1)$ at $\mathrm{t} 5$. Mean TRP levels in the TRP- condition were $51.0 \mu \mathrm{M}(\mathrm{SE}=1.6)$ at t0 and $15.1 \mu \mathrm{M}(\mathrm{SE}=1.6)$ at $\mathrm{t} 5$. No significant difference in plasma TRP $(\mathrm{T}=-0.4$; $\mathrm{p}=0.7$ ) was present at baseline ( $\mathrm{t} 0$ ) between the BAL and the TRP- condition.

Analysis showed a main effect for Treatment $(\mathrm{F}(1,18)=77.1 ; \mathrm{p}=0.000)$ and for Time $(\mathrm{F}(1,18)=66.3 ; \mathrm{p}=0.000)$, and an interaction between Treatment and Time $(\mathrm{F}(1,18)=210.9 ; \mathrm{p}=0.000)$ for the TRP $/ \Sigma \mathrm{LNAA}$ ratio. ATD lowered the ratio by $80 \%$. The mean ratio in the BAL condition was $0.10(\mathrm{SE}=0.004)$ at t0 and 0.13 $(\mathrm{SE}=0.008)$ at $\mathrm{t} 5$. The mean ratio in the TRP- condition was $0.10(\mathrm{SE}=0.003)$ at t0 and $0.02(\mathrm{SE}=0.003)$ at $\mathrm{t} 5$. No significant difference $(\mathrm{T}=0.0 ; \mathrm{p}=1.0)$ in the ratio was present at baseline between the BAL and the TRP- condition.

\section{Subjective measures}

Data from the POMS questionnaire was complete for 18 participants (10 BAL first, 8 TRP- first). No effect of ATD was found for depression, anger, vigor and tension. A significant interaction between Time, Treatment and Order $(\mathrm{F}(1,16)=7.2 ; \mathrm{p}=0.017)$ was found for fatigue. Participants who started in the BAL session were less fatigued after ATD than participants who started in the TRPcondition. Data from the physical complaints list was complete for all twenty participants. An interaction between Treatment and Order $(\mathrm{F}(1,18)=5.3 ; \mathrm{p}=0.034)$ was found. In the TRP- session participants reported more physical complaints when this was their first session.

\section{Performance}

Performance data are presented in Table 1 and were complete for all 20 participants (10 BAL first, 10 TRP- first).

1) Error rates. Firstly, an effect of Trial Type $(\mathrm{F}(1,18)=83.6 ; \mathrm{p}=0.000)$ was found: participants made more errors on IC trials (mean percentage incorrect $=7.5$ ) than on $\mathrm{CO}$ trials (mean percentage incorrect=3.8). Secondly, an interaction between Treatment and Order $(F(1,18)=7.0 ; p=0.017)$ was found. Post hoc pairedsample t-tests showed that when participants started in the BAL condition they made more errors $(\mathrm{t}=-2.5 ; \mathrm{p}=0.034)$ after ATD. When participants started in the TRP- condition no effect of ATD $(\mathrm{t}=1.0 ; \mathrm{p}=0.34)$ was found. Thirdly, an 
interaction between Treatment and Trial Type $(\mathrm{F}(1,18)=5.7 ; \mathrm{p}=0.029)$ was found. However, post hoc paired-sample t-tests did not show an effect of ATD on CO trials $(\mathrm{t}=0.3 ; \mathrm{p}=0.75)$ or IC trials $(\mathrm{t}=-1.6 ; \mathrm{p}=0.14)$.

2a) Mean RT on correct $C O$ versus correct IC trials. Firstly, an effect of Trial Type $(\mathrm{F}(1,18)=191.5 ; \mathrm{p}=0.000)$ was found: participants responded faster on correct Co trials (mean RT=318 ms) than on correct IC trials (mean RT=337 ms). Secondly, an interaction between Treatment and Order $(\mathrm{F}(1,18)=16.7 ; \mathrm{p}=0.001)$ was found: participants who started in the BAL condition (mean RT=335 ms) responded faster $(\mathrm{t}=4.7 ; \mathrm{p}=0.001)$ after ATD (mean $\mathrm{RT}=316 \mathrm{~ms})$, participants who started in the TRP- condition did not show a change $(\mathrm{t}=-1.7 ; \mathrm{p}=0.12)$ in RT after ATD (BAL mean RT=324 ms; TRP- mean RT=335 ms). Thirdly, an interaction between Treatment, Trial Type and $\operatorname{Order}(\mathrm{F}(1,18)=9.7 ; \mathrm{p}=0.006)$ was found. For participants who started in the BAL condition an effect of Treatment $(\mathrm{F}(1,9)=21.7$; $\mathrm{p}=0.001)$, Trial Type $(\mathrm{F}(1,9)=89.19 ; \mathrm{p}=0.000)$ and an interaction between Treatment and Trial Type $(\mathrm{F}(1,9)=6.2 ; \mathrm{p}=0.035)$ was found. The interaction shows

Table 1. Mean values with standard deviations for the different performance measures

\begin{tabular}{|c|c|c|c|c|}
\hline \multirow[b]{2}{*}{ Trial Type } & \multicolumn{2}{|c|}{ BAL first $(n=10)$} & \multicolumn{2}{|c|}{ TRP- first $(n=10)$} \\
\hline & BAL & TRP- & BAL & TRP- \\
\hline \multicolumn{5}{|c|}{ Error rate in $\%$} \\
\hline $\mathrm{CO}$ & $\underline{5.7(3.6)}$ & $\underline{7.6(4.4)}$ & $9.1(5.3)$ & $6.1(3.1)$ \\
\hline IC & $\underline{11.7(4.1)}$ & $\underline{15.6(3.7)}$ & $16.1(5.6)$ & $15.2(5.7)$ \\
\hline \multicolumn{5}{|c|}{ Mean reaction times in $\mathrm{ms}$} \\
\hline correct $\mathrm{CO}$ & $\underline{324(26)}$ & $\underline{308(19)}$ & $315(43)$ & $323(33)$ \\
\hline incorrect $\mathrm{CO}$ & $\underline{292(34)}$ & $\underline{264(24)}$ & $290(73)$ & $288(52)$ \\
\hline correct IC & $\underline{346(29)}$ & $\underline{323(23)}$ & $333(45)$ & 346 (33) \\
\hline incorrect IC & $\underline{293(31)}$ & $\underline{275(27)}$ & $293(42)$ & $303(50)$ \\
\hline \multicolumn{5}{|c|}{ Post-error slowing in $\mathrm{ms}$} \\
\hline Post-correct & $\underline{337(26)}$ & $\underline{317(20)}$ & $320(43)$ & $329(33)$ \\
\hline Post-error & $\underline{367(48)}$ & $\underline{326(16)}$ & $342(54)$ & 358 (38) \\
\hline \multicolumn{5}{|c|}{ Sequential congruency effect in ms } \\
\hline IC-CO & $22(8)$ & $16(2)$ & $21(15$ & $25(11)$ \\
\hline IC-IC & $-5(10)$ & $-11(2)$ & $-15(16)$ & $-15(9)$ \\
\hline
\end{tabular}

Data from the first session is printed in bold face. Underscore is used to indicate significant effects. $\mathrm{CO}=$ congruent trials; $\mathrm{IC}=$ incongruent trials; $\mathrm{BAL}=$ balanced session; TRP- = tryptophan depleted session; IC-CO = incongruent trial after a congruent trial; IC-IC; incongruent trial after an incongruent trial. 
that IC trials $(\mathrm{t}=5.1 ; \mathrm{p}=0.001)$ were more affected by ATD than CO trials $(\mathrm{t}=3.8$; $\mathrm{p}=0.004)$. For participants who started in the TRP- condition only an effect of Trial Type was found $(\mathrm{F}(1,9)=102.4 ; \mathrm{p}=0.000)$.

2b) Mean RT on incorrect CO versus incorrect IC trials. An interaction between Treatment and $\operatorname{Order}(\mathrm{F}(1,18)=7.3 ; \mathrm{p}=0.015)$ was found: participants who started in the BAL condition (mean $\mathrm{RT}=293 \mathrm{~ms}$ ) responded faster $(\mathrm{t}=2.9 ; \mathrm{p}=0.017$ ) after ATD (mean RT=270 ms), participants who started in the TRP- condition did not show a change $(\mathrm{t}=-0.7 ; \mathrm{p}=0.51)$ in $\mathrm{RT}$ after ATD (BAL mean $\mathrm{RT}=291 \mathrm{~ms}$; TRP- mean RT=296 ms).

2c) Mean RT on correct $C O$ versus incorrect $C O$ trials. Firstly, an effect of Trial Type was found $(\mathrm{F}(1,18)=39.9 ; \mathrm{p}=0.000)$ : participants reacted faster on incorrect (mean RT=284 ms) than on correct trials (mean RT=318 ms). Secondly, an interaction between Treatment and Order was found $(\mathrm{F}(1,18)=7.2 ; \mathrm{p}=0.015)$ : participants who started in the BAL condition (mean $\mathrm{RT}=309 \mathrm{~ms}$ ) responded faster $(\mathrm{t}=3.6 ; \mathrm{p}=0.006)$ after ATD (mean $\mathrm{RT}=286 \mathrm{~ms}$ ), participants who started in the TRP- condition did not show a change $(\mathrm{t}=-0.43$; $\mathrm{p}=0.68)$ in RT after ATD (BAL mean RT=303 ms; TRP- mean RT=306 ms).

2d) Mean RT on correct IC versus incorrect IC trials. Firstly, an effect of Trial Type was found $(\mathrm{F}(1,18)=174.1 ; \mathrm{p}=0.000)$ : participants reacted faster on incorrect (mean RT=291 ms) than on correct trials (mean RT=337 ms). Secondly, an interaction between Treatment and Order was found $(\mathrm{F}(1,18)=15.9 ; \mathrm{p}=0.001)$ : participants who started in the BAL condition (mean $\mathrm{RT}=320 \mathrm{~ms}$ ) responded faster ( $\mathrm{t}=4.1 ; \mathrm{p}=0.003$ ) after ATD (mean $\mathrm{RT}=299 \mathrm{~ms}$ ), participants who started in the TRP- condition did not show a change $(\mathrm{t}=-1.88$; $\mathrm{p}=0.09)$ in RT after ATD (BAL mean RT=313 ms; TRP- mean RT=325 ms).

3) Post-error slowing. Firstly, an effect of Trial Type $(\mathrm{F}(1,18)=29.6 ; \mathrm{p}=0.000)$ was found: participants responded slower on a correct trial after an error trial (mean RT=348) than on a correct trial after a correct trial (mean RT=326). Secondly, an interaction between Treatment and Order $(F(1,18)=17.7 ; p=0.001)$ was found: participants who started in the BAL condition (mean RT=352 ms) responded faster $(\mathrm{t}=3.9 ; \mathrm{p}=0.004)$ after ATD (mean $\mathrm{RT}=322 \mathrm{~ms}$ ), participants who started in the TRP- condition did not show a change $(\mathrm{t}=-1.91 ; \mathrm{p}=0.09)$ in RT after ATD (BAL mean RT=331 ms; TRP- mean RT=343 ms).

4) Spatial congruency effect. Statistical analysis revealed an effect of Trial Type $(\mathrm{F}(1,18)=100.5 ; \mathrm{p}=0.000)$ : participants reacted faster after IC trials (IC-IC $=$ 11.5) than after $\mathrm{CO}$ trials (IC-CO=21.0). 


\section{ERN}

Usable data was obtained for 14 participants (7 BAL first, 7 TRP- first). Figure 1 shows the ERPs for correct and incorrect responses in the BAL condition. No effect of ATD was found on the difference score $(F(1,12)=0.1 ; p=0.8)$ between the most positive peak between $-80 \mathrm{~ms}$ and $80 \mathrm{~ms}$ and the most negative peak between $0 \mathrm{~ms}$ and $200 \mathrm{~ms}$, which was $10.3(\mathrm{SE}=4.6) \mu \mathrm{V}$ in the $\mathrm{BAL}$ and $10.0(\mathrm{SE}=5.5) \mu \mathrm{V}$ in the TRP- condition. The mean ERN in the BAL and the TRP- session is presented in Figure 2. No interaction between Treatment and Order was found.

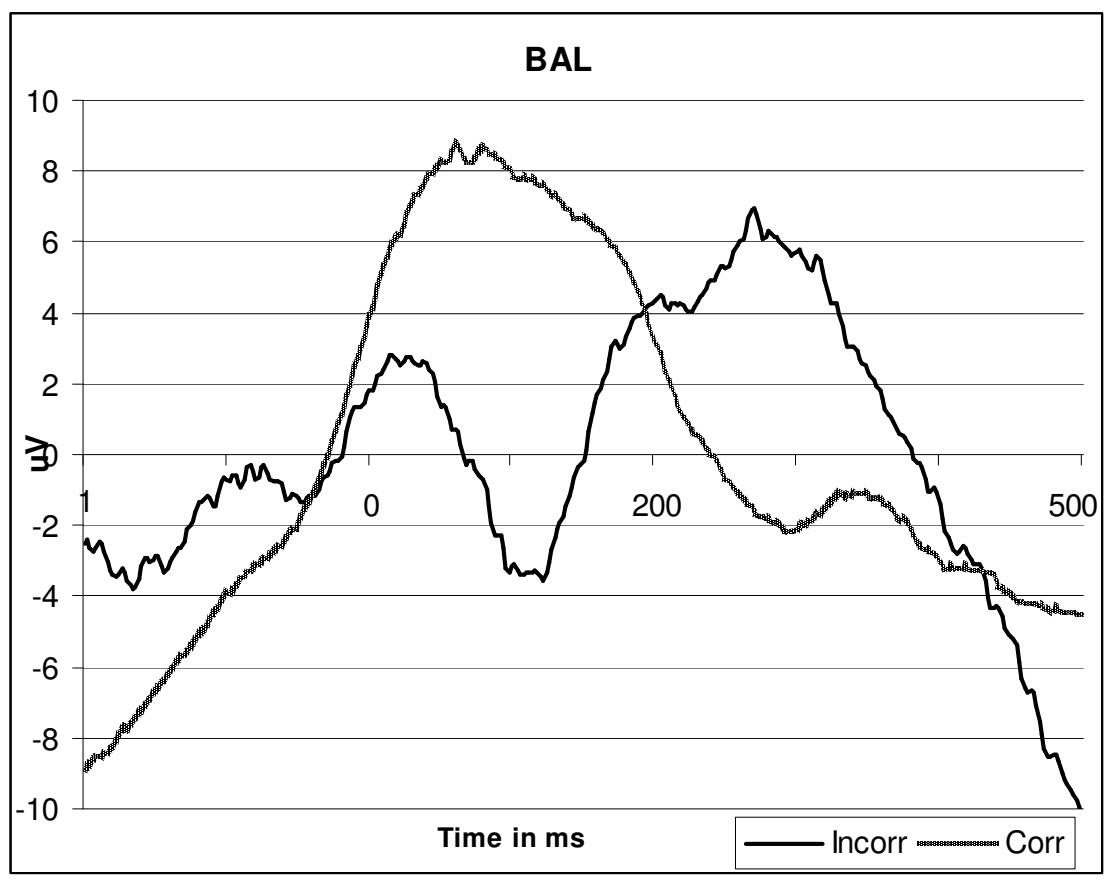

Figure 1. The weighted mean brain potential after a correct (Corr) and after an incorrect (Incorr) response in the balanced (BAL) condition is shown at the central electrode (CZ). 


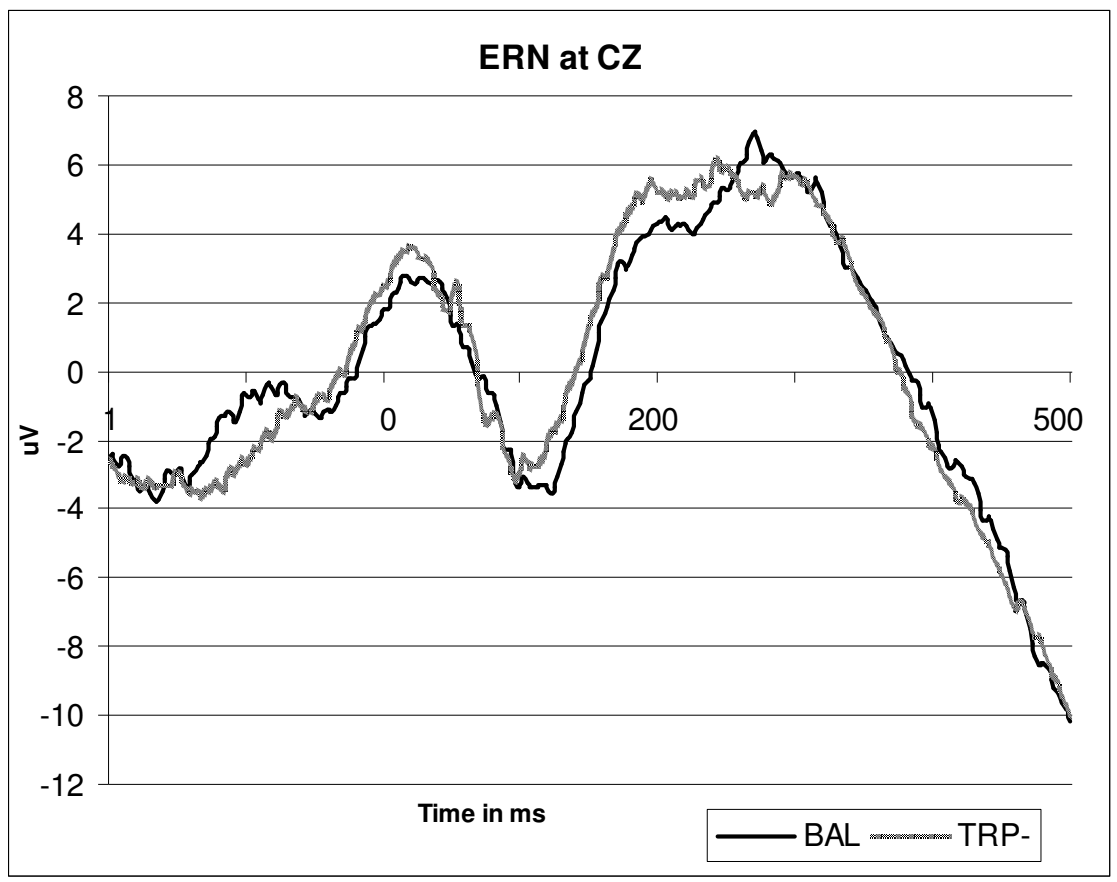

Figure 2. The weighted mean error-related negativity $(E R N)$ is shown in the balanced (BAL) and tryptophan depleted (TRP-) condition, at the central electrode (CZ).

\section{Discussion}

The present study investigated the effect of ATD on the ERN in an Eriksen Flanker Task. Performance was as expected: participants reacted faster 1) on CO than on IC trials, 2) after correct trials than after errors (post-error slowing) and 3) after IC than after CO trials (post congruency effect). During the BAL condition an ERN was found after a response error, which clearly differed from the positive ERP component after a correct response (see Figure 1). ATD did not affect the ERN amplitude, which is in agreement with studies that could not find effects of 5-HT manipulations on performance monitoring: De Bruijn et al. (2004; in press) showed that mirtazapine and paroxetine did not affect the ERN during an Eriksen Flanker Task.

Although no main effect of ATD was found, an interaction between Treatment and Order was found for most RT measures and the number of errors. When participants started in the BAL condition they responded faster and made more 
errors in the TRP- than in the BAL condition. However, when participants started in the TRP- condition no significant performance differences were found between the TRP- and the BAL condition. The mean RTs and percentage of errors presented in Table 2 suggest a learning effect for both group, however this effect seem be enhanced in the participants who started in the BAL condition. This finding is in accordance with studies that showed that ATD impaired learning and memory (e.g. Park et al., 1994; Schmitt et al., 2000; van der Veen et al., 2006).

The present study is in agreement with previous studies that did not show an effect of increased 5-HT levels (de Bruijn et al., 2004, in press) on the ERN. However, the current study seems to be in disagreement with studies that did suggest a role for 5-HT in performance monitoring (Evers et al., 2005, in press a; Fallgatter et al., 2005). In a previous study (Evers et al., in press a) we showed that ATD attenuated the activation in the dmPFC (BA 8) after a response error followed by negative feedback during a $\mathrm{Go} / \mathrm{NoGo}$ task. In this modified $\mathrm{Go} / \mathrm{NoGo}$ task we were not able to dissociate between the effect of ATD on error processing and the effect of ATD on negative feedback processing. The present study suggests that ATD does not affect error processing. We therefore hypothesize that ATD affects performance monitoring via a change in negative feedback processing, which is in line with the suggestion made in Evers et al. (2005). In that study we investigated the effect of ATD during a probabilistic reversal learning task. Four types of events were modeled: 1) correct responses followed by positive feedback, 2) correct responses followed by negative feedback (probabilistic errors), 3) incorrect responses where the participants reversed on the subsequent trial (reversal switch errors) and 4) incorrect responses where the participants did not reverse on the subsequent trial (reversal non-switch errors). The increased response in the dmPFC after ATD was significant when reversal switch errors were compared with correct responses, but was not when reversal switch errors were compared with reversal non-switch errors. This suggests that ATD does not affect behavioral switching during reversal learning. Because ATD marginally increased the BOLD signal during reversal non-switch errors relative to correct responses we hypothesized that ATD affects to sensitivity to negative feedback during reversal learning. This hypothesis needs to be tested in future research.

Furthermore, the results of the present study seem to be in contrast with the results of the study of Fallgatter et al. (2004). They reported that carriers of the short allele variant of a functional length variation in the transcriptional control region of the 5-HT transporter (5-HTT) gene showed higher amplitude of the ERN compared with carriers of two long alleles. Noteworthy, carriers of one or two short alleles of the 5-HTT gene, resulting in less 5-HT transport and therefore in lower 5-HT reuptake, presumably have higher synaptic 5-HT concentrations 
(analogue to 5-HTT knockout mice, for review see Hariri et al. 2006), whereas ATD reduces the availability of 5-HT. Another difference between the present and Fallgatters study (2004) is that in the present study 5-HT levels are manipulated only for a couple of hours while carriers of a short allele of the 5-HTT gene have chronically disturbed 5-HT levels. Interestingly, Ruchsow et al. (2004, 2006) showed that in depression, a disorder also related to chronically impaired 5-HT functioning (Risch and Nemeroff, 1992), the ERN is diminished during an Eriksen Flanker and a Go/NoGo task. These studies suggest that a transient lowering of 5HT does not affect the ERN, while chronically disturbed 5-HT functioning does change the ERN.

To conclude, in the present study we showed that a transient lowering of central 5-HT through ATD did not affect the ERN in healthy male volunteers. We hypothesize that ATD affects negative feedback processing but not error processing during performance monitoring. This hypotheses needs to be tested by future research. 


\section{Concluding remarks}

\section{The main results of this thesis}

\section{The main objective}

The main objective of the present thesis was to gain more insight into the role of the prefrontal cortex (PFC) and serotonin (5-HT) in cognitive flexibility in healthy volunteers. Cognitive flexibility refers to the ability to switch between behavioral strategies, when changes in the task lead to alterations in the outcome of the response. In a reversal learning task, for example, the learned stimulusresponse association will be changed during the task. Based on feedback, participants have to adjust their response to the new stimulus-response association when the reinforcement rule has changed. We showed that a transient lowering of central 5-HT increased the activation in the dorsomedial prefrontal cortex (dmPFC; Brodmann area (BA) 8) during a reversal switch in a reversal learning task (chapter 2) and decreased the activation in this area during performance monitoring in a Go/NoGo task (chapter 3). In addition, we showed that low 5-HT increased the activation in the anterior cingulate cortex (ACC; BA 32) during a Stroop task, when the task is not over learned (chapter 4). This increased activation was associated with improved performance during Stroop interference trials.

These studies suggest that low central 5-HT affects cognitive flexibility via activation changes in the dmPFC and the ACC. No effects of low 5-HT on brain activation were found during response inhibition (chapter 3). Likewise, no effects were found on the event-related negativity (ERN) (chapter 6).

\section{Interpretation of the results}

\section{The effect of ATD on cognitive flexibility}

In chapter 2 we showed that ATD increased the activation in the dmPFC during reversal learning. In the probabilistic reversal learning task used in this study four types of events were modeled: 1) correct responses followed by positive feedback, 2) correct responses followed by negative feedback (probabilistic errors), 3) incorrect responses where the participants reversed on the subsequent trial (reversal switch errors) and 4) incorrect responses where the participants did not reverse on the subsequent trial (reversal non-switch errors). The increased 
response in the dmPFC after ATD was significant when the reversal switch errors were compared with correct responses, but did not reach significance when the reversal switch errors were compared with the reversal non-switch errors. This suggests that ATD did not affect behavioral switching during reversal learning. Because ATD marginally increased the BOLD signal during the reversal nonswitch errors relative to correct responses, we hypothesized that ATD affects negative feedback processing during reversal learning. Depression has been related to low 5-HT functioning (Risch and Nemeroff, 1992). Previous studies reported that depressed patients showed a catastrophic response to failure (Beats et al., 1996) or oversensitivity to negative feedback (Elliot et al., 1997; Murphy et al., 2003). Based on these studies and our own results, we hypothesized that the increased BOLD signal in the dmPFC reflects enhanced processing of aversive signals (error feedback).

However, this study does not exclude the possibility that ATD affects response inhibition, error processing or response conflict during reversal learning. These possibilities were investigated in chapter 3 and 4 . In chapter 4 the effect of ATD on response inhibition and performance monitoring (brain activation after a response error followed by negative feedback) was examined. Because ATD did not affect response inhibition and the related brain activation, these results suggest that ATD does not influence cognitive flexibility by impairing response inhibition. On the other hand, this study revealed that ATD did change brain activation during performance monitoring: ATD decreased the activation in the dmPFC after a response error followed by negative feedback. This result is in line with our hypothesis that ATD changes processing of negative feedback.

In our last study (chapter 6 ) we investigated whether ATD affects the response to an error (ERN). Because the ERN (about $100 \mathrm{~ms}$ after response) is generated before negative feedback is given (about $600 \mathrm{~ms}$ after response), this study enabled us to isolate the effect of ATD on error processing from the effect of ATD on negative feedback processing. The results showed that ATD does not affect the ERN. Furthermore, in chapter 4 we showed that ATD improved performance during Stroop interference. This study suggests that ATD does not affect cognitive flexibility via a loss of cognitive control needed to overcome response interference.

To conclude, our studies suggest that ATD affects cognitive flexibility by changing the processing of negative feedback, rather than by impairing response inhibition, the response to an error or the loss of cognitive control.

\section{The dissociation between the effect of ATD on brain activation and performance}

In chapter 2 we showed that ATD increased the activation in the dmPFC during a reversal switch in a reversal learning task without affecting the number of 
reversals and the mean reaction time. Furthermore, in chapter 3 we showed that ATD decreased the activation in the dmPFC during performance monitoring in a $\mathrm{Go} /$ NoGo task without affecting the number of errors and the mean reaction time on a correct trial after a response error. We will discuss four possible explanations for the dissociation between the effect of ATD on brain activation and performance.

Firstly, the effect of ATD on performance might be hidden because of learning effects, as was suggested in chapter 2. In both studies the task was well practiced: the participants performed the task during a practice, a TRP- and a BAL session. Previous research showed that ATD impairs performance on a reversal learning task only on the first test session, in which the task was novel to the subjects (Park et al., 1994; Murphy et al., 2002).

Secondly, the cognitive tasks we used measured a combination of cognitive sub processes, while the present thesis suggests that not all of these processes are changed by ATD. For example, during a reversal switch in a reversal learning task the following sub processes are involved: performance monitoring, learning new stimulus-reward associations, inhibition of the response related to the previous stimulus-reward associations and behavioral adaptation. The present thesis suggests that ATD changes negative feedback processing which is a part of performance monitoring. Because performance monitoring is only one of the processes involved in a reversal switch it is possible that changes in negative feedback processing do not show in the behavioral outcome measures.

Thirdly, it is possible that ATD influences the cognitive strategy the participants used to successfully perform the tasks. Participants might need to recruit more or less effort, or participants might need to recruit other brain structures to reach the same level of performance. As a result brain activation patterns change, but performance is comparable. For example, Dibbets et al. (2006) showed that children with specific language impairment recruit other brain regions than children without this impairment during a task switching paradigm, while performance is comparable between these two groups.

Fourth and finally, neuroimaging might capture aspects of cognition that performance measures can not. Neuroimaging studies show brain activation patterns that underlie cognitive processes, while performance measures show the behavioral outcome of these brain mechanisms. Performance measures like reaction times and accuracy might therefore be imperfect measures of cognition. Our results might add to the suggestion that the BOLD response is more sensitive to detect changes in cognition than performance measures (Wilkinson and Halligan, 2004).

Importantly, the first three explanations for the dissociation between the effect of ATD on brain activation and performance support the fourth and final explanation: neuroimaging might capture aspects of cognition that performance 
might not. If the first explanation is correct, neuroimaging is able to show effects of ATD on reversal learning, while learning effects prevented effects of ATD on performance. If the second explanation is correct, neuroimaging is able to show effects of ATD on performance monitoring in a reversal learning and Go/NoGo task while performance measures were not able to dissociate between the effects of ATD on different cognitive sub processes. If the third explanation is correct, neuroimaging is able to show that ATD leads to brain activation changes due to changes in effort or cognitive strategy, while performance outcomes are unchanged. It remains to be tested by future research which of the first three explanations correctly explains the dissociation in our reversal learning and Go/NoGo study. Importantly, all of the given explanations show the importance of neuroimaging methods for cognitive research.

\section{The influence of biological and psychological factors}

It is possible that other factors that we did not control for have had an influence on the results. Studies that were not published at the time we started our experiments, but are available now, show that ATD modulates the amygdala activation in response to fearful faces as a function of self-reported threat sensitivity (Cools et al., 2005b). Interestingly, ATD impaired motivational guidance of goal-directed behavior on a cued-reinforcement reaction time task as a function of the trait impulsivity (Cools et al., 2005a). In addition, Roiser et al. (in press) showed that the effect of ATD on the vulnerability of loss of incentive motivation depends on the specific variant of the 5-HTT gene a person has.

In chapter 5, we confirmed the influence of biological and psychological factors on the effect of ATD: a family history of depression interacted with the effect of ATD on performance and brain activation during a Stroop task. Besides the Stroop task, the $\mathrm{FH}+$ and $\mathrm{FH}$ - participants were administered a facial recognition task (results reported in van der Veen, submitted) in a TRP- and a BAL session. This study showed that the mood response ATD induced in the FH+ group, leads to more interference from negative faces and a stronger amygdala response. As a part of chapter 3, we showed that the activation in the medial frontal gyrus (BA 6) positively correlated with the Barrett impulsivity score during response inhibition after ATD (chapter 3; unpublished data). This suggests that after ATD more activation is needed to withhold a response in impulsive participants. So, previous studies and our own results indicate the importance of individual difference in ATD research. These findings suggest that is it important for future research to control for biological and psychological factors that have shown to influence the effect of ATD.

This suggestion is supported by ATD studies that showed contradictory findings. For example, Rogers et al. (1999b) investigated the effect of ATD on a decision gambling task and showed that ATD decreased the tendency to choose 
the more likely outcome. Directly opposite are the results of Talbot et al. (2006). Talbot et al. used exactly the same decision gambling task and showed that ATD increased the tendency to choose the more likely outcome. Based on the very similar design and demographic characteristics of the studies, Talbot et al. suggests that it is possible that the different results are related to intrinsic trait characteristics of the participants tested. They therefore conclude that the contrast between these studies suggests the need for future ATD studies to control for personality, family history and genetic factors.

In conclusion, there are strong arguments for the execution of experiments in which the sources of individual variability are taken into consideration and even are the primary research question. Future studies should be devoted to this new topic.

\section{The effect of ATD in the dmPFC}

We showed that ATD increased the activation in the dmPFC during reversal learning (chapter 2), but decreased the activation in the dmPFC during performance monitoring in a Go/NoGo task (chapter 3). In the preceding paragraph we indicated the importance of individual differences in ATD research. Biological differences, such as a genetic disposition to depression, and psychological differences, such as threat sensitivity and impulsivity, interact with the effect of ATD. These different factors are defined within different research areas and therefore do not exclude each other. It is possible that the groups of participants tested in the reversal learning and in the Go/NoGo study did not have comparable personality characteristics. For example, the participants in the reversal learning study were paid about 225 euros, while the participants in the Go/NoGo study were paid 75 euros. It is therefore possible that the participants tested in the Go/NoGo study were more curious, impulsive or thrill seeking, while the participants tested in the reversal learning study more likely participated because they needed the money.

Furthermore, it is possible that the opposite effect of ATD on dmPFC activation is associated with task-related differences. As was suggested in chapter 3 , this opposite effect might be related to the different meaning of negative feedback within the reversal learning and Go/NoGo task. In the probabilistic reversal learning task negative feedback after a last reversal error signaled a response error due to a change of the stimulus-response rule, whereas in the Go/NoGo task negative feedback signaled a response error due to suboptimal performance.

Furthermore, because in both studies the BOLD response was locked to the onset of the response, the opposite effect might be related to the difference in awareness of the correctness of the response. In the reversal learning task, the participants were not sure whether the reinforcement rule had changed at the 
moment made they made the response, only after negative feedback they knew that the rule had changed. In the Go/NoGo task on the other hand, the participants may have been aware of the error at the moment they responded, that is before they received negative feedback.

Apart from a difference in awareness of the correctness of the response, this also indicates a difference in informative value of the negative feedback: in the reversal learning task the feedback is more informative. This suggests that the direction of the effect of ATD on dmPFC activation might depend on the informative value of the negative feedback. Interesting in this regard is a study by Murphy et al. (2003) that showed that depressed patients are impaired on a task in which non-informative negative feedback is given, but are unimpaired on a task in which highly informative negative feedback is given. Obviously, these hypotheses about the direction of the effect of ATD on dmPFC activation need to be tested by future research.

\section{Interactions of low 5-HT with other neurotransmitters}

Previous studies showed that ATD successfully reduced central 5-HT turnover (Carpenter et al., 1998; Moreno et al., 2000; Nishizawa et al., 1997; Williams et al., 1999; Young et al., 1985, 1999). More recent studies investigated the effect of ATD on the 5-HT system. Two recent PET studies showed that ATD did not affect the binding potential of the 5-HT transporter (Praschak-Rieder et al., 2005; Talbot et al., 2005) and the 5-HT 1A receptor (de Haes et al., 2002). A PET study by Yatham et al. (2001) showed that ATD decreased the binding potential of the 5HT2 receptor in various cortical regions. This suggests that ATD decreased the 5HT2 receptor density. Obviously, these effects of ATD need to be replicated by other studies and more research is needed to examine the effect of ATD on other receptor types and aspects of the 5-HT system.

In addition, 5-HT manipulations might interact with other neurotransmitter systems. 5-HT plays an inhibitory role and thereby lowers the actions of other neurotransmitters (Mogilnicka et al., 1977; Robbins 1997). Therefore it is possible that a decrease of central 5-HT levels leads to an increase in other neurotransmitter levels such as dopamine (DA). For example, it has been suggested that the improvement of focused attention during Stroop performance after ATD might be the result of increased DA (Schmitt et al., 2000; chapter 5). However, effects of ATD on the DA system have not been found so far (Hood et al., 2005). Young et al. (1989) showed that tyrosine and the catecholamine metabolites were unchanged after ATD in primates. Carpenter et al. (1998) showed that despite a rise in tyrosine, no change in the amount of the major DA metabolite HVA (homovanillic acid) was found after ATD in human cerebrospinal fluid (CSF). Lieben et al. (2004) examined the effects of ATD with a gelatin CP mixture on the levels of 5HT, 5-HIAA, DA and the DA metabolite DOPAC (3,4-dihydroxyphenylacatic 
acid) in the rat brain. They showed that after ATD the levels of 5-HT and 5-HIAA were significantly reduced in the striatum, the hippocampus and the cortex, whereas the levels of DA and DOPAC were unchanged in these areas.

In line with these results we showed that ATD did not change the ERN (chapter 6). Because it had been shown that the ERN is affected by DA manipulations (de Bruijn et al., 2004, in press; Zirnheld et al., 2004), this study suggests that DA levels are unchanged in dmPFC regions after ATD. The effect of ATD seems to be specific for 5-HT, and suggests therefore that the effect of ATD on activation of dmPFC regions associated with cognitive flexibility (chapter 2, 3 and 4) are not due to effects of ATD on DA levels.

Although these studies suggest that ATD does not change DA levels, previous studies showed that it is possible that ATD interacts with acetylcholine (ACh) levels (Ohue et al., 1991, 1993). Robbins (2005) reported that the effects of ATD somewhat resemble the effects of prefrontal cholinergic loss on a serial reversal learning task (Roberts et al., 1990, 1992). Lieben et al. (2005) showed that the acetylcholinesterase inhibitor metrifonate appeared to reverse the effects of ATD on recognition memory. To better understand the mechanisms that underlay the effect of ATD on cognition, research is needed to investigate the effect of ATD on other neurotransmitter levels.

\section{The prefrontal cortex}

The PFC is implicated in higher cognitive or executive functions such as adequate planning, judgment, decision-making, anticipation or reasoning, and monitoring external as well as internal states. Because of the nature of these tasks, it is thought that the PFC plays an important role in cognitive control, in the ability to orchestrate action in accordance with internal goal (Miller and Cohen, 2001).

The main gyri of the PFC roughly comprise three main anatomical divisions: the lateral gyri, the orbitofrontal gyri and the medial wall. The areas within the PFC are richly interconnected (Barbas 1995). In addition, the PFC is connected to almost every other part of the brain, which makes the PFC well suited to control many aspects of behavior (Groenewegen and Uylings, 2000).

A review by Ridderinkhof et al. (2004a) suggests that the medial PFC is important for evaluation or performance monitoring, while the lateral and orbitofrontal regions are more important for regulative functions of the implementation of appropriate adjustments. Another recent review of neuroimaging research (Amodio and Frith, 2006), focusing on the medial PFC (including the anterior cingulate cortex (ACC) and the orbitofrontal cortex), has revealed that the mPFC can be divided in three subdivisions: 1) the posterior region of the rostral mPFC (dorsal ACC and possible pre-supplementary motor areas), involved in representing and continuously updating the value of possible future action in order to regulate behavior, 2) the anterior regions of the rostral 
mPFC, involved in self-knowledge and mentalizing and 3) the orbitofrontal region of the $\mathrm{mPFC}$, involved in processing information concerning rewards and punishments. These researchers concluded that the mPFC is associated with the integration of complex representations and anticipated outcomes.

The present thesis showed that ATD affects the activation of the dmPFC (BA 8 ) during cognitive flexibility. The dmPFC (BA 8) is a pre-supplementary motor area, which receives projections from dorsolateral and medial visual areas, visuomotor regions of the intraparietal sulcus, multimodal sensory areas, the thalamus, the dorsolateral PFC and from limbic areas, such as the amygdala (Barbas 1988, 1990; Kolb and Whishaw, 1996; Morel et al., 2005). These connections suggest a role of the dmPFC in the control of motor action. Amodio and Frith (2006) suggested that the dmPFC, as a possible part of the rostral mPFC, might be involved in representing and continuously updating the value of possible future action in order to control and regulate behavior. In a study by Volz et al. (2005), a parametric analysis revealed that activation within the posterior frontomedial cortex, particularly within mesial BA 8 , increased with increasing decision uncertainty (see also Volz et al., 2003, 2004). They suggested that activation within BA 8 and BA 32/24 may distinguish decision conflict from response conflict.

These studies suggest that the dmPFC is activated during decision uncertainty and is involved in future action in order to regulate behavior. This suggestion fits our data. A response leading to a last reversal error during a reversal learning task and a response error in a Go/NoGo task are likely to be accompanied by decision uncertainty. In addition, in both situations future action has to be considered in order to receive positive feedback again on the next trial. This suggests that ATD influences cognitive flexibility by influencing the process of regulation of future action in order to control behavior. This suggested effect of ATD on regulation of future action might coincide with the suggested effect of ATD on the processing of negative feedback (see the paragraph 'The effect of ATD on cognitive flexibility' of this chapter). However, in chapter 3 (Go/NoGo task) and 7 (Eriksen Flanker task) we showed that ATD did not effect the reaction time on the trials after negative feedback is received. Possible explanations for the dissociation between the effect of ATD on brain activation and behavioral measures were presented in the paragraph 'Dissociation between brain activation and behavioral changes'.

\section{Conclusions}

The present thesis increased our insight into the role of 5-HT and the structures within the PFC in cognitive flexibility, by successfully combining behavioral studies in humans, biopsychological interventions and approaches on the domain 
of cognitive neuroscience and thereby. We showed that a transient lowering of central 5-HT influences the activation in the dmPFC during reversal learning and performance monitoring in a $\mathrm{Go} / \mathrm{NoGo}$ task in healthy volunteers. The present thesis suggests that ATD affects cognitive flexibility by changing the processing of negative feedback. Furthermore, it suggests that ATD does not impair cognitive flexibility by changing behavioral switching (reversal learning task), response inhibition (Go/NoGo task), error processing (ERN during Eriksen Flanker task) or cognitive control during response interference (Stroop task). Previous research suggests that the dmPFC (BA 8) is involved in representing and continuously updating the value of possible future action. Based on the suggested function of the dmPFC, our data suggest that ATD influences cognitive flexibility by influencing the process of regulation of future action, for which the processing of negative feedback is important.

Another important result of the present thesis is that a genetic predisposition to depression interacts with the effect of ATD on performance and brain activation during a Stroop task in healthy females. This indicates that biological and psychological variables are able to explain part of the within-group variance. Future research should evaluate the mechanisms underlying individual differences and control for variables that have been shown to interact with the effect of ATD on cognitive flexibility and related cognitive processes. 


\section{References}

Amodio D, Frith CD (2006). Meeting of minds: the medial frontal cortex and social cognition. Nat Rev Neurosci. Apr; 7(4): 268-77.

Anderson IM, Richell RA, Bradshaw CM (2003). The effects of acute tryptophan depletion on probabilistic choice. Journal of Psychopharmacology 17(1): 3-7.

Aron AR, Robbins TW, Poldrack RA (2004). Inhibition and the right inferior frontal cortex. Trends Cogn Sci 8: 170-177.

Aronson SC, Black JE, McDougle CJ, Scanley BE, Jatlow P, Kosten TR et al (1995). Serotonergic mechanisms of cocaine effects in humans. Psychopharmacology (Berl.) 119: 179-185.

Asahi SOY, Okada G, Yamawaki S, Yokota N (2004). Negative correlation between right prefrontal activity during response inhibition and impulsiveness: An fMRI study. Eur Arch Psychiatric Clin Neurosc. 254: 245-251.

Ballmaier M, Toga AW, Blanton RE, Sowell ER, Lavretsky H, Peterson J et al (2004). Anterior cingulate, gyrus rectus, and orbitofrontal abnormalities in elderly depressed patients: an MRIbased parcellation of the prefrontal cortex. Am J Psychiatry 161: 99-108.

Barbas H (1988). Anatomic organization of basoventral and mediodorsal visual recipient prefrontal regions in the rhesus monkey. J Comp Neurol. Oct 15; 276(3): 313-42.

Barbas H De Olmos J (1990). Projections from the amygdala to basoventral and mediodorsal prefrontal regions in the rhesus monkey. J Comp Neurol. 300(4): 549-71.

Barbas H (1995). Anatomic basis of cognitive-emotional interactions in the primate prefrontal cortex. Neurosci Biobehav Rev. 19(3): 499-510.

Barnes J, Costall B, Coughlan J, Domeney AM, Gerrard PA, Kelly ME et al (1990). The effects of ondansetron, a 5-HT3 receptor antagonist, on cognition in rodents and primates. Pharmacol Biochem Behav. 35(4): 955-62.

Baumgarten HG, Grozdanovic Z (1997). In: Baumgarten HG and Göthert M, editors. Serotonergic neurons and 5-HT receptors in the CNS . $2^{\text {nd }}$ print. Heidelberg New York: Springer Verlag.

Beats B, Sahakian BJ, Levy R (1996). Cognitive performance in tests sensitive to frontal dysfunction in the elderly depressed. Psychol Med. 26: 591-603.

Bechara A, Damasio H, Damasio, AD (2000). Emotion, decision making and the orbitofrontal cortex. Cerebral cortex 10: 295-307.

Beck AT. Depression. Clinical, Experimental and Theoretical Aspects. New York, NY. Harper \& Row; 1967.

Benkelfat C, Ellenbogen MA, Dean P, Palmour RM, Young SN (1994). Mood-lowering effect of tryptophan depletion. Enhanced susceptibility in young men at risk for major affective disorder. Arch Gen Psychiatry 51(9): 687-97.

Bermpohl F, Pascual-Leone A, Amedi A, Merabet LB, Fregni F, Gaab N et al (2006). Dissociable networks for the expectancy and perception of emotional stimuli in the human brain. Neuroimage Apr 1; 30(2): 588-600.

Biggio G, Fadda F, Fanni P, Tagliamonte A, Gessa G (1974). Rapid depletion of serum tryptophan, brain tryptophan, 5-HT and 5 hydroxyindolacetic by a tryptophan-free diet. Life Sci. 14: 13211329.

Booij L, Van der Does W, Benkelfat C, Bremner JD, Cowen PJ, Fava M et al (2002). Predictors of mood response to ATD. A reanalysis. Neuropsychopharmacology 27(5): 852-61. 
Booij L, Van der Does AJ, Haffmans PM, Riedel WJ, Fekkes D, Blom MJ (2005). The effects of high-dose and low-dose tryptophan depletion on mood and cognitive functions of remitted depressed patients. J Psychopharmacol. 19(3): 267-275.

Botvinick M, Nystrom LE, Fissell K, Carter CS, Cohen JD (1999). Conflict monitoring versus selection-for-action in anterior cingulate cortex. Nature 402(6758): 179-81.

Botvinick M, Cohen JD, Carter CS (2004). Conflict monitoring and anterior cingulate cortex: an update. Trends in Cognitive Sciences 8(12): 539- 546.

Bremner JD, Innis RB, Salomon RM, Staib LH, Ng CK, Miller HL et al (1997). Positron emission tomography measurement of cerebral metabolic correlates of tryptophan depletion-induced depressive relapse. Arch Gen Psychiatry 54 (4): 364-374.

Brett M, Anton J-L, Valabregue R, Poline J-B (2002). Region of interest analysis using an SPM toolbox [abstract]. Neuroimage 16: S497.

Buhot M, Martin S, Segu L (2000). Role of serotonin in memory impairment. Ann Med 32: 210-221.

Bush G, Whalen PJ, Rosen BR, Jenike MA, Mc Inerney SC, Rauch SL (1998). The counting Stroop: An interference and facilitation effects for functional neuroimaging. Validation study with functional MRI. Human Brain Mapping 6: 270-282.

Carpenter LL, Anderson GM, Pelton GH, Gudin JA, Kirwin PD, Price LH et al (1998). Tryptophan depletion during continuous CSF sampling in healthy human volunteers. Neuropsychopharmacology 19: 26-35.

Carter CS, Braver TS, Barch DM, Botvinick MM, Noll D, Cohen JD (1998). Anterior cingulate cortex, error detection, and the online monitoring of performance. Science 280: 747-749.

Cherek DR, Lane SD (2000). Fenfluramine effects on impulsivity in a sample of adults with and without history of conduct disorder. Psychopharmacology (Berl.) 152: 149-156.

Chudasama Y, Robbins TW (2003). Dissociable contributions of the orbitofrontal and infralimbic cortex to pavlovian autoshaping and discrimination reversal learning: further evidence for the functional heterogeneity of the rodent frontal cortex. J Neurosci. 23: 8771-8780.

Clark L, Cools R, Evers LE, van der Veen F, Jolles J, Sahakian BJ et al (2004). Neurochemical modulation of prefrontal cortex function. FENS Abstracts 2: A205.1.

Clark L, Roiser JP, Cools R, Rubinsztein DC, Sahakian BJ, Robbins TW (2005). Stop signal response inhibition is not modulated by tryptophan depletion or the serotonin transporter polymorphism in healthy volunteers: implications of the 5-HT theory of impulsivity. Psychopharmacology (Berl.) 182(4): 570-8.

Clarke HF, Dalley JW, Crofts HS, Robbins TW, Roberts AC (2004). Cognitive inflexibility after prefrontal serotonin depletion. Science 304: 878-880.

Coccaro EF, Siever LJ, Klar HM, Maurer G, Cochrane K, Cooper TB et al (1989). Serotonergic studies in patients with affective and personality disorders. Correlates with suicidal and impulsive aggressive behavior. Arch Gen Psychiatry 46: 587-599.

Compton RJ, Banich MT, Mohanty A, Milham MP, Herrington J, Miller GA et al (2003). Paying attention to emotion. An fMRI investigation of cognitive and emotional Stroop tasks. Cognitive, Affective, \& Behavioral Neuroscience 3(2): 81-96.

Cools R, Clark L, Owen AM, Robbins TW (2002). Defining the neural mechanisms of probabilistic reversal learning using event-related functional magnetic resonance imaging. The journal of neuroscience 22(11): 4563-4567.

Cools R, Calder AJ, Lawrence AD, Clark L, Bullmore E, Robbins TW (2005a). Individual differences in threat sensitivity predict serotonergic modulation of amygdala response to fearful faces. Psychopharmacology (Berl.) 180(4): 670-9. 
Cools R, Blackwell A, Clark L, Menzies L, Cox S, Robbins TW (2005b). Tryptophan depletion disrupts the motivational guidance of goal-directed behavior as a function of trait impulsivity. Neuropsychopharmacology 30 (7): 1362-1373.

Coull JT, Sahakian BJ, Middleton HC, Young AH, Park SB, McShane RH et al (1995). Differential effects of clonidine, haloperidol, diazepam and tryptophan depletion on focused attention and attentional search. Psychopharmacology (Berl.) 121: 222-230.

Crean J, Richards JB, de Wit H (2002). Effect of tryptophan depletion on impulsive behavior in men with or without a family of history of alcoholism. Behaviour Brain Research 136: 349-357.

Cusack R, Brett M, Osswald K (2003). An evaluation of the use of magnetic field maps to undistort echo-planar images. Neuroimage 18: 127-142.

Dalley JW, Theobald DE, Eagle DM, Passetti F, Robbins TW (2002). Deficits in impulse control associated with tonically-elevated serotonergic function in rat prefrontal cortex. Neuropsychopharmacology 26: 716-728.

Danjou P, Hamon M, Lacomblez L, Warot D (1990). Psychomotor, subjective and neuroendocrine effects of acute tryptophan depletion in the healthy volunteer. Psychiatry Psychobiol. 5: 3138.

De Bruijn E, Hulstijn W, Verkes RJ, Ruigt G SF, Sabbe BGC (2004). Drug-induced stimulation and suppression of action monitoring in healthy volunteers. Psychopharmacology (Berl.) 177: 151-160.

De Bruijn E, Sabbe BG, Hulstijn W, Ruigt GS, Verkes RJ (in press). Effects of antipsychotic and antidepressant drugs on action monitoring in healthy volunteers. Brain Res.

De Haes JI, Bosker FJ, Van Waarde A, Pruim J, Willemsen, ATM et al (2002). 5-HT1A receptor imaging in the human brain: effects of tryptophan depletion and infusion on [18F]MPPF binding. Synapse 46: 108-115.

Deakin JF (1991). Depression and 5HT. Int Clin Psychopharmacol. 6(Suppl 3): 23-28 disc. 29-31.

Dehaene S, Posner MI, Tucker DM (1994). Localization of a neural system for error detection and compensation. Psych Sci. 5: 303-305.

Dias R, Robbins TW, Roberts AC (1996). Dissociation in prefrontal cortex of affective and attentional shifts. Nature 380: 69-72.

Dibbets P, Bakker K, Jolles J (2006). Functional MRI of task switching in children with Specific Language Impairment (SLI). Neurocase 12(1): 71-79.

Drevets WC, Price JL, Simpson Jr JR, Todd RD, Reich T, Vannier M et al (1997). Subgenual prefrontal cortex abnormalities in mood disorders. Nature 386: 824-827.

Durston S, Thomas KM, Worden, MS, Yang Y, Casey BJ (2002). The effect of preceding context in inhibition: An event-related fMRI study. Neuroimage 16: 449-453.

Ellenbogen MA, Young SN, Dean P, Palmour RN, Benkelfat C (1996). Mood response to acute tryptophan depletion in healthy volunteers: Sex differences and temporal stability. Neuropsychopharmacology 15: 465-474.

Elliott R, Sahakian BJ, Herrod JJ, Robbins TW, Paykel ES (1996). Neuropsychological impairments in unipolar depression: the influence of perceived failure on subsequent performance. Psychol Med. 26: 975-989.

Elliott R, Sahakian BJ, Herrod JJ, Robbins TW, Paykel ES (1997). Abnormal response to negative feedback in unipolar depression: evidence for diagnosis specific impairment. Journal of Neurology, Neurosurgery and Psychiatry 63: 74-82.

Elliott R, Dolan RJ (1999). Ventromedial prefrontal cortex mediates guessing. Neuropsychologia 37: 403-411.

Elliott R, Rubinsztein JS, Sahakian BJ, Dolan RJ (2002). The neural basis of mood-congruent processing biases in depression. Arch Gen Psychiatry 59: 597-604. 
Eriksen B, Eriksen CW (1974). Effects of noise letters upon the identification of a target letter in a non-search task. Percept Psychophys. 16: 143-149.

Evenden JL (1999). Varieties of impulsivity. Psychopharmacology (Berl.) 146: 348-361.

Evers EA, Cools R, Clark L, van der Veen FM, Jolles J, Sahakian BJ, Robbins TW (2005). Serotonergic modulation of prefrontal cortex during negative feedback in probabilistic reversal learning. Neuropsychopharmacology 30(6): 1138-1147.

Evers EA, van der Veen FM, van Deursen JA, Schmitt JAJ, Deutz NEP, Jolles J (in press a). The effect of acute tryptophan depletion on the BOLD response during performance monitoring and response inhibition in healthy male volunteers. Psychopharmacology (Berl.)

Evers EAT, van der Veen FM, Jolles J, Deutz NEP, Schmitt JAJ (in press b). Acute tryptophan depletion improves performance and modulates the BOLD response during a Stroop task in healthy females. Neuroimage.

Fadda F (2000). Tryptophan-free diets: a physiological tool to study brain serotonin function. News Physiol Sci. 15: 260-264.

Fallgatter A, Merrmann MJ, Roemmler J, Ehlis AC, Wagener A, Heidrich A et al (2004). Allelic variation of serotonin transporter function modulates the brain electrical response for error processing. Neuropsychopharmacology 29(8): 1506-1511.

Fellows LK, Farah MJ (2003). Ventromedial frontal cortex mediates affective shifting in humans: evidence from a reversal learning paradigm. Brain 126: 1830-1837.

Fossati P, Ergis AM, Allilaire JF (2002). Executive functioning in unipolar depression: a review. Encephale 28(2): 97-107.

Fuster JM (1997). The prefrontal cortex, 3rd ed. New York: Raven Press.

Gallagher P, Massey AE, Young AH, McAllister-Williams RH (2003). Effects of acute tryptophan depletion on executive function in healthy volunteers. BMC Psychiatry 3: 10.

Garavan H, Ross TJ, Stein EA (1999). Right hemispheric dominance of inhibitory control: An eventrelated functional MRI study. PNAS 8301-8306.

Garavan H, Ross TJ, Murphy K, Roche RAP, Stein EA (2002). Dissociable executive functions in the dynamic control of behavior: inhibition, error detection, and correction. Neuroimage 17: $1820-1829$.

Garavan H, Ross TJ, Kaufman J, Stein EA (2003). A midline dissociation between error-processing and response-conflict monitoring. Neuroimage 20: 1132-1139.

Gartside SE, Cowen PJ, Sharp T (1992). Effect of amino-acid loads on hippocampal 5-HT release in vivo evoked by electrical stimulation of the dorsal raphe nucleus and D-fenfluramine administration. Br J Pharmacol. 107: 448P.

Gegenfurtner K, Kiper DC, (2003). Color vision. Annu Rev Neurosci. 26: 181-206.

Gehring WJ, Fencsik DE (2001). Functions of the medial frontal cortex in the processing of conflict and errors. J Neurosci. 21: 9430-9437.

Graeff FG, Brandao ML, Audi EA, Schutz MT (1986). Modulation of the brain aversive system by GABAergic and serotonergic mechanisms. Behav Brain Res. 22: 173-180.

Groenewegen H, Uylings HB (2000). The prefrontal cortex and the integration of sensory, limbic and autonomic information. Prog Brain Res. 126: 3-28.

Hariri AR, Drabant EM, Weinberger DR (2006). Imaging Genetics: Perspectives from Studies of Genetically Driven Variation in Serotonin Function and Corticolimbic Affective Processing. Biol Psychiatry May 15; 59(10): 888-97.

Harmer CJ, Rogers RD, Tunbridge E, Cowen PJ, Goodwin GM (2003). Tryptophan depletion decreases the recognition of fear in female volunteers. Psychopharmacology (Berl.) 167 (4): 411-7. 
Harrison AA, Everitt BJ, Robbins TW (1997). Central 5-HT depletion enhances impulsive responding without affecting the accuracy of attentional performance: interactions with dopaminergic mechanisms. Psychopharmacology (Berl.) Oct; 133(4): 329-42.

Harrison AA, Everitt BJ, Robbins TW (1999). Central serotonin depletion impairs both the acquisition and performance of a symmetrically reinforced go/no-go conditional visual discrimination. Behav Brain Res. Apr; 100 (1-2): 99-112.

Harrison BJ, Olver JS, Norman TR, Burrows GD, Wesnes KA, Nathan PJ (2004). Selective effects of acute serotonin and catecholamine depletion on memory in healthy women. $J$ Psychopharmacol. 18: 32-40.

Holroyd C, Coles M (2002). The neural basis of human error processing: reinforcement learning, dopamine, and the error-related negativity. Psychol Rev. 109(4): 679-709.

Hood S, Bell CJ, Nutt DJ (2005). Acute tryptophan depletion. Part I: rationale and methodology. Aust N Z J Psychiatry. 39(7): 558-64.

Horacek J, Zavesicka L, Tintera J, Dockery C, Platilova V, Kopecek M et al (2005). The effect of tryptophan depletion on brain activation measured by fMRI during the Stroop test in healthy volunteers. Physiol Res. 54(2): 235-44.

Horn N, Dolan M, Elliott R, Deakin JFW, Woodruff PWR (2003). Response inhibition and impulsivity: an fMRI study. Neuropsychologia 41: 1959-1966.

Hornak J, O'Doherty J, Bramham J, Rolls ET, Morris RG, Bullock PR et al (2004). Reward-related reversal learning after surgical excisions in orbito-frontal or dorsolateral prefrontal cortex in humans. J Cogn Neurosci. 16: 463-478.

Hornung J (2003). The human raphe nuclei and the serotonergic system. Journal of Chemical Neuroanatomy 26: 331-343.

Houston RJ, Bauer LO, Hesselbrock VM (2004). P300 evidence of cognitive inflexibility in female adolescents at risk for recurrent depression. Prog Neuropsychopharmacol Biol Psychiatry May; 28(3): 529-36.

Iversen SD, Mishkin M (1970). Perseverative interference in monkeys following selective lesions of the inferior prefrontal convexity. Exp Brain Res. 11: 376-386.

Jones B, Mishkin M (1972). Limbic lesions and the problem of stimulus-reinforcement associations. Exp Neurol 36: 362-377.

Kaiser S, Unger J, Kiefer M, Markela J, Mundt C, Weisbrod M (2003). Executive control deficit in depression: event-related potentials in a Go/NoGo task. Psychiatric Research: Neuroimaging 169: 169-184.

Kelly AMC, Hester R, Murphy K, Javitt DC, Foxe JJ, Garavan H (2004). Prefrontal-subcortical dissociation underlying inhibitory control revealed by event-related fMRI. European Journal of Neuroscience 19: 3105-3112.

Kerns JG, Cohen JD, MacDonald III AW, Cho RY, Stenger VA, Carter CS (2004). Anterior cingulate conflict monitoring and adjustments in control. Science 303: 1023-1026.

Klaassen T, Riedel WJ, van Someren A, Deutz NE, Honig A, van Praag HM (1999). Mood effects of 24-hour tryptophan depletion in healthy first-degree relatives of patients with affective disorders. Biol Psychiatry 15(46): 489-497.

Klaassen T, Riedel WJ, Deutz NE, Van Praag HM (2002). Mood congruent memory bias induces by tryptophan. Psychological Medicine 32(1): 167-172.

Kolb B, Wishaw IQ (1996). Fundamentals of human Neuropsychology. Fourth edition. W.H Freeman and company, Worth Publishers.

Koskinen T, Ruotsalainen S, Sirvio J (2000). The 5-HT(2) receptor activation enhances impulsive responding without increasing motor activity in rats. Pharmacol Biochem Behav. 66: 729738. 
Kringelbach ML, Rolls ET (2003). Neural correlates of rapid reversal learning in a simple model of human social interaction. Neuroimage 20: 1371-1383.

Lacerda AL, Keshavan MS, Hardan AY, Yorbik O, Brambilla P, Sassi RB et al (2004). Anatomic evaluation of the OFC in major depressive disorder. Biol Psychiatry 55: 353-358.

Laird A, McMillan KM, Lancaster JL, Kochunov P, Turkeltaub PE, Pardo JV, Fox PT (2005). A comparison of label-based review and ALE meta-analysis in the Stroop task. Human Brain Mapping 25(1): 6-21.

LeMarquand DG, Pihl RO, Young SN, Tremblay RE, Seguin JR, Palmour RM, Benkelfat C (1998). Tryptophan depletion, executive functions, and disinhibition in aggressive, adolescent males. Neuropsychopharmacology 19 (4): 333-341.

LeMarquand DG, Benkelfat C, Pihl RO, Palmour RM, Young SN (1999). Behavioral disinhibition induced by tryptophan depletion in nonalcoholic young men with multigenerational family histories of paternal alcoholism. Am J Psychiatry 156 (11): 1771-1779.

Leyton M, Okazawa H, Diksic M, Paris J, Rosa P, Mzengeza S et al (2001). Brain regional alpha[11C]methyl-L-tryptophan trapping in impulsive subjects with borderline personality disorder. Am J Psychiatry 158: 775-782.

Lezak MD (1995) Neuropsychological assessment. Oxford University, New York.

Liddle PF, Kiehl KA, Smith AM (2001). Event-related fMRI study of response inhibition. Human Brain Mapping 12: 100-109.

Lieben CK, Blokland A, Westerink B, Deutz NE (2004). Acute tryptophan and serotonin depletion using an optimized tryptophan-free protein-carbohydrate mixture in the adult rat. Neurochem Int. 44: 9-16.

Lieben C, Blokland A, Sik A, Sung E, van Nieuwenhuizen P, Schreiber R (2005). The selective 5HT6 receptor antagonist Ro4368554 restores memory performance in cholinergic and 5-HT models of memory deficiency in the rat. Neuropsychopharmacology 30(12): 2169-79.

Lim S, Kim JH (2005). Cognitive processing of emotional information in depression, panic, and somatoform disorder. J Abnorm Psychol. 114(1): 50-61.

Liu YP, Wilkinson LS, Robbins TW (2004). Effects of acute and chronic buspirone on impulsive choice and efflux of 5-HT and dopamine in hippocampus, nucleus accumbens and prefrontal cortex. Psychopharmacology (Berl.) 173: 175-185.

MacDonald AW 3rd, Cohen JD, Stenger VA, Carter CS (2000). Dissociating the role of the dorsolateral PFC and anterior cingulate cortex in cognitive control. Science 288: 1835-1838.

Maes M, Meltzer HY (1995). Psychopharmacology: the fourth generation of progress. Psychopharmacology: the serotonin hypothesis of major depression. Raven, New York.

Maldjian JA, Laurienti PJ, Kraft RA, Burdette JH (2003). An automated method for neuroanatomic and cytoarchitectonic atlas-based interrogation of fMRI data sets. Neuroimage 19: 1233-9.

Mandler, G., 1975. Mind and emotion, New York: Wiley.

Manes F, Sahakian B, Clark L, Rogers R, Antoun N, Aitken M, Robbins T (2002). Decision-making processes following damage to the prefrontal cortex. Brain 125(Pt 3): 624-39.

Marek GJ, Aghajanian GK (1998). 5-Hydroxytryptamine-induced excitatory postsynaptic currents in neocortical layer $\mathrm{V}$ pyramidal cells: suppression by mu-opiate receptor activation. Neuroscience 86: 485-497.

Matsumoto K, Tanaka K (2004). Conflict and cognitive control. Science 303: 969-970.

Mayberg HS, Liotti M, Brannan SK, McGinnis S, Mahurin RK, Jerabek PA et al (1999). Reciprocal limbic-cortical function and negative mood: converging PET findings in depression and normal sadness. Am J Psychiatry 156: 675-682.

Mazer C, Muneyyirci J, Taheny K, Raio N, Borella A, Whitaker-Azmitia P (1997). Serotonin depletion during synaptogenesis leads to decreased synaptic density and learning deficits in 
the adult rat: a possible model of neurodevelopmental disorders with cognitive deficits. Brain Res. 760(1-2): 68-73.

McKenna FP, Sharma D (1995). Intrusive cognitions: An investigation of the emotional Stroop task. Journal of experimental Psychology: Learning, memory \& cognition 21: 1595-1607.

McNair DM, Lorr DM, Droppelman LF (1988). Manual for the profile of mood states. San Diego, California.

Meneses A (1999). 5-HT system and cognition. Neurosci Biobehav Rev. 23: 1111-1125.

Menon V, Adleman NE, White CD, Glover GH, Reass AL (2001). Error-related brain activation during a Go/NoGo response inhibition task. Human Brain Mapping 12: 131-143.

Merali Z, Du L, Hrdina P, Palkovits M, Faludi G, Poulter MO et al (2004). Dysregulation in the suicide brain: mRNA expression of corticotropin-releasing hormone receptors and GABA(A) receptor subunits in frontal cortical brain region. J Neurosci 24: 1478-1485.

Miller E, Cohen JD (2001). An integrative theory of prefrontal cortex function. Annu Rev Neurosci. 24: 167-202.

Milner B (1964). Some effects of frontal lobectomy in man. In JM Warren and K Akert, eds. The frontal granular cortex and behaviour. New York: McGraw-Hill.

Mobini S, Chiang TJ, Ho MY, Bradshaw CM, Szabadi E (2000). Effects of central 5hydroxytryptamine depletion on sensitivity to delayed and probabilistic reinforcement. Psychopharmacology (Berl.) 152: 390-397.

Mogilnicka E, Scheel-Kruger J, Klimek V, Golembiowska-Nikitin K (1977). The influence of antiserotonergic agents on the action of DA drugs. Pol J Pharmacol Pharm. 29(1): 31-8.

Moja EA, Cipolla P, Castoldi D, Tofanetti O (1989). Dose-response decrease in plasma tryptophan and in brain tryptophan and serotonin after tryptophan-free amino acid mixtures in rats. Life Sci. 44: 971-976.

Moore P, Landolt HP, Seifritz E, Clark C, Bhatti T, Kelsoe J et al (2000). Clinical and physiological consequences of rapid tryptophan depletion. Neuropsychopharmacology 23: 601-622.

Morel A, Liu J, Wannier T, Jeanmonod D, Rouiller EM (2005). Divergence and convergence of thalamocortical projections to premotor and supplementary motor cortex: a multiple tracing study in the macaque monkey. Eur J Neurosci. Feb; 21(4): 1007-29.

Moreno F, McGavin C, Malan TP, Gelenberg AJ, Heninger GR, Mathe AA, Delgado PL (2000). Tryptophan depletion selectively reduces CSF 5-HT metabolites in healthy young men: results from single lumbar puncture sampling technique. Int J Neuropsychopharmacol. 3(4): 277-283.

Morris JS, Smith KA, Cowen PJ, Friston KJ, Dolan RJ (1999). Covariation of activity in habenula and dorsal raphe nuclei following tryptophan depletion. Neuroimage 10: 163-172.

Munafo M, Hayward G, Harmer C (2006). Selective processing of social threat cues following acute tryptophan depletion. J Psychopharmacol. 20(1): 33-9.

Munday J (2005). Instrumentation and electrode placement. Respir Care Clin N Am. 11(4): 605-15.

Murphy FC, Sahakian BJ, Rubinsztein JS, Michael A, Rogers RD, Robbins TW, Paykel ES (1999). Emotional bias and inhibitory control processes in mania and depression. Psychol Med. 29: 1307-1321.

Murphy FC, Smith KA, Cowen PJ, Robbins TW, Sahakian BJ (2002). The effects of tryptophan depletion on cognitive and affective processing in healthy volunteers. Psychopharmacology 163: 42-53.

Murphy FC, Michael A, Robbins TW, Sahakian BJ (2003). Neuropsychological impairment in patients with major depressive disorder: the effects of feedback on task performance. Psychological Medicine 33 (3): 455-467.

Neumeister A, Konstantinidis A, Stastny J, Schwarz MJ, Vitouch O, Willeit M et al (2002). Association between serotonin transporter gene promoter polymorphism (5HTTLPR) and 
behavioural responses to tryptophan depletion in healthy women with and without family history of depression. Arch Gen Psychiatry 59: 613-620.

Nishizawa S, Benkelfat C, Young SN, Leyton M, Mzengeza S, De Montigny C et al (1997). Differences between males and females in rates of serotonin synthesis in human brain. Proc. Nat. Acad. Sci. USA 94: 5308-5313.

Nunn J, Mathews A, Trower P (1997) Selective processing of concern-related information in depression. Br J Clin Psychol. 36(Pt 4): 489-503.

O'Doherty J, Kringelbach ML, Rolls ET, Hornak J, Andrews C (2001). Abstract reward and punishment representations in the human orbitofrontal cortex. Nature 4: 95-102.

Oatley K, Johnson-Laird PN (1987). Toward a cognitive theory of emotion. Cognition and emotion 1: $29-50$.

Ohue T, Koshimura K, Lee K, Watanabe Y, Miwa S (1991). A novel action of 6R-L-erythro-5,6,7,8tetrahydrobiopterin, a cofactor for hydroxylases of phenylalanine, tyrosine and tryptophan: enhancement of acetylcholine release in vivo in the rat hippocampus. Neurosci Lett. 128(1): 93-6.

Ohue T, Koshimura K, Takagi Y, Watanabe Y, Miwa S, Masaki T (1993). Enhancement of acetylcholine release in the hippocampus by $6 \mathrm{R}$-L-erythro-5,6,7,8-tetrahydrobiopterin is mediated by 5-hydroxytryptamine. Brain Res. 607(1-2): 255-60.

Orosco M, Rouch C, Beslot F, Feurte S, Regnault A, Dauge V (2004). Alpha-lactalbumin-enriched diets enhance serotonin release and induce anxiolytic and rewarding effects in the rat. Behav Brain Res. 148: 1-10.

Park SB, Coull JT, McShane RH, Young AH, Sahakian BJ, Robbins TW, Cowen PJ (1994). Tryptophan Depletion in Normal Volunteers Produces Selective Impairments in Learning and Memory. Neuropharmacology 33: 575-88.

Patkina NA, Lapin IP (1976). Effect of serotoninergic drugs on positive and negative reinforcing systems in cats. Pharmacol Biochem Behav. 5: 241-245.

Paus T, Petrides MJ, Evans AC, Meyer E (1993). Role of the human anterior cingulate cortex in the control of oculomotor, manual, and speech responses: a positron emission tomography study. J Neurophysiol. 70: 453-469.

Petrides M (1982). Motor conditional associative learning after selective prefrontal lesions in the monkey. Behavioural Brain research 5: 407-413.

Phan KL, Wager T, Taylor SF, Liberzon I (2002). Functional neuroanatomy of emotion: A metaanalysis of emotion activation studies in PET and fMRI. Neuroimage 16: 331-348.

Poeggel G, Nowicki L, Braun K (2003). Early social deprivation alters monoaminergic afferents in the orbital prefrontal cortex of Octodon degus. Neuroscience 116: 617-620.

Pollack I, Norman DA (1964). A non-parametric analysis of recognition experiments. Psychon Sci 1: $125-126$.

Praschak-Rieder N, Wilson AA, Hussey D, Carella A, Wei C, Ginovart N, Schwarz MJ, Zach J, Houle S, Meyer JH (2005). Effects of tryptophan depletion on the serotonin transporter in healthy humans. Biol Psychiatry 558(10): 825-30.

Preece MA, Dalley JW, Theobald DE, Robbins TW, Reynolds GP (2004). Region specific changes in forebrain 5-hydroxytryptamine1 A and 5-hydroxytryptamine2A receptors in isolation-reared rats: an in vitro autoradiography study. Neuroscience 123: 725-732.

Psychological Software Tools (2002). E-Prime. http://www.pstnet.com.

Puig MV, Artigas F, Celada P (2005). Modulation of the activity of pyramidal neurons in rat prefrontal cortex by raphe stimulation in vivo: involvement of serotonin and GABA. Cereb Cortex Jan; 15(1): 1-14.

Rabbit (1966). Errors and error correction in choice reaction time tasks. J Exp Psychol. 71: 264-272. 
Redgrave P, Horrell RI (1976). Potentiation of central reward by localised perfusion of acetylcholine and 5-hydroxytryptamine. Nature 262: 305-307.

Reilly JG, McTavish SF, Young AH (1997). Rapid depletion of plasma tryptophan: a review of studies and experimental methodology. J Psychopharmacol. 11: 381-392.

Remijnse P, Nielen MM, Uylings HB, Veltman DJ (2005). Neural correlates of a reversal learning task with an affectively neutral baseline: an event-related fMRI study. Neuroimage 26(2): 609618.

Ridderinkhof KR, van den Wildenberg WP, Segalowitz SJ, Carter CS (2004a). Neurocognitive mechanisms of cognitive control: The role of the PFC in action selection, response inhibition, performance monitoring, and reward-based learning. Brain and Cognition 56: 129-140.

Ridderinkhof RK, Ulsperger M, Crone EA, Nieuwenhuis S (2004b). The role of the medial frontal cortex in cognitive control. Science 306: 443-447.

Riedel WJ, Klaassen T, Deutz NEP, Someren van A, Praag van HM (1999). Tryptophan depletion in normal volunteers produces selective impairment in memory consolidation. Psychopharmacology 141: 362-369.

Riedel W, Klaassen T, Schmitt JA (2002). Tryptophan, mood, and cognitive function. Brain Behav Immun. 16(5): 581-9.

Riedel W, Sobczak S, Schmitt JA (2003). Tryptophan modulation and cognition. Adv Exp Med Biol. 527: 207-13.

Riedel W (2004). Cognitive changes after acute tryptophan depletion: what can they tell us? Psychol Med. 34: 3-8.

Rinck M, Becker ES (2005). A comparison of attentional biases and memory biases in women with social phobia and major depression. J Abnorm Psychol. 114(1): 62-74.

Risch S, Nemeroff CB (1992). Neurochemical alterations of serotonergic neuronal systems in depression. J Clin Psychiatry 53(Suppl): 3-7.

Robbins TW (1997). Arousal systems and attentional processes. Biological Psychology 45: 57-71.

Robbins TW (2000). Chemical neuromodulation of frontal-executive functions in humans and other animals. Exp Brain Res. 133: 130-138.

Robbins T (2005). Chemistry of the mind: neurochemical modulation of prefrontal cortical function. J Comp Neurol. 493(1): 140-6.

Roberts AC, Robbins TW, Everitt BJ, Jones GH, Sirkia TE, Wilkinson J, Page K (1990). The effects of excitotoxic lesions of the basal forebrain on the acquisition, retention and serial reversal of visual discriminations in marmosets. Neuroscience 34: 311-329.

Roberts AC, Robbins TW, Everitt BJ, Muir JL (1992). A specific form of cognitive rigidity following excitotoxic lesions of the basal forebrain in marmosets. Neuroscience 47: 251-264.

Rogers R, Blackhaw AJ, Middleton HC, Matthews K, Hawtin H, Crowley C et al (1999a). Tryptophan depletion impairs stimulus-reward learning while methylphenidate disrupts attentional control in healthy young adults: implications for the monoaminergic basis of impulsive behaviour. Psychopharmacology 146: 482-491.

Rogers R, Everitt BJ, Baldacchino A, Blackshaw AJ, Swainson R, Wynne K et al (1999b). Dissociable deficits in the decision-making cognition of chronic amphetamine abusers, opiate abusers, patients with focal damage to prefrontal cortex, and tryptophan-depleted normal volunteers. Neuropsychopharmacology 20(4): 322-339.

Rogers R, Tunbridge EM, Bhagwagar Z, Drevets WC, Sahakian BJ, Carter CS (2003). Tryptophan depletion alters decision-making of healthy volunteers through altered processing of reward cues. Neuropsychopharmacology 28(1): 153-162. 
Roiser J, Blackwell AD, Cools R, Clark L, Rubinsztein DC, Robbins TW, Sahakian BJ (in press). Serotonin Transporter Polymorphism Mediates Vulnerability to Loss of Incentive Motivation Following Acute Tryptophan Depletion. Neuropsychopharmacology.

Rosse RB, Schwartz BL, Zlotolow S, Banay-Schwartz M, Trinidad AC, Peace TD, Deutsch SI (1992). Effect of a low-tryptophan diet as an adjuvant to conventional neuroleptic therapy in schizophrenia. Clin Neuropharmacol 15(2): 129-41.

Rowley B, Van F, Mortimore C, Connell J (1997). Effects of acute tryptophan depletion on tests of frontal and temporal lobe function. J Psychopharmacol. (Oxford) 11: A60.

Rubia K, Smith AB, Brammer MJ, Taylor E (2003). Right inferior prefrontal cortex mediates response inhibition while mesial prefrontal cortex is responsible for error detection. Neuroimage 20: 351-358.

Rubia K, Lee F, Cleare AJ, Tunstall N, Fu CHY, Brammer M, McGuire P (2005). Tryptophan depletion reduces right inferior prefrontal activation during response inhibition in fast, eventrelated fMRI. Psychopharmacology (Berl.) 179 (4): 791-803.

Rubinsztein JS, Rogers RD, Riedel WJ, Mehta MA, Robbins TW, Sahakian BJ (2001). Acute tryptophan depletion impairs maintenance of "affective set" and delayed visual recognition in healthy volunteers. Springer, Berlin Heidelberg New York.

Ruchsow M, Herrnberger B, Beschoner P, Gron G, Spitzer M, Kiefer M (2006). Error processing in major depressive disorder: evidence from event-related potentials. J Psychiatr Res. 40(1): 3746.

Ruchsow M, Herrnberger B, Wiesend C, Gron G, Spitzer M, Kiefer M (2004). The effect of erroneous responses on response monitoring in patients with major depressive disorder: a study with event-related potentials. Psychophysiology 41(6): 833-40.

Sasaki-Adams DM, Kelley AE (2001). Serotonin-dopamine interactions in the control of conditioned reinforcement and motor behavior. Neuropsychopharmacology 25: 440-452.

Schmahmann JD, Caplan D (2006). Cognition, emotion and the cerebullum. Brain 129: 288-92.

Schmitt JA, Jorissen BL, Sobzak S, van Boxtel MP, Hogervorst E, Deutz NE, Riedel WJ (2000). Tryptophan depletion impairs memory consolidation but improves focused attention in healthy young volunteers. J Psychopharmacol. 14: 21-29.

Smith KA, Fairburn CG, Cowen PJ (1997). Relapse of depression after rapid depletion of tryptophan. Lancet 349: 915-919.

Smith KA, Morris JS, Friston KJ, Cowen PJ, Dolan RJ (1999). Brain mechanisms associated with depressive relapse and associated cognitive impairment following acute tryptophan depletion. British journal of psychiatry 174: 525-529.

Smith SM (2002). Fast robust automated brain extraction. Hum Brain Mapp. 17: 143-155.

Smith RL, Kennedy CH (2003). Increases in avoidance responding produced by REM sleep deprivation or serotonin depletion are reversed by administration of 5-hydroxytryptophan. Behav Brain Res. 140: 81-86.

Sobczak S, Riedel WJ, Booij L, Aan Het Rot M, Deutz NE, Honig A (2002). Cognition following acute tryptophan depletion: difference between first-degree relatives of bipolar disorder patients and matched healthy control volunteers. Psychol Med. 32(3): 503-515.

Soubrie P (1986). Reconciling the role of central serotonin neurons in human and animal behaviour. Behav Brain Res. 9: 319-364.

Steffens DC, Wagner HR, Levy RM, Horn KA, Krishnan KR (2001). Performance feedback deficit in geriatric depression. Biol Psychiatry 50: 358-363.

Swainson R, Rogers RD, Sahakian BJ, Summers BA, Polkey CE, Robbins TW (2000). Probabilistic learning and reversal deficits in patients with Parkinson's disease or frontal or temporal lobe lesions: possible adverse effects of dopaminergic medication. Neuropsychologia 38: 596-612. 
Talbot P, Frankle WG, Hwang D-R, Huang Y, Suckow RF, Slifstein M et al (2005). Effects of reduced endogenous 5-HT on in vivo binding of the serotonin transporter radioligand 11CDASB in healthy humans. Synapse 55: 164-175.

Talbot P, Watson DR, Barrett SL, Cooper SJ (2006). Rapid Tryptophan Depletion Improves Decision-Making Cognition in Healthy Humans without Affecting Reversal Learning or Set Shifting. Neuropsychopharmacology Jul; 31(7): 1519-25.

Talbot PS, Cooper SJ (in press). Anterior cingulate and subgenual prefrontal blood flow changes following tryptophan depletion in healthy males. Neuropsychopharmacology.

Teff KL, Young SN, Blundell JE (1989). The effect of protein or carbohydrate breakfasts on subsequent plasma amino acid levels, satiety and nutrient selection in normal males. Pharmacol Biochem Behav. 34: 829-837.

Tzourio-Mazoyer N, Landeau B, Papathanassiou D, Crivello F, Etard O, Delcroix N et al (2002). Automated anatomical labeling of activations in SPM using a macroscopic anatomical parcellation of the MNI MRI single-subject brain. Neuroimage 15: 273-289.

Van der Does AJW (2001). The effects of tryptophan depletion on mood and psychiatric symptoms. Journal of Affective Disorders 64: 107-119.

Van der Veen F, Evers EA, van Deursen JA, Deutz NE, Backes WH, Schmitt JA (2006). Acute tryptophan depletion reduces activation in the right hippocampus during encoding in an episodic memory task. Neuroimage Jul 1; 31(3): 1188-96

Van der Veen FM, Evers EAT, Deutz NEP, Schmitt JAJ (submitted). Effects of acute tryptophan depletion on mood and facial emotion perception related brain activation and performance in healthy women with and without a family history of depression.

Van Eijk HM, Rooyakkers DR, Deutz NE (1993). Rapid routine in amino acids in plasma by highperformance liquid chromatography with a 2-3 microns spherisorb ODS 11 column. $J$ Chromatogr. 620: 143-148.

Van Veen V, Carter CS (2002). The anterior cingulate as a conflict monitor: fMRI and ERP studies. Physiol Behav. 77(4-5): 477-82.

Veale D, Sahakian BJ, Owen AM, Marks IM (1996). Specific cognitive deficits in tests sensitive to frontal lobe dysfunction in obsessive-compulsive disorder. Psychol Med. 26(6): 1261-9.

Volz K, Schubotz RI, von Cramon DY (2003). Predicting events of varying probability: uncertainty investigated by fMRI. Neuroimage 19(2 Pt 1): 271-80.

Volz K, Schubotz RI, von Cramon DY (2005). Variants of uncertainty in decision making and their neural correlates. Brain Res Bull. 67(5): 403-412.

Volz KG, Schubotz RI, von Cramon DY (2004). Why am I unsure? Internal and external attributions of uncertainty dissociated by fMRI. Neuroimage Mar; 21(3): 848-57.

Wagner G, Sinsel E, Sobanski T, Köhler S, Maronou V, Mentzel H-J et al (2006). Cortical inefficiency in patients with unipolar depression: An event-related fMRI study with theStroop task. Biological Psychiatry 59(10): 958-65.

Walderhaug E, Lunde H, Nordvik JE, Landro NI, Refsum H, Magnusson A (2002). Lowering of serotonin by rapid tryptophan depletion increases impulsiveness in normal individuals. Psychopharmacology (Berl.) 164: 385-391.

Watson D, Clark LA, Tellegen A (1988). Development and validation of brief measures of positive and negative affect: the PANAS scales. J Pers Soc Psychol. 54: 1063-1070.

Weltzin TE, Fernstrom MH, Kaye WH (1994). 5-HT and bulimia nervosa. Nutr Rev. 52: 399-408.

Whalen P, Bush G, McNally RJ, Wilhelm S, McInerney SC, Jenike MA, Rauch SL (1998). Emotional counting Stroop paradigm: a functional magnetic resonance imaging probe of the anterior cingulate affective division. Biol Psychiatry 44(12): 1219-1228. 
Wilkinson LS, Humby T, Robbins TW, Everitt BJ (1995). Differential effects of forebrain 5hydroxytryptamine depletions on Pavlovian aversive conditioning to discrete and contextual stimuli in the rat. Eur J Neurosci 7: 2042-2052.

Wilkinson D, Halligan P (2004). The relevance of behavioural measures for functional-imaging studies of cognition. Neuroscience 5: 67-73.

Williams WA, Shoaf SE, Hommer D, Rawlings R, Linnoila M (1999). Effects of acute tryptophan depletion on plasma and cerebrospinal fluid tryptophan and 5-hydroxyindoleacetic acid in normal volunteers. J. Neurochem. 72: 1641-1647.

Winstanley CA, Theobald DEH, Dalley JW, Glennon JC, Robbins TW (2004). 5-HT2a and 5-HT2c receptor antagonists have opposing effects on a measure of impulsivity: interactions with global 5-HT depletion. Psychopharmacology (Berl.) 2004: 176 (3-4): 376-85.

Yatham L, Liddle PF, Shiah IS, Lam RW, Adam MJ, Zis AP, Ruth TJ (2001). Effects of rapid tryptophan depletion on brain 5-HT2 receptors: a PET study. British journal of psychiatry 178: 448-453.

Young S, Ervin FR, Pihl RO, Finn P (1989). Biochemical aspects of tryptophan depletion in primates. Psychopharmacology (Berl.) 98(4): 508-11.

Young S, Smith SE, Pihl RO, Ervin FR (1985). Tryptophan depletion causes a rapid lowering of mood in normal males. Psychopharmacology (Berl.) 87: 173-177.

Young SN, Leyton M, Benkelfat C (1999). Pet studies of serotonin synthesis in the human brain. Adv Exp Med Biol. 467 (11-18).

Young SN, Leyton M (2002). The role of serotonin in human mood and social interaction. Insight from altered tryptophan levels. Pharmacol Biochem Behav. 71(4): 857-65.

Zirnheld P, Carroll CA, Kieffaber PD, O'Donnell BF, Shekhar A, Hetrick WP (2004). Haloperidol impairs learning and error-related negativity in humans. J Cogn Neurosci. 16(6): 1098-112. 


\section{Summary}

In the introduction the aim and approach of this thesis are described and more detailed information about cognitive flexibility, serotonin (5-HT) and acute tryptophan depletion (ATD) is given. Previous research suggests that 5-HT is important for cognitive flexibility. Since the availability of functional Magnetic Resonance Imaging (fMRI) we are able to study which brain areas are activated during cognitive task performance. FMRI research showed the importance of structures within the prefrontal cortex (PFC) in higher cognitive functions. However, little is know about the effect of 5-HT manipulations on brain activation associated with cognition. The goal of the present thesis was to increase our insight into the role of different PFC structures and 5-HT in cognitive flexibility. We investigated the effect of ATD, a well recognized method to temporarily lower central 5-HT, on cognitive flexibility and the associated brain activation, in healthy volunteers.

In Chapter 1 we tested a novel method of ATD: a natural collagen protein (CP) mixture. Fifteen healthy volunteers were tested in a counterbalanced placebocontrolled within-subject design. Reversal learning, verbal memory and pattern recognition were assessed at baseline and 3-4 $\mathrm{h}$ after taking the $\mathrm{CP}$ mixture. The results show that this CP mixture successfully depleted plasma tryptophan (TRP) and the TRP/ $\Sigma$ LNAA (LNAA: large neutral amino acids) ratio. In the balanced (BAL) or placebo condition the CP mixture increased the TRP levels less drastically than the traditional amino acid (AA) mixture does. Delayed recognition reaction time on the verbal learning task was increased following ATD. No other effects were found. Possibly, the waiting period was too short to find effect of ATD on pattern recognition and reversal learning.

In Chapter 2 we investigated the effect of low 5-HT on brain activation associated with cognitive flexibility. In an event-related fMRI study we examined the effect of ATD on performance and the blood oxygen level dependent (BOLD) response during a probabilistic reversal learning task. Twelve healthy male volunteers were tested in a counterbalanced placebo-controlled within-subject design. The results show that ATD increased the activation in the dorsomedial prefrontal cortex (dmPFC) during a reversal switch. No effects of ATD on mood and performance were found. It was suggested that ATD changes the processing of negative feedback. 
Thereafter, we examined the effect of ATD on cognitive processes that are important for cognitive flexibility, such as response inhibition, performance monitoring and cognitive control that is needed to overcome response interference. In Chapter 3 an event-related fMRI study is described that examined the effect of ATD on response inhibition and performance monitoring during a modified Go/NoGo task. Thirteen healthy male volunteers were tested in a counterbalanced placebo-controlled within-subject design. The results show that ATD decreased the activation in the dmPFC during performance monitoring, but did not affect brain activation associated with response inhibition. No effects on mood and performance were found. In Chapter 4 an event-related fMRI study is presented that investigated the effect of ATD on performance and brain activation during a combined cognitive and emotional Stroop task. Fifteen healthy female volunteers were tested in a counterbalanced placebo-controlled within-subject design. The results show that ATD improved performance during interference trials. In addition, ATD increased the activation in the anterior cingulate cortex (ACC) during Stroop interference in the first Stroop block. These results suggest that the increased ACC activation triggered more cognitive control and thereby improved performance during interference trials.

Previous studies showed that ATD induced a mood response in participants with a predisposition to depression, but not in participants without such predisposition. In Chapter 5 we examined the effect of ATD on cognitive flexibility in healthy females with $(\mathrm{FH}+)$ and without $(\mathrm{FH}-)$ a family history of depression. In an event-related fMRI study we examined the effect of ATD on mood, cognitive performance and brain activation during a combined emotional and cognitive Stroop task. Twelve FH+ and fifteen FH- females were tested in a counterbalanced placebo-controlled within-subject design. ATD triggered a mood response in the FH+ group only. On the behavioral level, ATD decreased the Stroop interference score and accuracy in the FH- group, but increased accuracy in the FH+ group. Individual mood changes did not correlate with performance changes. However, individual mood changes correlated with activation in the ACC during Stroop interference: the more depressed after ATD, the more activation in the ACC. This study suggests that a genetic predisposition to depression interacts with the effect of ATD on mood and cognition.

To be able to isolate the effect of ATD on error processing from the effect of ATD on feedback processing, we investigated the effect of ATD on the errorrelated negativity (ERN): an event-related brain potential that appears before feedback is received and thought to originate from the ACC. In Chapter 6 an electroencephalogram (EEG) study is described that examined the effect of ATD on mood, cognitive performance and the ERN during an Eriksen Flanker task. 
Twenty healthy male volunteers were tested in a counterbalanced placebocontrolled within-subject design. On the behavioral level, ATD during the first session prevented faster responding on the second balanced session. With regard to the electrophysiological data, ATD did not change the amplitude of the ERN.

In the concluding remarks 1) the effect of ATD on cognitive flexibility, 2) the dissociation between the effect of ATD on brain activation and performance, 3) the influence of biological and psychological factors, 4) the effect of ATD in the dmPFC, 5) the interactions of low 5-HT with other neurotransmitters and 6) the prefrontal cortex. The most important finding of this thesis is firstly, that a transient lowering of central 5-HT changes the activation in the dmPFC during cognitive flexibility in healthy volunteers. The present thesis suggests that ATD affects cognitive flexibility by changing the processing of negative feedback. Furthermore, it suggests that ATD does not impair cognitive flexibility by affecting behavioral switching (reversal learning task), response inhibition (Go/NoGo task), error processing (ERN during Eriksen Flanker task) or cognitive control during response interference (Stroop task). Considering the involvement of the $\mathrm{dmPFC}$ in representing and updating the value of possible future action, our data suggests that ATD affects cognitive flexibility by influencing the process of regulation of future action. Secondly, this thesis showed that a genetic predisposition to depression interacts with the effect of ATD on performance and brain activation. Future research should evaluate the mechanisms underlying individual differences and control for variables that have shown to interact with the effect of ATD on cognitive flexibility. 


\section{Samenvatting}

Onderzoek toonde aan dat serotonine (5-HT) belangrijk is voor cognitieve flexibiliteit. Er is echter weinig bekend over het effect van 5-HT manipulaties op hersenactiviteit tijdens cognitieve taken. Sinds de ontwikkeling van functionele Magnetic Resonance Imaging (fMRI) zijn we in staat hersenactiviteit tijdens cognitieve taken te meten. FMRI onderzoek wees op het belang van prefrontale cortex (PFC) structuren voor hogere cognitieve functies. Het doel van dit proefschrift was om meer inzicht te verkrijgen in de rol van PFC structuren en 5HT in cognitieve flexibiliteit. We onderzochten het effect van acute tryptofaan depletie (ATD), een beproefde methode om 5-HT niveaus in het brein tijdelijk te verlagen, op cognitieve flexibiliteit en de daarmee samenhangende hersenactiviteit, in gezonde vrijwilligers. Naast het bespreken van de doelstelling van dit proefschrift en de gebruikte methoden, worden in de introductie cognitieve flexibiliteit, 5-HT en ATD nader besproken.

In hoofdstuk 1 testten we een nieuwe ATD methode: tryptofaan (TRP) depletie door middel van een natuurlijk collageen eiwitmengsel. Vijftien gezonde vrijwilligers werden getest in een gerandomiseerd placebogecontroleerd withinsubject design. Reversal leren, verbaal geheugen en patroonherkenning werden gemeten vóór en 3 tot 4 uur ná inname van het eiwitmengsel. Het eiwitmengsel verlaagde succesvol plasma TRP en de ratio tussen TRP en andere grote aminozuren. In de gebalanceerde of placeboconditie verhoogde het nieuwe mengsel TRP niveaus minder dan het traditionele aminozuurmengsel gewoonlijk doet. Na ATD nam de reactietijd tijdens woordherkenning toe. Er werden geen effecten van ATD op reversal learning en patroonherkenning gevonden. Dit is mogelijk te wijten aan een te korte periode tussen de inname van het eiwitmengsel en het testen.

In hoofdstuk 2 onderzochten we het effect van een verlaagd 5-HT niveau op hersenactiviteit tijdens cognitieve flexibiliteit. In een event-related fMRI studie onderzochten we het effect van ATD op prestatie en hersenactiviteit tijdens een probabilistic reversal learning taak. Twaalf gezonde mannelijke vrijwilligers werden getest in een gerandomiseerd placebo-controlled within-subject design. ATD verhoogde de activiteit in de dorsomediale PFC tijdens een reversal switch. Er werd geen effect van ATD op stemming en taakprestatie gevonden. De data suggereert dat ATD de verwerking van negatieve feedback beïnvloedt. 
Hierna onderzochten we het effect van ATD op cognitieve processen die belangrijk zijn voor cognitieve flexibiliteit, zoals response inhibitie, performance monitoring en cognitieve controle die nodig is tijdens response interferentie. In hoofdstuk 3 wordt een event-related fMRI studie beschreven waarin we het effect van ATD op prestatie en hersenactiviteit tijdens een aangepaste Go/NoGo taak onderzochten. Dertien gezonde mannelijke vrijwilligers werden getest in een gerandomiseerd placebogecontroleerd within-subject design. ATD verlaagde de activiteit in de dorsomedial PFC tijdens performance monitoring, maar had geen effect op hersenactiviteit tijdens response inhibitie. Er werden geen effecten gevonden op stemming en taakprestatie. In hoofdstuk 4 wordt een event-related fMRI studie gepresenteerd waarin we het effect van ATD op prestatie en hersenactiviteit tijdens een gecombineerde cognitieve en emotionele Stroop taak onderzochten. Vijftien gezonde vrouwelijke vrijwilligers werden getest in een gerandomiseerd placebogecontroleerd within-subject design. ATD verbeterde de prestatie tijdens Stroop interferentie en verhoogde de activiteit in de anterieure cingulate cortex (ACC) tijdens Stroop interferentie in het eerste Stroop blok. Deze resultaten suggereren dat de verhoogde ACC activatie meer cognitieve controle triggerde waardoor de prestatie verbeterde.

Onderzoek liet zien dat ATD stemming verlaagt in deelnemers met een genetische gevoeligheid voor depressie. In hoofdstuk 5 onderzochten we het effect van ATD op cognitieve flexibiliteit in gezonde vrouwen met $(\mathrm{FH}+)$ en zonder (FH-) depressie in de directe familie. In een event-related fMRI studie onderzochten we het effect van ATD op stemming, prestatie en hersenactiviteit tijdens een gecombineerde cognitieve en emotionele Stroop taak. Twaalf $\mathrm{FH}+$ en vijftien $\mathrm{FH}-$ vrijwilligers werden getest in een gerandomiseerd placebogecontroleerd within-subject design. ATD verlaagde de stemming in de FH+ groep. ATD verlaagde de Stroop interferentie score en de accuratesse in de $\mathrm{FH}-$, maar verhoogde de accuratesse in de FH+ groep. Individuele stemmingsveranderingen correleerden niet met taakprestatie maar wel met de activiteit in de ACC tijdens Stroop interferentie: hoe depressiever na ATD hoe meer activiteit in de ACC. Deze studie toont dat een genetische gevoeligheid voor depressie interacteert met het effect van ATD op stemming en cognitie.

Om het effect van ATD op het verwerken van fouten te kunnen scheiden van het effect van ATD op het verwerken van negatieve feedback, onderzochten we het effect van ATD op de 'error-related negativity' (ERN). In hoofdstuk 6 onderzochten we in een elektro-encefalografen (EEG) studie het effect van ATD op stemming, prestatie en de ERN tijdens een Eriksen Flanker taak. Twintig gezonde mannelijk vrijwilligers werden getest in een gerandomiseerd placebogecontroleerd within-subject design. ATD tijdens de eerste sessie 
voorkwam dat de proefpersonen sneller reageerden tijdens de tweede sessie. ATD had geen effect op de ERN.

We besluiten met een interpretatie van de resultaten. De volgende onderwerpen worden besproken: 1) het effect van ATD op cognitieve flexibiliteit, 2) de dissociatie tussen het effect van ATD op hersenactiviteit en gedrag, 3) de invloed van biologische en psychologische factoren, 4) het effect van ATD op activiteit in de dorsomediale PFC, 5) de interactie van verlaagd 5-HT met andere neurotransmitters en 6) de PFC. De belangrijkste bevinding van dit project is allereerst dat een tijdelijke verlaging van 5-HT de activatie in de dorsomediale PFC verandert terwijl gezonde vrijwilligers een taak uitvoeren die cognitieve flexibiliteit vereist. Dit proefschrift suggereert dat ATD cognitieve flexibiliteit verstoord via een verandering in de verwerking van negatieve feedback. Verder laat dit proefschrift zien dat ATD cognitieve flexibiliteit niet verstoort via een effect op gedragsaanpassingen (reversal learning taak), response inhibitie (Go/NoGo taak), het verwerken van fouten (performance monitoring tijdens Go/NoGo taak) of cognitieve controle tijdens response interferentie (Stroop taak). De dorsomedial PFC is betrokken bij het representeren en het updaten van de waarde van toekomstig gedrag. Dit proefschrift suggereert dan ook dat ATD cognitieve flexibiliteit beïnvloedt via de regulatie van toekomstig gedrag. Een tweede belangrijke bevinding is dat een genetische gevoeligheid voor depressie interacteert met het effect van ATD op stemming, gedrag en hersenactiviteit. Toekomstig onderzoek dient de mechanismen die ten grondslag liggen aan individuele verschillen te onderzoeken. Verder dient toekomstig ATD onderzoek te controleren voor variabelen waarvan bekend is dat ze interacteren met het effect van ATD op cognitieve flexibiliteit. 


\section{Curriculum vitae}

Lisbeth Evers werd geboren op 10 december 1972 te Heerlen. In 1991 behaalde zij haar VWO diploma aan het Stella Maris College in Meerssen. Van september 1997 tot juli 2002 studeerde zij psychologie aan de Universiteit Maastricht. Lisbeth studeerde af in de Biologische Psychologie. Na vier maanden als onderzoeksassistent gewerkt te hebben, deed ze van oktober 2002 tot september 2006 onderzoek als Assistent in Opleiding (AIO) bij het Instituut Hersenen en Gedrag, afdeling Psychiatrie en Neuropsychologie van de Universiteit Maastricht. Gedurende haar AIO periode voerde ze van maart tot juli 2003 een neuroimaging experiment uit in Cambridge (UK), in samenwerking met de afdeling Experimentele Psychologie van de Universiteit van Cambridge. Het onderzoek dat ze gedurende haar AIO periode deed staat beschreven in dit proefschrift.

\section{Publications}

Evers EAT, Tillie DE, van der Veen FM, Lieben CK, Jolles J, Deutz NEP, Schmitt JAJ (2005). Effects of a novel method of acute tryptophan depletion on plasma tryptophan and cognitive performance in healthy volunteers. Psychopharmacology (Berl.) Feb; 178(1): 92-9.

Evers EAT, Cools R, Clark L, van der Veen FM, Jolles J, Sahakian BJ, Robbins TW (2005). Serotonergic modulation of prefrontal cortex during negative feedback in probabilistic reversal learning. Neuropsychopharmacology Jun; 30(6): 1138-47.

Van der Veen FM, Evers EAT, van Deursen JA, Deutz NEP, Backes WH, Schmitt JAJ (2006). Acute tryptophan depletion reduces activation in the right hippocampus during encoding in an episodic memory task. Neuroimage Jul 1; 31(3): 1188-96.

Schmitt JAJ, Wingen M, Ramaekers JG, Evers EAT, Riedel WJ (2006). Serotonin and human cognitive performance. Current Pharmaceutical Design 12: 2473-2486.

Evers EAT, van der Veen FM, Jolles J, Deutz NEP, Schmitt JAJ (in press). Acute tryptophan depletion improves performance and modulates the BOLD response during a Stroop task in healthy females. Neuroimage.

Evers EAT, van der Veen FM, van Deursen JA. Schmitt JAJ, Deutz NEP, Jolles J (in press). The effect of acute tryptophan depletion on the BOLD response during performance monitoring and response inhibition in healthy male volunteers. Psychopharmacology (Berl.).

Van der Veen FM, Evers EAT, Deutz NEP, Schmitt JAJ (under revision). Effects of acute tryptophan depletion on mood and facial emotion perception related brain activation and performance in healthy women with and without a family history of depression. Neuropsychopharmacology. 
Van Nieuwenhoven MA, Kilkens TOC, Evers EAT, Brummer R-JM, WH Backes (submitted). Acute tryptophan depletion alters cortical activation following visceral stimuli. Gastroenterology.

Evers EAT, van der Veen FM, Jolles J, Deutz NEP, Schmitt JAJ (in preparation). A genetic predisposition to depression interacts with the effect of acute tryptophan depletion during a Stroop task.

Evers EAT, van der Veen FM, Deutz NEP, Vuurman EFPM, Jolles J (in preparation). The effects of acute tryptophan depletion on the error-related negativity; an EEG study. 